\title{
Complexity for charged thermofield double states
}

\author{
Mehregan Doroudiani, ${ }^{a, b}$ Ali Naseh ${ }^{a}$ and Reza Pirmoradian ${ }^{a, c}$ \\ ${ }^{a}$ School of Particles and Accelerators, Institute for Research in Fundamental Sciences (IPM), \\ P.O. Box 19395-5531, Tehran, Iran \\ ${ }^{b}$ Department of Physics, Sharif University of Technology, \\ P.O. Box 11365-9161, Tehran, Iran \\ ${ }^{c}$ Department of physics, Islamic Azad University Central Tehran Branch, \\ Tehran, Iran \\ E-mail: doroudiani@ipm.ir, naseh@ipm.ir, rezapirmoradian@ipm.ir
}

ABSTRACT: We study Nielsen's circuit complexity for a charged thermofield double state (cTFD) of free complex scalar quantum field theory in the presence of background electric field. We show that the ratio of the complexity of formation for cTFD state to the thermodynamic entropy is finite and it depends just on the temperature and chemical potential. Moreover, this ratio smoothly approaches the value for real scalar theory. We compare our field theory calculations with holographic complexity of charged black holes and confirm that the same cost function which is used for neutral case continues to work in presence of $\mathrm{U}(1)$ background field. For $t>0$, the complexity of cTFD state evolves in time and contrasts with holographic results, it saturates after a time of the order of inverse temperature. This discrepancy can be understood by the fact that holographic QFTs are actually strong interacting theories, not free ones.

KEYwORDS: AdS-CFT Correspondence, Black Holes, Gauge-gravity correspondence, Conformal Field Theory

ARXIV EPRINT: 1910.08806 


\section{Contents}

1 Introduction 1

2 cTFD states 4

3 Nielsen's complexity of cTFD state $\quad 9$

$\begin{array}{lll}3.1 & \text { Keeping the total size } L \text { of the system fixed } & 14\end{array}$

$\begin{array}{lll}3.2 & \text { Keeping the lattice spacing fixed with increasing } N & 15\end{array}$

4 Working with the infinite system $\quad 16$

4.1 Complexity of formation: A prob for prefer cost function 22

$\begin{array}{lll}4.2 & \text { Time dependency of complexity } 28\end{array}$

5 Conclusions $\quad 34$

$\begin{array}{ll}\text { A Thermal entropy and total charge } & 37\end{array}$

$\begin{array}{ll}\text { B Complexities in diagonal basis } & 37\end{array}$

C More concrete comparison with holography $\quad 40$

$\begin{array}{lll}\text { C.1 } & d=2+1 \text { dimensions } & 47\end{array}$

C.2 $d=3+1$ dimensions 48

\section{Introduction}

One of the most intriguing discoveries in the past decade is finding interesting connections between different quantum notions of information theory with quantum gravity and quantum field theory. These connections have been provided through Gauge/Gravity duality which the first one of them is reproducing entanglement entropy of conformal field theories (CFTs) using minimal co-dimension two surfaces in geometry of asymptotically locally AdS (AlAdS) spacetimes [1,2]. Intriguingly, this surface, which is known as the Ryu-Takayanagi surface, is also observed in a completely different area i.e. multi-scale entanglement renormalization ansatz (MERA) for a ground state of critical systems. It is observed by Swingle [3] that the Ryu-Takayanagi curve is similar to a special curve with minimum length in MERA tensor network and this similarity implies that gravity is an emergent quantity, indeed it emerges from entanglement entropy. Albeit this is a genuine idea, but it is discussed by Susskind [4] that entanglement entropy is not enough since it can not give information about the interior of black hole. This latter claim was a starting point to use another notion of quantum information theory in high energy physics community, i.e. computational complexity or more precisely state complexity. 
According to complexity action (CA) proposal of Susskind [5-7], the complexity of a boundary state is dual to a value of gravitational action in a special part of spacetime, known as WDW patch, which interestingly contains also the black hole interior. It is shown by Lloyd [8] that the complexity growth rate for a given state is smaller than twice the average energy of the system at that state and intriguingly the CA proposal gives the same value for neutral black holes at late times $[6,9,10]$. The ability of CA proposal to probe the interior of black holes not only gives us the opportunity to understand thermalization process in quantum many-body systems better, but also, by using that, one can hopefully resolve the black hole information paradox. Moreover, this proposal can play a great role in improving our understanding about scrambling and quantum chaos [11], ER=EPR [12] and firewalls $[13,14]$. It is worth noting that the main idea for this holographic proposal comes from rigorous computations for quantum mechanical systems. Accordingly, to understand the holographic results better, before anything, one needs to develop state complexity for QFTs with infinite degrees of freedom.

Let us remind that the complexity of a state $\left|\Psi_{\mathrm{T}}\right\rangle$ in a system of qubits is defined as the minimum number of gates which are needed to approximately produce target state from a reference state, $\left|\Psi_{R}\right\rangle$. The reference state is chosen as a state with no spatial entanglement entropy, i.e. ground state of a Hamiltonian with a very large mass in comparison with the kinetic term. Moreover, the gates are unitary operators and one calls the sequence of gates a circuit. Two main extensions of this idea have been recently made to quantify state complexity in QFTs. The first extension [15] is based on the idea of Nielsen [16-18] in which, one associates a geometry to the space of unitaries based on the algebra of gates. Then finding the optimal circuit is mapped to finding a minimal geodesic in the space of unitaries and the state complexity becomes the length of that geodesic. Up to now, this procedure has been well established for the free bosonic and fermionic QFTs [19-24] and preliminary steps for weakly interacting theories has been done in [25]. In the second extension, the optimal circuit is mapped to an optimized path integral definition of the target state [26, 27]. The intuition behind this extension comes from noting the relation between MERA and discretized path integral representation of a state. Accordingly, the cost of computing path integral on a Weyl-rescaled geometry is less than the cost of computing it on a flat twodimensional lattice. This cost is quantified by Liouville action and very interestingly it is shown [28] that this action has a deep connection with the length of minimal geodesics in the Nielsen approach i.e. in the first extension. Remarkably, both of the above extensions give rise to UV divergences similar to the ones in holographic computations. We explore the first extension in this paper and we discuss path integral complexity no more.

Recently, Chapman, Eisert, Hackl, Heller, Jefferson, Marrochio and Myers [29] evaluated the complexity of TFD state of a free scalar field theory. Their study led to discovery a cost function which is completely consistent with the properties of holographic complexity in the absence of any background charge. To discover these similarities, one special cost function, known as $F_{1}$ which will be explained below, is used for counting gates. By this cost function, the length of the path which connects reference state to the target state depends on the basis chosen for generators. Intriguingly, the advantage of this basis dependence has been taken in evaluating the complexity of formation and it is shown that it 
can produce the complexity of formation that compares well with holographic results. For U(1) charged black holes, a generalization of Lloyd's bound has been proposed [6],

$$
\frac{d \mathcal{C}_{A}}{d t} \leq \frac{2}{\pi}\left[(M-\mu Q)-(M-\mu Q)_{g s}\right]
$$

where the subscript $g s$ indicates a state which for a given chemical potential gives the lowest $(M-\mu Q)$. For charged small black holes, i.e. for the ones where the size of the horizon is much smaller than AdS radius, the rate of the change of complexity at late times saturates the bound

$$
\frac{d \mathcal{C}_{A}}{d t}=\frac{2}{\pi}(M-\mu Q)
$$

where for a given $\mu$, the smallest value of $(M-\mu Q)$ is zero. In this paper, we would like to extend the analysis of [29] to free complex scalar QFT in the presence of chemical potential. To be more precise, we would like to check whether that special cost function can also provide similar results with the holographic proposal (1.1), i.e., a linear growth of complexity at late times, its dependency on the detailed structure of null boundary terms, its UV divergence as spatial volume and its IR divergence for vanishing temperature at a fixed chemical potential. We will show that in contrast with holographic results, at late times, no linear growth appears in our calculation of complexity. It can be anticipated since the holographic results are applicable for strong interacting theories, but our results are obtained for free theory. Moreover, we see that, similar to the holographic proposal, the QFT result for complexity based on using $F_{1}$ cost function in Left-Right (LR) has IR divergences in the limit of zero temperature along with volume UV divergence. Furthermore, we discuss that by minor modification in the holographic proposal (adding a behind the horizon cut off), the detailed structure of null boundary terms can be encoded in the QFT results.

We organize the paper as follows: In section 2, we reconstruct the cTFD state of free complex scalar theory by putting it on a lattice with a UV cut off. In the same section, the time-dependent cTFD state is also studied. In section 3, after a brief review of covariance matrix formalism for manipulating the state complexity, we proceed to evaluate the complexity and its time dependency for cTFD state. In section 4 we extend the same analysis for continuous system and confirm that the prefer cost function introduced in [29] continues to work also in the presence of $\mathrm{U}(1)$ global charge. In appendix A some definitions are provided. Moreover, in appendix B the similar results for another basis are presented, i.e. diagonal one and in appendix $\mathrm{C}$ more concrete comparison with holographic complexity of charged black holes is provided.

Note added. When our paper was in the final stage to be submitted to arxiv, the paper [30] appeared which studied the same problem. The main difference between our approach with theirs is that we just have particles on the left side and as a result, just anti-particles on the right side. But their cTFD is made of particles and anti-particles on both sides, so their cTFD is a tensor product of our cTFD and a state with anti-particles on the left side and particles on the right. Our approach divides the phase space in half. Furthermore, it let us reach the conformal limit of the theory. 


\section{2 cTFD states}

In this section, we construct the cTFD state in the Hilbert space of free complex scalar theory. Just for simplicity we consider this theory in $(1+1)$ dimensions and in section 4 , we easily extend the study to general dimensions. The Lagrangian density of a free complex scalar field is given by

$$
\mathcal{L}=\left(D_{\mu} \Phi\right)\left(D_{\mu} \Phi\right)^{*}-m^{2} \Phi \Phi^{*},
$$

where the covariant derivative is defined by $D_{\mu}=\partial_{\mu}-i q A_{\mu}$. For the background electric field, $A_{\mu}=(-\mu, 0)$, so this Lagrangian density is simplified to

$$
\mathcal{L}=\dot{\Phi} \dot{\Phi}^{*}-\partial_{\vec{x}} \Phi \partial_{\vec{x}} \Phi^{*}-i \mu q\left(\dot{\Phi} \Phi^{*}-\Phi \dot{\Phi}^{*}\right)-\left(m^{2}-q^{2} \mu^{2}\right) \Phi \Phi^{*},
$$

with the dot means derivative with respect to the time. The conjugate momentums are given by

$$
\Pi=\frac{\partial \mathcal{L}}{\partial \dot{\Phi}}=\dot{\Phi}^{*}-i \mu q \Phi^{*}, \quad \Pi^{*}=\frac{\partial \mathcal{L}}{\partial \dot{\Phi}^{*}}=\dot{\Phi}+i \mu q \Phi,
$$

and the Hamiltonian on a circle with circumference $L$ becomes

$$
H=\int_{-\frac{L}{2}}^{\frac{L}{2}} d x\left(\Pi \Pi^{*}-i \mu q\left(\Pi \Phi-\Pi^{*} \Phi^{*}\right)+\partial_{x} \Phi \partial_{x} \Phi^{*}+m^{2} \Phi \Phi^{*}\right) .
$$

To regulate this theory in the UV, we consider that circle as a lattice with $N$ sites and lattice spacing $\delta$ which it implies that

$$
\delta=\frac{L}{N}, \quad \mathbf{x}_{a}=\Phi\left(x_{a}\right) \delta, \quad \mathbf{p}_{a}=\Pi\left(x_{a}\right),
$$

with $\mathbf{x}_{a}, \mathbf{p}_{a}$ are redefined canonical variables and $\mathbf{x}_{N+1}=\mathbf{x}_{1}$ and $\mathbf{p}_{N+1}=\mathbf{p}_{N}$. Following by these variables, the Hamiltonian (2.4) changes to

$$
H=\sum_{a=1}^{N}\left(\delta \mathbf{p}_{a} \mathbf{p}_{a}^{*}-i \mu q\left(\mathbf{p}_{a} \mathbf{x}_{a}-\mathbf{p}_{a}^{*} \mathbf{x}_{a}^{*}\right)+\frac{m^{2}}{\delta} \mathbf{x}_{a} \mathbf{x}_{a}^{*}+\frac{1}{\delta^{3}}\left(\mathbf{x}_{a+1}-\mathbf{x}_{a}\right)\left(\mathbf{x}_{a+1}^{*}-\mathbf{x}_{a}^{*}\right)\right) .
$$

By defining the following canonical transformation in momentum variable

$$
\mathbf{p}_{a}^{\prime}=\mathbf{p}_{a}+i \frac{\mu q}{\delta} \mathbf{x}_{a}^{*}
$$

the Hamiltonian (2.6) becomes

$$
H=\sum_{a=1}^{N}\left(\delta \mathbf{p}_{a}^{\prime} \mathbf{p}_{a}^{*}+\frac{m^{2}-\mu^{2} q^{2}}{\delta} \mathbf{x}_{a} \mathbf{x}_{a}^{*}+\frac{1}{\delta^{3}}\left(\mathbf{x}_{a+1}-\mathbf{x}_{a}\right)\left(\mathbf{x}_{a+1}^{*}-\mathbf{x}_{a}^{*}\right)\right)
$$

To decompose the contribution of different modes, one can use discrete Fourier transformation of variables as follows

$$
\tilde{\mathbf{x}}_{k}=\frac{1}{\sqrt{N}} \sum_{a=1}^{N} e^{\frac{2 \pi i k a}{N}} \mathbf{x}_{a}, \quad \tilde{\mathbf{p}}_{k}^{\prime}=\frac{1}{\sqrt{N}} \sum_{a=1}^{N} e^{\frac{-2 \pi i k a}{N}} \mathbf{p}_{a}
$$


where $\tilde{\mathbf{x}}_{k}^{*}=\tilde{\mathbf{x}}_{N-k}$ and similarly for the momentum variable. According to (2.9), the Hamiltonian (2.8) reduces to

$$
H=\sum_{k=0}^{N-1}\left(\delta\left|\tilde{\mathbf{p}}_{k}^{\prime}\right|^{2}+\frac{m^{2}-\mu^{2} q^{2}+\frac{4}{\delta^{2}} \sin ^{2}\left(\frac{\pi k}{N}\right)}{\delta}\left|\tilde{\mathbf{x}}_{k}\right|^{2}\right) .
$$

Now using the Fourier transformation $\tilde{\mathbf{p}}_{k}^{\prime}=\tilde{\mathbf{p}}_{k}+i \frac{\mu q}{\delta} \tilde{\mathbf{x}}_{N-k}^{*}$, the Hamiltonian (2.10) changes to

$$
H=\sum_{k=0}^{N-1}\left(\delta\left|\tilde{\mathbf{p}}_{k}\right|^{2}+\frac{\omega_{k}^{2}}{\delta}\left|\tilde{\mathbf{x}}_{k}\right|^{2}-i \mu q\left(\tilde{\mathbf{p}}_{k} \tilde{\mathbf{x}}_{N-k}-\tilde{\mathbf{p}}_{k}^{*} \tilde{\mathbf{x}}_{N-k}^{*}\right)\right),
$$

where $\omega_{k}$ is given by

$$
\omega_{k}^{2}=m^{2}+\frac{4}{\delta^{2}} \sin ^{2}\left(\frac{\pi k}{N}\right) .
$$

To construct the cTFD state of theory (2.11), one needs to quantize its Hamiltonian and it can be achieved by defining two sets of creation and annihilation operators

$$
\tilde{\mathbf{x}}_{k}=\sqrt{\frac{\delta}{2 \omega_{k}}}\left(\hat{a}_{N-k}+\hat{b}_{k}^{\dagger}\right), \quad \tilde{\mathbf{p}}_{k}=i \sqrt{\frac{\omega_{k}}{2 \delta}}\left(\hat{a}_{k}^{\dagger}-\hat{b}_{N-k}\right),
$$

with $\left[\hat{a}_{k}, \hat{a}_{k}^{\dagger}\right]=\left[\hat{b}_{k}, \hat{b}_{k}^{\dagger}\right]=1$ and other commutators are zero. Let remind that $\left(\hat{a}_{k}, \hat{a}_{k}^{\dagger}\right)$ and $\left(\hat{b}_{k}, \hat{b}_{k}^{\dagger}\right)$ are respectively the "annihilation, creation" operators for particles and antiparticles. Substituting (2.13) in (2.11) gives the following quantized Hamiltonian,

$$
\hat{H}=\sum_{k=0}^{N-1} \omega_{k}\left(\hat{a}_{k}^{\dagger} \hat{a}_{k}+\hat{b}_{k}^{\dagger} \hat{b}_{k}+1\right)+\mu q\left(\hat{a}_{k}^{\dagger} \hat{a}_{k}-\hat{b}_{k}^{\dagger} \hat{b}_{k}\right) .
$$

The Hamiltonian (2.14) contains two different parts. One part describes two standard harmonic oscillators for each mode (constant momentum $k$ )

$$
\hat{H}_{0}=\sum_{k=0}^{N-1} \omega_{k}\left(\hat{a}_{k}^{\dagger} \hat{a}_{k}+\hat{b}_{k}^{\dagger} \hat{b}_{k}+1\right) .
$$

By noting that the $\mathrm{U}(1)$ electric Noether charge associated to the complex scalar field reads as

$$
Q=q \int d x\left(\Phi^{*} \dot{\Phi}-\Phi \dot{\Phi}^{*}\right)
$$

the second part is the product of this charge operator with chemical potential

$$
\mu \hat{Q}=\sum_{k=0}^{N-1} \mu q\left(\hat{a}_{k}^{\dagger} \hat{a}_{k}-\hat{b}_{k}^{\dagger} \hat{b}_{k}\right)
$$

Accordingly, the Hamiltonian (2.11) constitutes $N$ decoupled harmonic oscillators with equal masses $M=\delta^{-1}$ (not to be confused with the physical mass $m$ ) and $k$-dependent frequencies $\omega_{k}$. If we omit the constant part, the total energy of level " $n$ " for each of these 
oscillators with fixed momentum $k$ is given by $n\left(\omega_{k}+\mu q\right)$. Since $\omega_{0}$ vanishes when $m=0$, the zero mode Hamiltonian does not have a normalizable ground state. But it can be regularized by introducing a very small dimensionless mass, $m L \ll 1$. Moreover, since each mode $k$ in (2.11) is decoupled from the other modes, the respective cTFD state will be the product of cTFD states for each oscillator. Accordingly, in the following, we focus on a single mode (fixed momentum $k$ ) and construct its time-dependent cTFD state. To attain that, let us remind that in thermal equilibrium, the ensemble average of any operator is given by

$$
\langle\hat{\mathcal{O}}\rangle_{\beta}=\frac{1}{Z} \operatorname{Tr}\left[e^{-\beta \hat{H}} \hat{\mathcal{O}}\right]=\frac{1}{Z} \sum_{n} e^{-\beta \mathcal{E}_{n, q}}\left\langle\mathcal{E}_{n, q}|\hat{\mathcal{O}}| \mathcal{E}_{n, q}\right\rangle
$$

where $Z$ is the partition function of the system and $\mathcal{E}_{n, q}=n(\omega+\mu q)$. One can express the above ensemble average of any operator as an expectation value in a thermal vacuum by defining a state $|0\rangle_{\beta}$ such that

$$
\langle\hat{\mathcal{O}}\rangle_{\beta}={ }_{\beta}\langle 0|\hat{\mathcal{O}}| 0\rangle_{\beta}=\frac{1}{Z} \sum_{n} e^{-\beta \mathcal{E}_{n, q}}\left\langle\mathcal{E}_{n, q}|\hat{\mathcal{O}}| \mathcal{E}_{n, q}\right\rangle
$$

Assuming thermal vaccum $|0\rangle_{\beta}$ can be written as a linear superposition of the states of Hilbert space $\left|\mathcal{E}_{n, q}\right\rangle$,

$$
|0\rangle_{\beta}=\sum_{n}\left|\mathcal{E}_{n, q}\right\rangle\left\langle\mathcal{E}_{n, q} \mid 0\right\rangle_{\beta}=\sum_{n} \alpha_{n, q}(\beta)\left|\mathcal{E}_{n, q}\right\rangle
$$

implies that

$$
{ }_{\beta}\langle 0|\hat{\mathcal{O}}| 0\rangle_{\beta}=\sum_{n, m} \alpha_{m, q}^{*}(\beta) \alpha_{n, q}(\beta)\left\langle\mathcal{E}_{m, q}|\hat{\mathcal{O}}| \mathcal{E}_{n, q}\right\rangle .
$$

This agrees with (2.19) provided

$$
\alpha_{m, q}^{*}(\beta) \alpha_{n, q}(\beta)=Z^{-1} e^{-\beta \mathcal{E}_{n, q}} \delta_{m n} .
$$

However, as it is clear from $(2.20), \alpha_{n, q}$ 's are ordinary numbers and therefore it is not possible to satisfy $(2.22)$. This means that we can not define the thermal vacuum as long as we restrict ourselves to the original Hilbert space. Interestingly, the condition (2.22) is quite analogous to orthonormality condition for state vectors. Let us introduce a fictitious system which is an identical copy of the original system and denote it with tilde system. Now, expanding the thermal vacuum as following

$$
|0\rangle_{\beta}=\sum_{n} \alpha_{n, q}(\beta)|n, \tilde{n}\rangle=\sum_{n} \alpha_{n, q}(\beta)\left|\mathcal{E}_{n, q}\right\rangle \otimes\left|\mathcal{E}_{\tilde{n},-q}\right\rangle
$$

implies that

$$
{ }_{\beta}\langle 0|\hat{\mathcal{O}}| 0\rangle_{\beta}=\sum_{n} \alpha_{n, q}^{*}(\beta) \alpha_{n, q}(\beta)\left\langle\mathcal{E}_{n, q}|\hat{\mathcal{O}}| \mathcal{E}_{n, q}\right\rangle .
$$

Comparing (2.24) with (2.19) implies that

$$
\alpha_{n, q}^{*}(\beta) \alpha_{n, q}(\beta)=Z^{-1} e^{-\beta \mathcal{E}_{n, q}},
$$


which has a simple real solution

$$
\alpha_{n, q}(\beta)=\alpha_{n, q}^{*}(\beta)=Z^{-1 / 2} e^{-\frac{\beta}{2} \mathcal{E}_{n, q}} .
$$

By Substituting $\alpha_{n, q}$ from (2.26) in (2.23), the charged thermal vacuum (cTFD state) becomes

$$
|\mathrm{cTFD}\rangle=Z^{-\frac{1}{2}} \sum_{n=0}^{\infty} e^{-\frac{\beta}{2} \mathcal{E}_{n, q}}\left|\mathcal{E}_{n, q}\right\rangle_{L} \otimes\left|\mathcal{E}_{n,-q}\right\rangle_{R},
$$

where we denoted the original system by subscript $R$ and the tilde system with subscript $L$, just in analogy with the right and left copies of CFT in the penrose diagram of eternal black hole. It is easy to see that the normalization factor becomes $Z^{-1}=1-e^{-\beta(\omega+\mu q)}$. To proceed furthermore, we note that the level " $n$ " energy eigenstate can be expressed as

$$
\left|\mathcal{E}_{n, q}\right\rangle=\frac{1}{\sqrt{n !}}\left(\hat{a}^{\dagger}\right)^{n}|0\rangle
$$

where

$$
\hat{a}^{\dagger}|n\rangle=\sqrt{n+1}|n+1\rangle, \quad \hat{a}|n\rangle=\sqrt{n}|n-1\rangle,
$$

with

$$
\hat{a}^{\dagger}=\sqrt{\frac{m \omega}{2}}\left(\hat{x}-i \frac{\hat{p}}{m \omega}\right), \quad \hat{a}=\sqrt{\frac{m \omega}{2}}\left(\hat{x}+i \frac{\hat{p}}{m \omega}\right),
$$

and $\left[\hat{a}, \hat{a}^{\dagger}\right]=1$. In comparison with (2.14) and noting to (2.15) and (2.17), it is clear that to construct the state $(2.27)$ one can use the $\left(\hat{a}_{k}, \hat{a}_{k}^{\dagger}\right)$ for the left side and $\left(\hat{b}_{k}, \hat{b}_{k}^{\dagger}\right)$ for the right side. This observation implies that $(2.27)$ can be simplified to

$$
|\mathrm{cTFD}\rangle=\sqrt{1-e^{-\beta(\omega+\mu q)}} \exp \left(e^{-\frac{\beta}{2}(\omega+\mu q)} \hat{a}_{L}^{\dagger} \hat{b}_{R}^{\dagger}\right)|0\rangle_{L} \otimes|0\rangle_{R}
$$

We would like to re-express the thermofield double state (2.31) by acting a unitary operator on the vacuum state $|0\rangle_{L} \otimes|0\rangle_{R}$. To achieve this, one can define the following operator

$$
\hat{\mathcal{O}}=\exp \left(\alpha_{+} \hat{\mathcal{K}}_{+}+\alpha_{-} \hat{\mathcal{K}}_{-}+\alpha_{0} \hat{\mathcal{K}}_{0}\right)
$$

with

$$
\hat{\mathcal{K}}_{-}=\hat{a}_{L} \hat{b}_{R} \quad \hat{\mathcal{K}}_{+}=\hat{a}_{L}^{\dagger} \hat{b}_{R}^{\dagger} \quad \hat{\mathcal{K}}_{0}=\frac{1}{2}\left(\hat{a}_{L}^{\dagger} \hat{a}_{L}+\hat{b}_{R}^{\dagger} \hat{b}_{R}+1\right),
$$

and

$$
\left[\hat{\mathcal{K}}_{-}, \hat{\mathcal{K}}_{+}\right]=2 \hat{\mathcal{K}}_{0}, \quad\left[\hat{\mathcal{K}}_{0}, \hat{\mathcal{K}}_{ \pm}\right]= \pm \hat{\mathcal{K}}_{ \pm}
$$

The operator $\hat{\mathcal{O}}$ is unitary for $\alpha_{+}=-\alpha_{-}$and $\alpha_{0} \in R$ and it can be decomposed as [31]

$$
\hat{\mathcal{O}}=e^{\gamma_{+} \hat{\mathcal{K}}_{+}} e^{\left(\log \gamma_{0}\right) \hat{\mathcal{K}}_{0}} e^{\gamma_{-} \hat{\mathcal{K}}_{-}}
$$

where

$$
\gamma_{0}=\left(\cosh \Theta-\frac{\alpha_{0}}{2 \Theta} \sinh \Theta\right)^{-2}, \quad \gamma_{ \pm}=\frac{2 \alpha_{ \pm} \sinh \Theta}{2 \Theta \cosh \Theta-\alpha_{0} \sinh \Theta}, \quad \Theta^{2}=\frac{\alpha_{0}^{2}}{4}-\alpha_{+} \alpha_{-} .
$$


For the special case $\alpha_{0}=0$ and $\alpha_{+}=-\alpha_{-}=\alpha \in \mathbb{R}$, the operator $\hat{\mathcal{O}}$ can be simplified to

$$
\hat{\mathcal{O}}=e^{\alpha\left(\hat{\mathcal{K}}_{+}-\hat{\mathcal{K}}_{-}\right)}=e^{(\tanh \alpha) \hat{\mathcal{K}}_{+}} e^{-2(\log \cosh \alpha) \hat{\mathcal{K}}_{0}} e^{-(\tanh \alpha) \hat{\mathcal{K}}_{-}} .
$$

By noting that

$$
\hat{\mathcal{K}}_{-}|0\rangle_{L} \otimes|0\rangle_{R}=0, \quad \hat{\mathcal{K}}_{0}|0\rangle_{L} \otimes|0\rangle_{R}=\frac{1}{2}|0\rangle_{L} \otimes|0\rangle_{R},
$$

we have

$$
e^{\alpha\left(\hat{\mathcal{K}}_{+}-\hat{\mathcal{K}}_{-}\right)}|0\rangle_{L} \otimes|0\rangle_{R}=\frac{1}{\cosh \alpha} e^{(\tanh \alpha) \hat{\mathcal{K}}_{+}}|0\rangle_{L} \otimes|0\rangle_{R}
$$

Now, by comparing the right hand side of (2.39) with (2.31), one can write the cTFD state (2.31) in desired form

$$
|\mathrm{cTFD}\rangle=e^{\alpha\left(\hat{a}_{L}^{\dagger} \hat{b}_{R}^{\dagger}-\hat{a}_{L} \hat{b}_{R}\right)}|0\rangle_{L} \otimes|0\rangle_{R} \quad \text { with } \quad \tanh \alpha=e^{-\frac{\beta}{2}(\omega+\mu q)} .
$$

The time evolution of state (2.40) is given by

$$
|\operatorname{cTFD}(t)\rangle=e^{-i\left(\hat{H}_{L}+\mu \hat{Q}_{L}\right) t_{L}} e^{-i\left(\hat{H}_{R}-\mu \hat{Q}_{R}\right) t_{R}}|\operatorname{cTFD}\rangle,
$$

with

$$
\hat{H}_{L}=\hat{a}_{L}^{\dagger} \hat{a}_{L}+\frac{1}{2}, \quad \hat{H}_{R}=\hat{b}_{R}^{\dagger} \hat{b}_{R}+\frac{1}{2}, \quad \mu \hat{Q}_{L}=\mu q \hat{a}_{L}^{\dagger} \hat{a}_{L}, \quad \mu \hat{Q}_{R}=-\mu q \hat{b}_{R}^{\dagger} \hat{b}_{R} .
$$

By choosing $t_{L}=t_{R}=t / 2$ following the common convention in holography, ${ }^{1}$ the timedependent cTFD state (2.41) after short computations becomes

$$
|\operatorname{cTFD}(t)\rangle=e^{-\frac{i}{2}(\omega+\mu q) t} \sqrt{1-e^{-\beta(\omega+\mu q)}} \exp \left[e^{-\frac{\beta}{2}(\omega+\mu q)} e^{-i(\omega+\mu q) t} a_{L}^{\dagger} b_{R}^{\dagger}\right]|0\rangle_{L} \otimes|0\rangle_{R} .
$$

To re-express the state (2.43) as acting a unitary operator on the $|0\rangle_{L} \otimes|0\rangle_{R}$, one can define

$$
z=\alpha e^{-i(\omega+\mu q) t}
$$

which by that and doing similar procedure as (2.32)-(2.39), this state becomes ${ }^{2}$

$$
|\operatorname{cTFD}(t)\rangle=\exp \left[z \hat{a}_{L}^{\dagger} \hat{b}_{R}^{\dagger}-z^{*} \hat{a}_{L} \hat{b}_{R}\right]|0\rangle_{L} \otimes|0\rangle_{R} .
$$

For future application, let us find the wave function representation of cTFD state (2.40). By defining the proper normal coordinates

$$
\hat{x}_{ \pm}=\frac{1}{\sqrt{2}}\left(\hat{x}_{L} \pm \hat{x}_{R}\right), \quad \hat{p}_{ \pm}=\frac{1}{\sqrt{2}}\left(\hat{p}_{L} \pm \hat{p}_{R}\right)
$$

\footnotetext{
${ }^{1}$ According to the boost symmetry, the evaluation of the holographic complexity will depend on $t=$ $t_{L}+t_{R}$ and not on each of the boundary times separately. So, without loss of generality, on can choose symmetric times $t_{L}=t_{R}=t / 2$.

${ }^{2}$ We have dropped the above global time-dependent phase, since this does not change the physical state.
} 
one can see

$$
\left(\hat{a}_{L}^{\dagger} \hat{b}_{R}^{\dagger}-\hat{a}_{L} \hat{b}_{R}\right)=-i\left(\hat{x}_{+} \hat{p}_{+}-\hat{x}_{-} \hat{p}_{-}\right),
$$

which this implies that (2.31) changes to

$$
|\mathrm{cTFD}\rangle=e^{-i \alpha\left(\hat{x}_{+} \hat{p}_{+}-\hat{x}_{-} \hat{p}_{-}\right)}|0\rangle_{+} \otimes|0\rangle_{-}=e^{-i \alpha \hat{x}_{+} \hat{p}_{+}}|0\rangle_{+} \otimes e^{i \alpha \hat{x}_{-} \hat{p}_{-}}|0\rangle_{-} .
$$

By noting that the ground-state wave functions are given by

$$
\langle x \mid 0\rangle_{ \pm} \equiv \Psi_{0}=\frac{(m \omega)^{\frac{1}{4}}}{\pi^{\frac{1}{4}}} e^{-\frac{m \omega}{2} x_{ \pm}^{2}},
$$

and

$$
\begin{aligned}
e^{-\frac{i \alpha}{2}\left(\hat{x}_{+} \hat{p}_{+}+\hat{p}_{+} \hat{x}_{+}\right)} \Psi_{0}\left(x_{+}\right) & \simeq \Psi_{0}\left(e^{-\alpha} x_{+}\right) \simeq e^{-\frac{m \omega}{2} e^{-2 \alpha} x_{+}^{2}} \\
e^{\frac{i \alpha}{2}\left(\hat{x}_{-} \hat{p}_{-}+\hat{p}_{-} \hat{x}_{-}\right)} \Psi_{0}\left(x_{-}\right) & \simeq \Psi_{0}\left(e^{\alpha} x_{-}\right) \simeq e^{-\frac{m \omega}{2} e^{2 \alpha} x_{-}^{2}},
\end{aligned}
$$

the wavefunctional cTFD state (2.48) becomes

$$
\left\langle x_{+}, x_{-} \mid \mathrm{cTFD}\right\rangle \equiv \Psi_{\mathrm{cTFD}} \simeq \exp \left[-\frac{m \omega}{2}\left(e^{-2 \alpha} x_{+}^{2}+e^{2 \alpha} x_{-}^{2}\right)\right] .
$$

In the standard coordinates $\left(x_{L}, x_{R}\right)(2.46)$, the above wave function changes to

$$
\Psi_{\mathrm{cTFD}} \simeq \exp \left[-\frac{m \omega}{2}\left(\cosh 2 \alpha\left(x_{L}^{2}+x_{R}^{2}\right)-2 \sinh 2 \alpha\left(x_{L} x_{R}\right)\right)\right] .
$$

Moreover, for later convenience let us write the time-dependent cTFD state (2.45), by using (2.47), as following

$$
|\operatorname{cTFD}(t)\rangle=e^{-i \alpha \hat{O}_{+}(t)}|0\rangle_{+} \otimes e^{i \alpha \hat{\mathcal{O}}_{-}(t)}|0\rangle_{-},
$$

with

$$
\hat{\mathcal{O}}_{ \pm}(t)=\frac{1}{2}\left(\cos ((\omega+\mu q) t)\left(\hat{x}_{ \pm} \hat{p}_{ \pm}+\hat{p}_{ \pm} \hat{x}_{ \pm}\right)+\sin ((\omega+\mu q) t)\left(m \omega \hat{x}_{ \pm}^{2}-\frac{1}{m \omega} \hat{p}_{ \pm}^{2}\right)\right) .
$$

By using this representation, one can easily find the time-dependent cTFD wavefunctional state similar to the procedure which is done in (2.48)-(2.52). Now, by having the cTFD state (and its time-dependent counterpart) one can calculate its complexity. The way we have chosen to calculate this quantity is based on the Nielsen geometric approach which is developed for QFTs in $[15,19]$. In the next section, we give a brief review on this approach and after that, we present the results for complexity with different cost functions.

\section{Nielsen's complexity of cTFD state}

As we discussed above, since each mode $k$ is decoupled from the other modes in (2.11), the respective cTFD state will be the product of TFD states for each of the oscillators. This means that the complexity of ground state of Hamiltonian (2.11) on a lattice with 
$N$ site is easily a sum over the complexity for each mode $k$. Therefore, it is sufficient to find the complexity for a single mode (2.51) and its time-dependent counterpart, (2.53), by using covariance matrix approach and then sum over all modes. To do that, let us provide firstly a brief review of Nielsen's geometric approach for evaluating circuit complexity. This geometric approach is a base for a group theoretic perspective to calculate the complexity of a state that is named as covariance matrix approach. As said before, the state complexity is defined as the minimal number of unitary gates required to prepare a certain target state $\left|\Psi_{T}\right\rangle$ from a specific reference state $\left|\Psi_{R}\right\rangle$, up to an error of $\epsilon$. The reference state $\left|\Psi_{R}\right\rangle$ is actually a factorizable state in position space which means that it is an unentangled state. Practically, the reference state is the ground state of the Hamiltonian

$$
H_{R}=\int_{-\frac{L}{2}}^{\frac{L}{2}} d x\left(\frac{1}{2} \Pi^{2}(x)+\frac{1}{2} \omega_{R}^{2} \Phi^{2}(x)\right)=\sum_{k=0}^{N-1}\left(\frac{\delta}{2}\left|\tilde{\mathbf{p}}_{k}\right|^{2}+\frac{\omega_{R}^{2}}{2 \delta}\left|\tilde{\mathbf{x}}_{k}\right|^{2}\right) .
$$

Nielsen and collaborators [16-18] introduced a geometric approach to identify the optimal unitary transformation $\hat{U}$ between reference and target state

$$
\left|\Psi_{T}\right\rangle=\hat{U}\left|\Psi_{R}\right\rangle
$$

as a string of continuous unitary operators

$$
\hat{U}=\mathcal{P} e^{-i \int_{0}^{1} d w \hat{H}(w)},
$$

where the path-dependent Hamiltonian $H(w)$ is expanded in terms of a basis of Hermitian operators $\hat{K}_{I}$ as following

$$
\hat{H}(w)=\sum_{I} Y^{I}(w) \hat{K}_{I}
$$

and $\mathcal{P}$ indicates the path ordering operator. It is worth noting that in the case of Gaussian states and with the mentioned gates, one does not need to consider a tolerance $\epsilon$, since the $Y_{I}(w)$ can always be adjusted to produce exactly the desired target state. Moreover, using this framework, one can consider trajectories in the space of unitaries

$$
\hat{U}(s)=\mathcal{P} e^{-i \int_{0}^{s} d w \hat{H}(w)},
$$

with the boundary conditions $\hat{U}(s=1)=\hat{U}, \hat{U}(s=0)=1$ and by that interprets $Y_{I}(s)$ as the tangent vector of the corresponding trajectory

$$
Y^{I}(s) \hat{\mathcal{O}}_{I}=\left(\partial_{s} \hat{U}(s)\right) \hat{U}^{-1}(s) .
$$

Now the state complexity is defined by the value of particular minimized cost $\mathcal{C}(U)$ defined by

$$
\mathcal{C}(U)=\int_{0}^{1} d s F(U(s), \vec{Y}(s))
$$

where the cost function $F(U(s), \vec{Y}(s))$ is a local functional along the trajectory of $U(s)$. This cost function has different forms, but the one which is the main focus in this paper is $[15]$

$$
F_{\kappa}(U, \vec{Y})=\sum_{I}\left|Y^{I}\right|^{\kappa}
$$


Intriguingly, in applying the above framework to a free scalar QFT in [15], a group theoretic structure was found to appear naturally. To see this structure, let us consider a bosonic system with $N$ degrees of freedom. This system can be described by $2 N$ observables $\hat{\xi}=$ $\left(\hat{q}_{1}, \hat{q}_{2}, \ldots, \hat{q}_{N}, \hat{p}_{1}, \ldots, \hat{p}_{N}\right)$ with $\left(\hat{q}_{i}, \hat{p}_{i}\right)$ are canonical operators. The two-point functions of these observables in an arbitrary state $|\Psi\rangle$ can be expressed as

$$
\left\langle\Psi\left|\hat{\xi}^{a} \hat{\xi}^{b}\right| \Psi\right\rangle=\frac{1}{2}\left(G^{a b}+i \Omega^{a b}\right)
$$

where $G^{a b}=G^{(a b)}$ is the symmetric part of the correlation matrix and $\Omega^{a b}=\Omega^{[a b]}$ denotes the antisymmetric part. In fact, for a system with bosonic degrees of freedom, $\Omega^{a b}$ is trivial and simply contains the information about commutation relations of $\hat{q}_{i}$ and $\hat{p}_{i}$. Restricting to the space of Gaussian states implies that the unitary operator $\hat{U}(s)$ can be expressed by Hermitian operators that are quadratic in the canonical operators $\hat{\xi}$,

$$
\hat{U}(s)=e^{-i s \hat{K}}, \quad \text { with } \quad \hat{K}=\frac{1}{2} \hat{\xi}^{a} k_{(a, b)} \hat{\xi}^{b} \equiv \frac{1}{2} \hat{\xi} k \hat{\xi}^{T} .
$$

To proceed further, one needs the operation of $\hat{U}(s)$ on $\hat{\xi}^{a}$ which can be obtained as follows

$$
\hat{U}^{\dagger}(s) \hat{\xi}^{a} \hat{U}(s)=\sum_{n=0}^{\infty} \frac{s^{n}}{n !}\left[i \hat{K}, \hat{\xi}^{a}\right]_{(n)},
$$

with $\left[i \hat{K}, \hat{\xi}^{a}\right]_{(n)}$ is defined recursively by $\left[i \hat{K}, \hat{\xi}^{a}\right]_{(n)}=\left[i \hat{K},\left[i \hat{K}, \hat{\xi}^{a}\right]_{(n-1)}\right]$, and $\left[i \hat{K}, \hat{\xi}^{a}\right]_{(0)}=$ $\left[i \hat{K}, \hat{\xi}^{a}\right]$. Using (3.10) and the commutation relation $\left[\hat{\xi}^{a}, \hat{\xi}^{b}\right]=i \Omega^{a b}$, it is easy to see that

$$
\left[i \hat{K}, \hat{\xi}^{a}\right]=\Omega^{a b} k_{(b, c)} \hat{\xi}^{c}
$$

which by defining $K_{b}^{a}=\Omega^{a c} k_{(c, b)}$, it can be written as

$$
\left[i \hat{K}, \hat{\xi}^{a}\right]=K_{b}^{a} \hat{\xi}^{b} .
$$

This latter identity implies that

$$
\hat{U}^{\dagger}(s) \hat{\xi}^{a} \hat{U}(s)=\left(e^{s K}\right)_{b}^{a} \hat{\xi}^{b} \equiv U(s)_{b}^{a} \hat{\xi}^{b} .
$$

Now, (3.10) together with (3.14) imply that the covariance matrix for $\left|\Psi_{G}(s)\right\rangle$ becomes

$$
G_{s}^{(a, b)}=U(s)_{c}^{a} G_{0}^{(c, d)} U(s)_{d}^{b}
$$

Eventually, the complexity of a target state in a basis independent way can be achieved by defining the relative covariance matrix

$$
\Delta_{b}^{a}=G_{T}^{(a, c)} G_{R(c, b)}^{-1}
$$

and make a proper choice for the cost function which by that, the complexity only depends on the eigenvalues of this matrix. One of these choices is the $\kappa=2$ in (3.8). Indeed one 
can choose a basis such that the $G_{R}$ becomes identity and then diagonalize $G_{T}$ using only transformations within the stabilizer subgroup $S t a_{G_{R}}$. Then $\kappa=2$ complexity is given by

$$
\mathcal{C}_{\kappa=2}\left(G_{T}, G_{R}\right)=\frac{1}{4} \operatorname{Tr}\left[|\log \Delta|^{2}\right] .
$$

It is worth to mention that the $\mathcal{C}_{\kappa=1}=\frac{1}{2} \operatorname{Tr}[|\log \Delta|]$ in general is different from the complexity obtained from $F_{1}$ in (3.8). The reason for that is $F_{1}$ can not be found from any well-defined metric. Despite $C_{\kappa=2}$, choosing the gates matters for $F_{1}$. One way to estimate the length of shortest path between the two states is to consider orthonormal symplectic group generators (with respect to Frobenius inner product) as our gates and calculate the length of the path that minimizes $F_{2}$ cost function, i.e. the linear path. Since this path is not necessary the minimal geodesic of $F_{1}$, then

$$
\mathcal{C}_{1} \leq \sum_{I}\left|Y^{I}\right|
$$

From now on, we take this upper bound as $\mathcal{C}_{1}$. Intriguingly, in the case of ground state of free bosonic quantum field theory, this approach gives the same answer as covariance matrix approach [15]. Last but not least, we need the dimensionless control functions to add them together. This can be achieved by introducing the new dimensionless position and momentum coordinates according to

$$
x_{\text {new }, \pm}=g_{s} x_{ \pm}, \quad p_{\text {new }, \pm}=\frac{p_{ \pm}}{g_{s}},
$$

where $g_{s}$ is a new gate scale. Accordingly, for each mode we have

$$
\lambda_{R, k} \equiv \lambda_{R}=\frac{\omega_{R}}{\delta g_{s}^{2}}, \quad \lambda_{k}=\frac{\omega_{k}}{\delta g_{s}^{2}} .
$$

Let us write the state (2.53) as following

$$
|\operatorname{cTFD}(t)\rangle=\hat{U}_{+}(1)\left|G_{0}\right\rangle_{+} \otimes \hat{U}_{-}(1)\left|G_{0}\right\rangle_{-}=e^{-i \hat{K}_{+}}\left|G_{0}\right\rangle_{+} \otimes e^{-i \hat{K}_{-}}\left|G_{0}\right\rangle_{-} .
$$

By choosing $\hat{\xi}_{ \pm}=\left(\hat{x}_{\text {new }, \pm}, \hat{p}_{\text {new }, \pm}\right)$ and noting to $(2.54)$, the unitary matrices $k_{(a, b),+}$ and $k_{(a, b),-}$ are given by

$$
k_{(a, b),+}=\alpha\left(\begin{array}{cc}
\lambda \sin ((\omega+\mu q) t) & \cos ((\omega+\mu q) t) \\
\cos ((\omega+\mu q) t) & -\frac{1}{\lambda} \sin ((\omega+\mu q) t) .
\end{array}\right), \quad k_{(a, b),-}=k_{(a, b),+}(\alpha \rightarrow-\alpha),
$$

with

$$
\lambda=m \omega / g_{s}^{2} .
$$

The commutator $\left[\hat{\xi}_{ \pm}^{a}, \hat{\xi}_{ \pm}^{b}\right]=i \Omega_{ \pm}^{a b}$ implies that

$$
\Omega_{+}^{a b}=\Omega_{-}^{a b}=\left(\begin{array}{cc}
0 & 1 \\
-1 & 0
\end{array}\right)
$$


and by that matrices $K_{ \pm}$become respectively,

$$
K_{+}=\alpha\left(\begin{array}{cc}
\cos ((\omega+\mu q) t) & -\frac{1}{\lambda} \sin ((\omega+\mu q) t) \\
-\lambda \sin ((\omega+\mu q) t) & -\cos ((\omega+\mu q) t)
\end{array}\right), \quad K_{-}=K_{+}(\alpha \rightarrow-\alpha) .
$$

Exponentiation of matrices $K_{ \pm}$gives the unitary matrices $U_{ \pm}(1)$,

$$
U_{+}(1)=\left(\begin{array}{ll}
u_{11} & u_{12} \\
u_{21} & u_{22}
\end{array}\right), \quad U_{-}(1)=U_{+}(1)(\alpha \rightarrow-\alpha),
$$

with

$$
\begin{aligned}
& u_{11}=\cosh (\alpha)+\cos ((\omega+\mu q) t) \sinh (\alpha), \quad u_{22}=u_{11}(\alpha \rightarrow-\alpha), \\
& u_{21}=\lambda^{2} u_{12}=-\lambda \sin ((\omega+\mu q) t) \sinh (\alpha) .
\end{aligned}
$$

Now, according to (3.26) and noting that

$$
G_{0,+}=G_{0,-}=\left(\begin{array}{cc}
\frac{1}{\lambda} & 0 \\
0 & \lambda
\end{array}\right)
$$

the eq. (3.15) implies that

$$
G_{\mathrm{TFD},+}(t)=\left(\begin{array}{c}
g_{11,+} g_{12,+} \\
g_{21,+} g_{22,+}
\end{array}\right), \quad G_{\mathrm{TFD},-}(t)=G_{\mathrm{TFD},+}(t)(\alpha \rightarrow-\alpha),
$$

with

$$
\begin{aligned}
& g_{11,+}=\frac{1}{\lambda}(\cosh (2 \alpha)+\cos ((\omega+\mu q) t) \sinh (2 \alpha)), \quad g_{22,+}=\lambda^{2} g_{11,+}(\alpha \rightarrow-\alpha), \\
& g_{12,+}=g_{21,+}=-\sin ((\omega+\mu q) t) \sinh (2 \alpha) .
\end{aligned}
$$

The last ingredient to construct the relative covariance matrix (3.16) is the covariance matrix for the unentangled states $\left|\Psi_{R, \pm}\right\rangle$. These states are the ground states of Hamiltonian (3.1) and their covariance matrices are given by

$$
G_{R,+}=G_{R,-}=\left(\begin{array}{cc}
\frac{1}{\lambda_{R}} & 0 \\
0 & \lambda_{R}
\end{array}\right)
$$

with

$$
\lambda_{R}=m \omega_{R} / g_{s}^{2} .
$$

Having all ingredients, (3.29) and (3.31), the relative covariance matrix (3.16) for right and left moving modes is given by

$$
\Delta_{+}(t)=\left(\begin{array}{c}
\Delta_{11,+} \Delta_{12,+} \\
\Delta_{21,+} \Delta_{22,+}
\end{array}\right), \quad \Delta_{-}(t)=\Delta_{+}(t)(\alpha \rightarrow-\alpha),
$$


with

$$
\begin{aligned}
& \Delta_{11_{+}}=\frac{\lambda_{R}}{\lambda}(\cosh (2 \alpha)+\cos ((\omega+\mu q) t) \sinh (2 \alpha)), \quad \Delta_{22_{+}}=\frac{\lambda^{2}}{\lambda_{R}^{2}} \Delta_{11_{+}}(\alpha \rightarrow-\alpha), \\
& \Delta_{21_{+}}=\lambda_{R}^{2} \Delta_{12_{+}}=-\lambda_{R} \sin ((\omega+\mu q) t) \sinh (2 \alpha) .
\end{aligned}
$$

Finally, according to (3.17) the $\kappa=2$ complexity for the single mode is given by

$$
\mathcal{C}_{\kappa=2}(t, q)=\frac{1}{4} \sum_{i=1}^{2}\left[\left(\log \Delta_{+}^{(i)}(t)\right)^{2}+\left(\log \Delta_{-}^{(i)}(t)\right)^{2}\right],
$$

where $\Delta_{ \pm}^{(i)}(t)$ are eigenvalues of $\Delta_{ \pm}(t)$ matrices (3.33),

$$
\begin{aligned}
& \Delta_{+}^{(1)}(t)=\frac{1}{A_{1}}\left(\lambda^{2} A_{2}+\lambda_{R}^{2} A_{3}-\left(\left(\lambda^{2} A_{2}+\lambda_{R}^{2} A_{3}\right)^{2}-A_{1}^{2}\right)^{\frac{1}{2}}\right), \\
& \Delta_{+}^{(2)}(t)=\frac{1}{A_{1}}\left(\lambda^{2} A_{2}+\lambda_{R}^{2} A_{3}+\left(\left(\lambda^{2} A_{2}+\lambda_{R}^{2} A_{3}\right)^{2}-A_{1}^{2}\right)^{\frac{1}{2}}\right), \\
& \Delta_{-}^{(i)}(t)=\Delta_{+}^{(i)}(t)(\alpha \rightarrow-\alpha),
\end{aligned}
$$

with

$$
A_{1}=2 \lambda \lambda_{R}, \quad A_{2}=\cosh (2 \alpha)-\cos ((\omega+\mu q) t) \sinh (2 \alpha), \quad A_{3}=A_{2}(\alpha \rightarrow-\alpha) .
$$

Having a complexity for single mode, (3.35), and noting that each mode $k$ is decoupled from the other modes, the complexity of the ground state of Hamiltonian (2.11) on a lattice with $N$ site is easily a sum over the complexity for each mode $k$,

$$
\mathcal{C}_{\kappa=2}(t, q)=\frac{1}{4} \sum_{k=0}^{N-1} \sum_{i=1}^{2}\left[\left(\log \Delta_{+}^{(i, k)}(t)\right)^{2}+\left(\log \Delta_{-}^{(i, k)}(t)\right)^{2}\right],
$$

where $\Delta_{ \pm}^{(i, k)}(t)$ are given by (3.36) upon substituting $\omega$ with $\omega_{k}$. In the subsequent subsections 3.1 and 3.2 we study the complexity (3.38) for the system with fixed size $L$. The same analysis for the continuum system will be presented in section 4 .

\subsection{Keeping the total size $L$ of the system fixed}

To explore the consequences of (3.38) for a system with fixed size $L$, we note that the only dimensionless parameters are $L / \beta$ and $\mu q L$. In figures 1 and 2 , we set $\lambda_{R}=1$ and the parameter $\mu q L$ is fixed but the temperature is changed. It is explained above that we consider the mass parameter $m L$ very small. One may expect that for this case the main contribution to the complexity comes from the zero mode. In the following, we will show that, independent from the value of $\mu q L$, this expectation is correct just for low temperatures. Indeed the contribution of the zero mode, in comparison with other modes, can be seen effectively in the limit $m \ll \omega_{R}$ where the eigenvalues (3.36) for this mode can be simplified to

$$
\Delta_{ \pm}^{(1)}(t) \approx 0, \quad \Delta_{ \pm}^{(2)}(t) \approx \frac{\omega_{R}}{m}(\cosh 2 \alpha \pm \cos [(m+\mu q) t] \sinh 2 \alpha) .
$$


In the limit $\beta(m+\mu q) \ll 1$, the above eigenvalues can be simplified more to

$$
\Delta_{+}^{(2)}(t) \approx \frac{4 \omega_{R}}{\beta m(m+\mu q)}-\frac{\omega_{R} t^{2}}{\beta m}(m+\mu q), \quad \Delta_{-}^{(2)}(t) \approx \frac{\omega_{R} t^{2}}{\beta m}(m+\mu q),
$$

which by using them and for the times $(m+\mu q) t \ll 1$, the contribution of zero mode to $\kappa=2$ complexity (3.35) becomes

$$
\mathcal{C}_{\kappa=2} \approx \frac{1}{4} \log ^{2}\left[\frac{\omega_{R}(m+\mu q)}{\beta m} t^{2}\right]+\frac{1}{4} \log ^{2}\left[\frac{4 \omega_{R}}{\beta m(m+\mu q)}\right] .
$$

This result is presented with the green curve in figures 1 and 2 and it exhibits that the contribution of zero mode for small times, $(m+\mu q) t \ll 1$ is proportional to $a_{1} \log ^{2}\left(a_{2} t^{2}\right)$. Besides that, it is clear from (3.36) that the biggest contribution of the zero mode happens at the time $t=\pi / 2(m+\mu q)$. This means that the maximum contribution of zero mode happens between times 0 and $\pi / 2(m+\mu q)$ where in the limits $(m+\mu q) \ll 1$ and $\beta(m+\mu q) \ll$ 1 it becomes

$$
\mathcal{C}_{\kappa=2}^{k=0}\left(\frac{\pi}{2(m+\mu q)}\right)-\mathcal{C}_{\kappa=2}^{k=0}(0) \approx \frac{1}{2} \log ^{2}\left[\frac{2 \omega_{R}}{\beta m(m+\mu q)}\right]-\frac{1}{4} \log ^{2}\left[\frac{4 \omega_{R}}{\beta m(m+\mu q)}\right] .
$$

Even though the zero mode contribution diverges at those limits but by paying attention to the figures 1 and 2, it is clear that at the decompactification limit i.e. high temperatures and independently from the charge, the contribution of the other modes dominate. More precisely, for higher temperatures, we observe saturation which happens through the presence of many modes which they contribute non-trivially to the sum (3.38). The transition between logarithmic growth regime and saturation regime is oscillatory, which occurs with a period of half of the circle's circumference, as if two wave packets were propagating on a circle in opposite directions, see figures 3 and 4 . For example when $m \ll \omega_{R}$ and $m \ll \mu q$, for the zero mode we have

$$
\Delta_{ \pm}^{(2)}(t) \approx \frac{\omega_{R}}{m} \operatorname{csch}\left(\frac{\beta \mu q}{2}\right)\left(\cosh \left(\frac{\beta \mu q}{2}\right) \pm \cos [\mu q t]\right) .
$$

We would like to emphasize again that above, $\lambda_{R}=1$ is considered since for this special value, it is proven that the optimal circuit does not mix the normal modes. Even though this simplicity of the optimal circuits might be lost when $\lambda_{R} \neq 1$, just to make the numerical problem tractable, we still assume that there is no mixing between the normal modes along the circuit. The same analysis as before for $\lambda_{R} \neq 1$ is presented in figures 5 and 6 which they show the validity of the results for $\lambda_{R}=1$ also in this case.

To close this section, we show the asymmetry between the effect of mass and charge times chemical potential in figure 7 .

\subsection{Keeping the lattice spacing fixed with increasing $N$}

What we have done in previous subsection was introducing a UV-regularization and mode decomposition in which the Hamiltonian of a continuous quantum many-body system became a sum over independent harmonic oscillators (bosonic modes). The latter one implies 

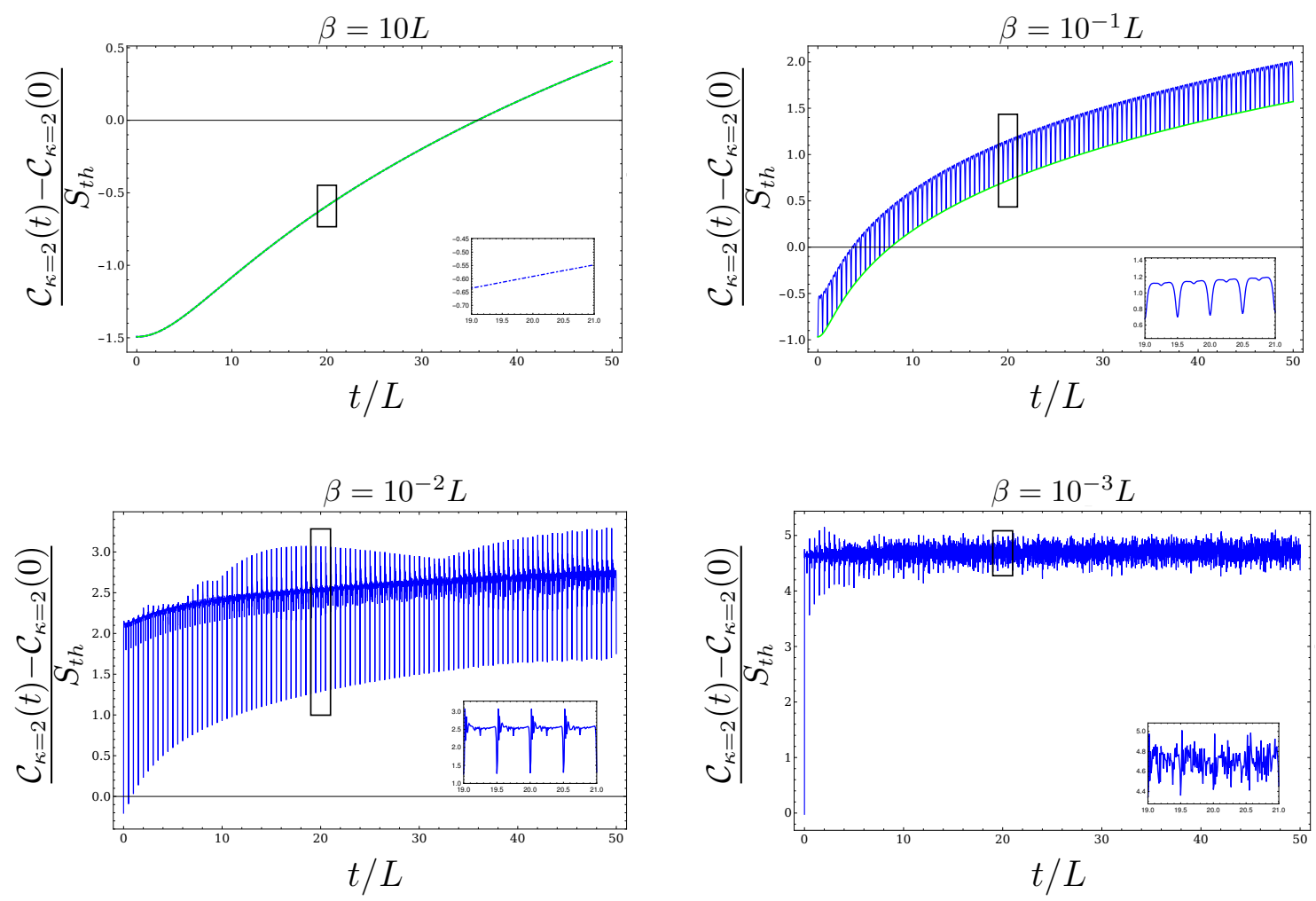

Figure 1. All mode contribution to time dependence of $C_{\kappa=2}$ with the initial value subtracted for the neutral TFD at zero time on a circle with circumference $L$ with $\omega_{R}=1 / L, m=10^{-5} / L$, $\mu q=10^{-5} / L$ and increasing temperatures $T=1 / \beta$. We use 1501 lattice sites on each side.

the complexity for QFT is found by simply adding up contributions for each bosonic mode. One can use an interesting different regularization which is based on the continuous multiscale entanglement renormalization ansatz (cMERA). In cMERA approach, a UV regulator is introduced such that the ground state for large momenta $|p|>\Lambda$ behaves as a product state i.e. the ground state of ultralocal Hamiltonian (3.1). We extend this regularization also for the cTFD state. In next section, we will study the continuous system on the line with details. For the moment, the figure 8 demonstrates that the decompactification limit of the circle ( $\delta$ is fixed and $N \rightarrow \infty$ ) quantitatively reproduces the results on an infinite line, and provides an example of the use of cMERA-inspired techniques in the context of complexity.

\section{Working with the infinite system}

To find the complexity in the continuum limit, we use the cMERA inspired regularization. Let us introduce a continuous label $p \in\left[-\frac{\pi}{\delta}, \frac{\pi}{\delta}\right]$ defined as

$$
p=\frac{2 \pi}{N} \frac{k}{\delta}
$$



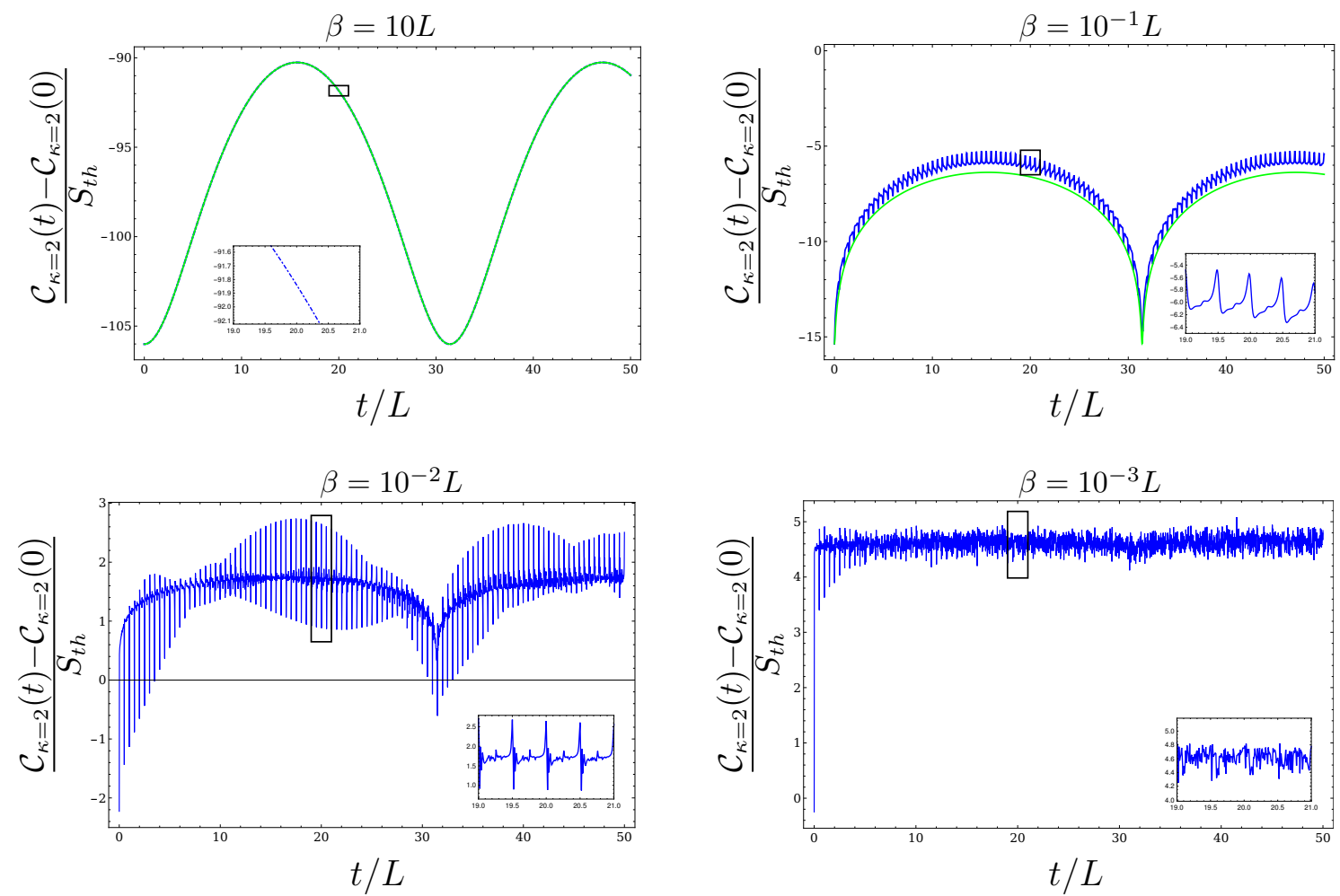

Figure 2. All mode contribution to time dependence of $C_{\kappa=2}$ with the initial value subtracted for the neutral TFD at zero time on a circle with circumference $L$ with $\omega_{R}=1 / L, m=10^{-5} / L$, $\mu q=10^{-1} / L$ and increasing temperatures $T=1 / \beta$. We use 1501 lattice sites on each side.
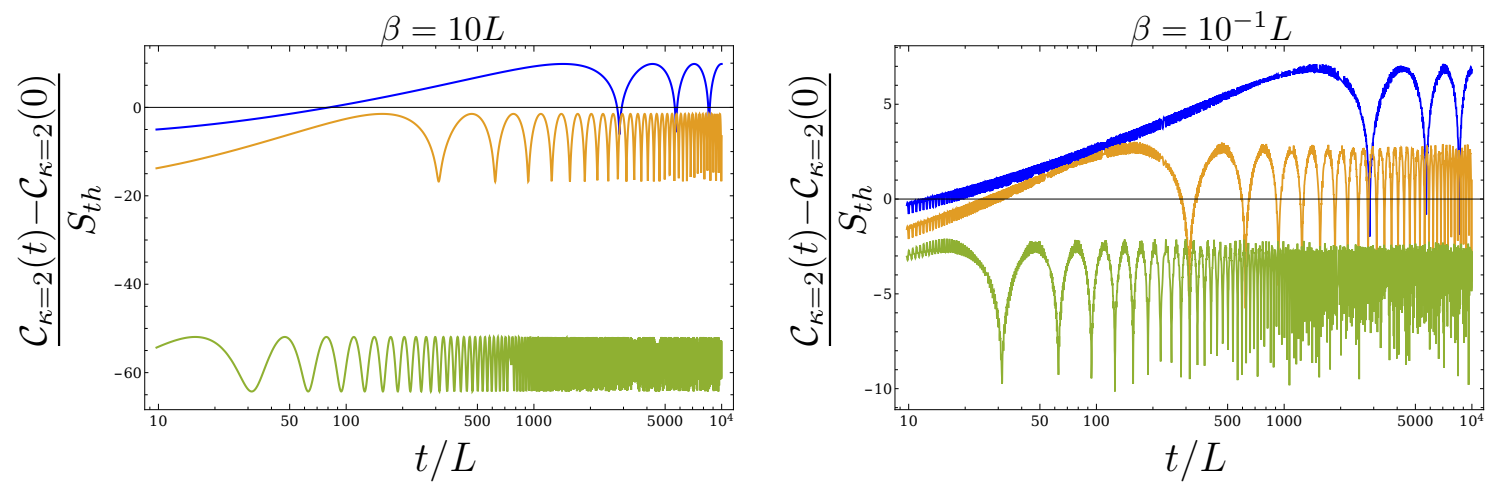

Figure 3. Time dependence of $\kappa=2$ complexity with the initial value subtracted for TFD state on a circle with circumference $L$ with $\omega_{R}=1 / L, m=10^{-4} / L, \lambda_{R}=1, \beta=10 L$ (left), $\beta=10^{-1} L$ (right) and different increasing the chemical potential from $10^{-3} / L$ (blue) to $10^{-1} / L$ (green). We use 1501 lattice sites on each side. We see that complexity grows as $\log ^{2}(t / L)$ up to times of the order of $1 /(m+\mu q)$ when it starts oscillating around the saturated value. The zero mode is the source for this logarithmic growth but by increasing the temperature this behavior is lost. 

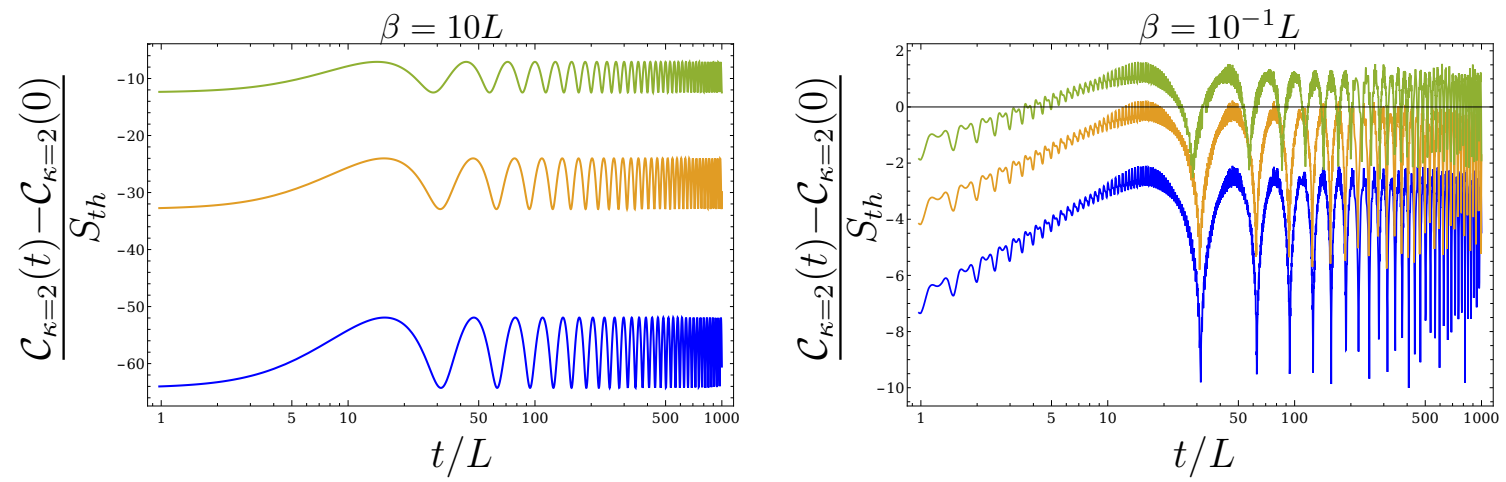

Figure 4. Time dependence of $\kappa=2$ complexity with the initial value subtracted for TFD state on a circle with circumference $L$ with $\omega_{R}=1 / L, \mu q=10^{-1} / L, \beta=10 L$ (left), $\beta=10^{-1} / L$ (right) and different increasing the masses, from $10^{-4} / L$ (blue) to $10^{-2} / L$ (green). We use 1501 lattice sites on each side. We see that complexity grows as $\log ^{2}(t / L)$ up to times of the order of $1 /(m+\mu q)$ when it starts oscillating around the saturated value. The zero mode is the source for this logarithmic growth but by increasing the temperature this behavior is lost.

This definition for continuous momentum $p$ implies that $\omega_{p},(2.12)$, becomes

$$
\omega_{p}^{2}=m^{2}+\frac{4}{\delta^{2}} \sin ^{2}\left(\frac{p \delta}{2}\right) .
$$

The continuity of momentum $p$ implies that we have taken the limit of large chain, $L \gg \delta$ (i.e. $N \gg 1$ while keeping $\delta$ fixed). Since energy of all modes is less than the UV cut off $\Lambda$ and $\Lambda$ itself is much less than $\pi / \delta$, therefore $\omega_{p}(4.2)$ is simplified to ${ }^{3}$

$$
\omega_{p}=\sqrt{m^{2}+p^{2}} \text {. }
$$

In the following, we consider the complex scalar theory (2.1) in d-dimensional flat spacetime and with mass $\mathrm{m}$. In this case, one may also modify the gate sale. For d-dimensional spacetime, the relations in (2.5) become

$$
\mathbf{x}_{a}=\Phi\left(x_{a}\right) \delta^{d / 2}, \quad \mathbf{p}_{a}=\Pi\left(x_{a}\right) \delta^{d / 2-1},
$$

which they imply that in going from the lattice to the continuum expressions, we should absorb a factor of $1 / \delta^{d-1}$ into control functions and also define the gate scale as follows

$$
\omega_{R, g} \equiv \delta g_{s}^{2}
$$

Accordingly, the dimensionless ratios in (3.20) change to

$$
\lambda_{R}=\frac{\omega_{R}}{\omega_{R, g}}, \quad \lambda=\frac{\omega_{k}}{\omega_{R, g}} .
$$

Furthermore, in order to make contact with holography, we are primarily interested in CFTs (i.e. massless models). However as we have pointed above, the massless limit of (3.36) is

\footnotetext{
${ }^{3}$ One may also demand that the frequency of the oscillator is continuous at the transition point, i.e. $\omega_{p=\Lambda}=\omega_{R}$ where $\omega_{p}$ was defined in (4.2).
} 

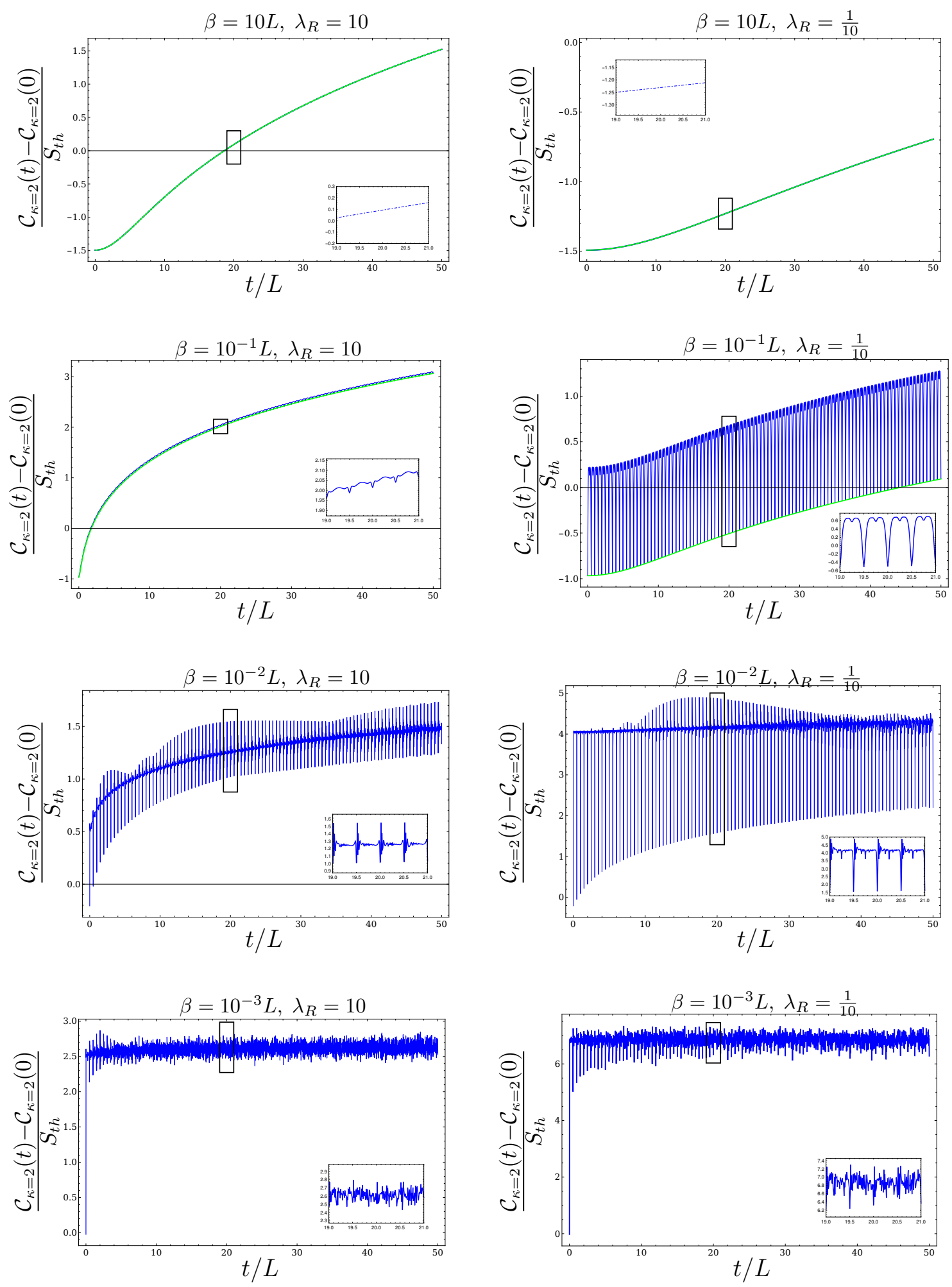

Figure 5. All mode contribution to time dependence of $C_{\kappa=2}$ with the initial value subtracted for the neutral TFD at zero time on a circle with circumference $L$ with $\omega_{R}=1 / L, m=10^{-5} / L$, $\mu q=10^{-5} / L$. We use 1501 lattice sites on each side. 

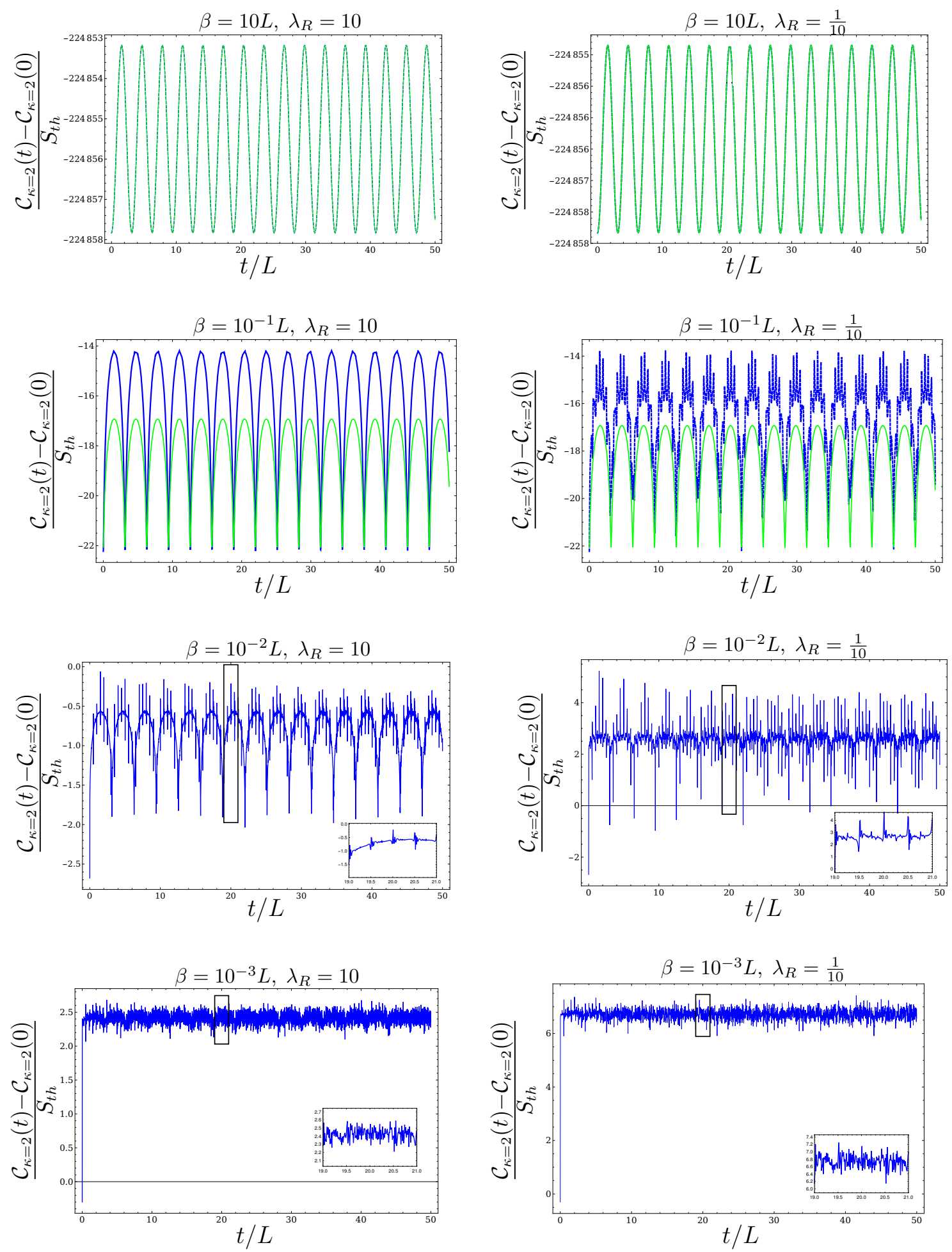

Figure 6. All mode contribution to time dependence of $C_{\kappa=2}$ with the initial value subtracted for the neutral TFD at zero time on a circle with circumference $L$ with $\omega_{R}=1 / L, m=10^{-5} / L$, $\mu q=1 / L$. We use 1501 lattice sites on each side. 

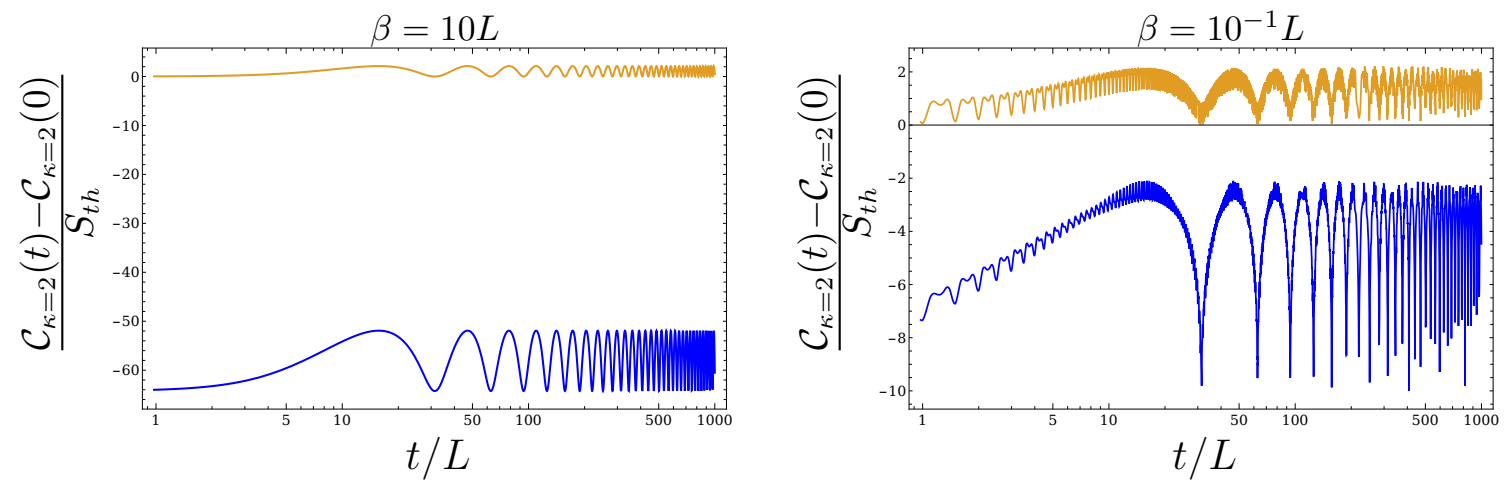

Figure 7. Time dependence of $\kappa=2$ complexity with the initial value subtracted for TFD state on a circle with circumference $L$ with $\omega_{R}=1 / L,\left(m=10^{-4} / L, \mu q=10^{-1} / L\right)$ (blue), ( $\left.m=10^{-1} / L, \mu q=10^{-4} / L\right)$ (brown). Left: $\beta=10 L$ and Right: $\beta=10^{-1} L$. We use 1501 lattice sites on each side.
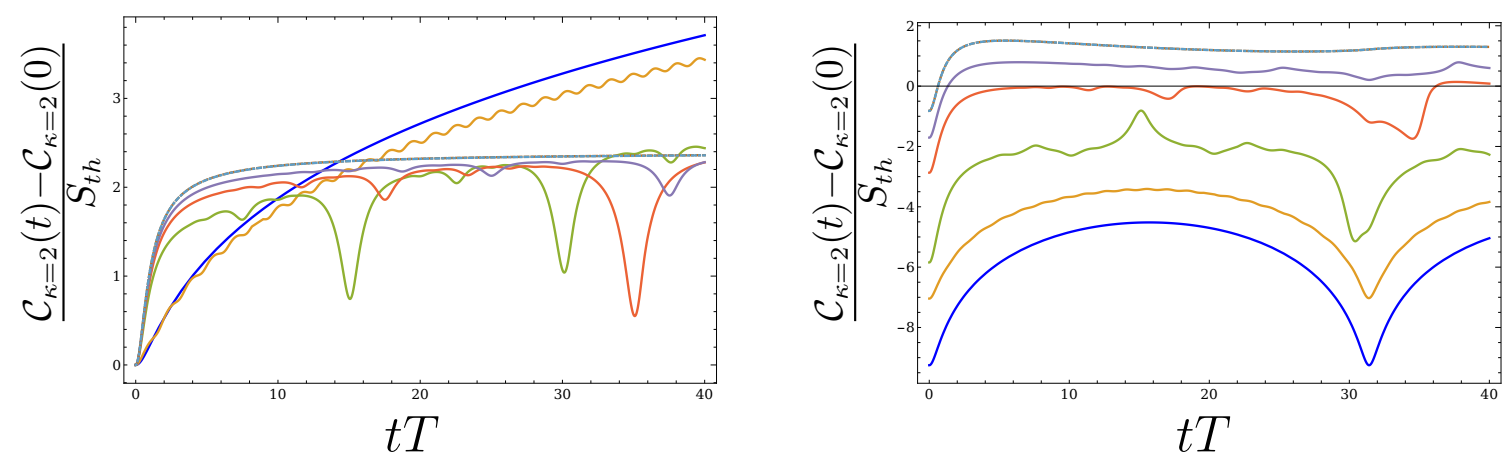

Figure 8. Time dependence of $\kappa=2$ complexity with the initial value subtracted for TFD state with fixed lattice spacing $\delta, m=10^{-6} / \delta, \beta=10 \delta$ and $\omega_{R}=1 / \delta, \mu q=0$ (left) and $\mu q=10^{-2} / \delta$ (right). The solid curves represents the theory on a circle with the total number of cites $N=1$ (blue), $N=31$ (brown), $N=301$ (green), $N=701$ (red), $N=1501$ (purple). The solid blue and brown curves are scaled with $1 / 5$ for the cTFD state. The dashed brown and dotted dashed blue curve represent the result respectively for the cMERA inspired technique with UV cutoff $\Lambda=1 / \delta$ and the one obtained directly for a theory on an infinite line.

ill-defined since the zero mode gives a divergent contribution. We regulate this divergence by instead working with a small but non-zero mass. Moreover, in order to UV regulate our results for the complexity, one can subtract its value at the initial time $t=0$. Accordingly, based on covariance matrix approach (3.17), the desired complexities in the continuum limit reads

$$
\begin{aligned}
\mathcal{C}_{\kappa=2}(t) & =\operatorname{vol} \int_{p \leq \Lambda} \frac{d^{d-1} p}{(2 \pi)^{d-1}} \frac{1}{4} \sum_{i=1}^{2}\left[\left(\log \Delta_{+}^{(i)}(t, p)\right)^{2}+\left(\log \Delta_{-}^{(i)}(t, p)\right)^{2}\right] \\
\mathcal{C}_{2}(t) & =\operatorname{vol} \int_{p \leq \Lambda} \frac{d^{d-1} p}{(2 \pi)^{d-1}}\left(\frac{1}{4} \sum_{i=1}^{2}\left[\left(\log \Delta_{+}^{(i)}(t, p)\right)^{2}+\left(\log \Delta_{-}^{(i)}(t, p)\right)^{2}\right]\right)^{\frac{1}{2}}, \\
\mathcal{C}_{1}(t) & =\operatorname{vol} \int_{p \leq \Lambda} \frac{d^{d-1} p}{(2 \pi)^{d-1}} \frac{1}{2} \sum_{i=1}^{2}\left[\left|\log \Delta_{+}^{(i)}(t, p)\right|+\left|\log \Delta_{-}^{(i)}(t, p)\right|\right]
\end{aligned}
$$


where $\Delta_{ \pm}^{(i)}(t, p)$ are obtained by substituting

$$
\lambda \rightarrow \lambda_{p}, \quad \omega \rightarrow \omega_{p}
$$

in eigenvalues (3.36). In the first view, one may suspect that these integrals will produce UV divergences. But these potential divergences are eliminated, because we have powers of $e^{-\beta \Lambda}$ competing against (positive) powers of $\Lambda$ in these contributions, and so they actually vanish in the limit of $\Lambda \rightarrow \infty$. According to this fact, we remove the UV regulator and integrating all the way to infinite momenta. But the above integrals have IR divergences which they are encoded in the "vol" coefficients. These IR divergences can be remove by dividing the change of complexity with thermal entropy

$$
\left.S_{\mathrm{th}}=\frac{\operatorname{vol}}{\beta^{d-1}} \frac{\Omega_{d-2}}{(2 \pi)^{d-1}} \int_{0}^{\infty} d u u^{d-2}\left[\frac{\sqrt{u^{2}+\beta^{2} m^{2}}+\beta \mu q}{e^{\sqrt{u^{2}+\beta^{2} m^{2}}+\beta \mu q}-1}-\log \left(1-e^{-\sqrt{u^{2}+\beta^{2} m^{2}}-\beta \mu q}\right)\right)\right],
$$

where $\Omega_{d-2}$ is the volume of a $(d-2)$-sphere, i.e. $\Omega_{d-2}=2 \pi^{\frac{d-1}{2}} / \Gamma\left(\frac{d-1}{2}\right)$. Last but not least, to take into account the reference scale together with massless limit, it will be useful to define dimensionless variables

$$
\tilde{t}=\frac{t}{\beta}, \quad \tilde{k}=\beta k, \quad \tilde{\gamma}=\frac{1}{\beta \omega_{R}}, \quad \tilde{Q}=\mu q \beta .
$$

\subsection{Complexity of formation: A prob for prefer cost function}

In this subsection we study the complexity of formation for cTFD state (2.40) carefully to see which cost function is consistent with the UV structure and third law of holographic complexity [29]. The complexity of formation is indeed the extra complexity required to prepare the two copies of a complex scalar field theory in the cTFD state compared to simply preparing each of the copies in the vacuum state, i.e. $\Delta \mathcal{C}=\mathcal{C}(0)-\left.\mathcal{C}(0)\right|_{\beta \rightarrow \infty}$. For the case where the frequency of all modes $\omega_{p}$ is much less than the reference scale $\omega_{R}$, the eigenvalues (3.36) can be simplified to

$$
\Delta_{ \pm}^{(1)}(t) \approx 0, \quad \Delta_{ \pm}^{(2)}(t) \approx \frac{\omega_{R}}{\omega_{p}}\left(\cosh 2 \alpha_{p} \pm \cos \left[\left(\omega_{p}+\mu q\right) t\right] \sinh 2 \alpha_{p}\right) .
$$

It is worth noting that very high frequency contributions are exponentially suppressed and so in fact, we only need to consider $\omega_{R} \gg T$. In this regime, the complexity of formation for the ones in (4.7) becomes

$$
\begin{aligned}
\Delta \mathcal{C}_{\kappa=2} & =\operatorname{vol} \int_{p \leq \Lambda} \frac{d^{d-1} p}{(2 \pi)^{d-1}} 2 \alpha_{p}^{2} \\
\Delta \mathcal{C}_{2} & =\operatorname{vol} \int_{p \leq \Lambda} \frac{d^{d-1} p}{(2 \pi)^{d-1}} \sqrt{\frac{1}{2} \log ^{2}\left(\frac{\omega_{P}}{\omega_{R}}\right)+2 \alpha_{p}^{2}}-\frac{1}{\sqrt{2}}\left|\log \frac{\omega_{p}}{\omega_{R}}\right| \\
\Delta \mathcal{C}_{1} & =\operatorname{vol} \int_{p \leq \Lambda} \frac{d^{d-1} p}{(2 \pi)^{d-1}}\left(\left|\frac{1}{2} \log \frac{\omega_{p}}{\omega_{R}}-\alpha_{p}\right|+\left|\frac{1}{2} \log \frac{\omega_{p}}{\omega_{R}}+\alpha_{p}\right|-\left|\log \frac{\omega_{p}}{\omega_{R}}\right|\right) .
\end{aligned}
$$


These complexities are presented in figure 9. To probe which of them is consistent with UV divergences in holographic results, let us focus for the moment on the vacuum state $\left(\alpha_{p} \rightarrow 0\right)$. According to (4.11) for this state we have

$$
\mathcal{C}_{\kappa=2}=\frac{1}{4} \sum_{\left[p_{i}\right]=0}^{N-1}\left(\log \frac{\omega_{\vec{p}}}{\omega_{R}}\right)^{2}, \quad \mathcal{C}_{2}=\frac{1}{2} \sqrt{\sum_{\left[p_{i}\right]=0}^{N-1}\left(\log \frac{\omega_{\vec{p}}}{\omega_{R}}\right)^{2}}, \quad \mathcal{C}_{1}=\frac{1}{2} \sum_{\left[p_{i}\right]=0}^{N-1}\left|\log \frac{\omega_{\vec{p}}}{\omega_{R}}\right|
$$

with $p_{i}$ 's are the components of the momentum vector $\vec{p}=\left(p_{1}, p_{2}, \ldots, p_{d-1}\right)$ and the total number of oscillators is $N^{d-1}=V / \delta^{d-1}$. Therefore, contribution of UV modes i.e. $\omega_{\vec{p}} \sim 1 / \delta$ to (4.13) become

$$
\mathcal{C}_{\kappa=2} \sim \frac{V}{\delta^{d-1}}\left(\log \frac{1}{\delta \omega_{R}}\right)^{2}, \quad \mathcal{C}_{2} \sim\left(\frac{V}{\delta^{d-1}}\right)^{1 / 2} \log \left(\frac{1}{\delta \omega_{R}}\right), \quad \mathcal{C}_{1} \sim \frac{V}{\delta^{d-1}}\left|\log \frac{1}{\delta \omega_{R}}\right|
$$

The leading divergence appearing in CA proposal takes the

$$
\mathcal{C}_{\text {holography }} \sim \frac{V}{\delta^{d-1}} \log \left(\frac{l}{\tilde{\alpha} \delta}\right),
$$

where $\delta$ is the short-distance cut-off scale in the boundary CFT, $l$ is the AdS curvature scale of the bulk spacetime, and $\tilde{\alpha}$ is an arbitrary (dimensionless) coefficient which fixes the normalization of the null normals on the boundary of the WDW patch. Since $\mathcal{C}_{\text {holography }}$ is a quantity which is to be defined in the boundary CFT, it should not depend on the bulk AdS scale. One can eliminate this factor with the freedom in choosing $\tilde{\alpha}=\tilde{\omega}_{R} l$ where $\tilde{\omega}_{R}$ is some arbitrary frequency. In this case (4.15) is simplified to

$$
\mathcal{C}_{\text {holography }} \sim \frac{V}{\delta^{d-1}} \log \left(\frac{1}{\delta \tilde{\omega}_{R}}\right)
$$

which interestingly agrees with $\mathcal{C}_{1}$ in (4.14). It is discussed [32] that the CA proposal which leds to (4.15) does not have a reparameterization invariance of null boundary coordinates. To recover this symmetry one can consider a new boundary term which intriguingly adding it also removes that UV logarithmic divergence. The $\mathcal{C}_{1}$ complexity (4.14) agrees with holography also in the presence of this new boundary term if, for example $\omega_{R}$ is set by the cut-off scale i.e. $\omega_{R} \sim 1 / \delta$.

We would like to mention here that according to the above discussion, it seems $\mathcal{C}_{1}$ complexity (4.13) for ground state agrees with the holographic proposal. But by carefully noting to figure 9 , we see that $\mathcal{C}_{1}$ complexity is in contrast with the third law of holographic complexity [9]. The third law of holographic complexity expresses that the complexity of formation for a charged black hole diverges in extremal limit. The origin of this mismatch can be understood by evaluating carefully that the sign changes inside each argument of the logarithms in (4.13). Let us concentrate on the massless theory for the moment. In this case, $\frac{1}{2} \log \frac{p}{\omega_{R}}+\alpha_{p}$ changes sign at a value $p_{c}$ given by

$$
p_{c} \operatorname{coth}\left(\frac{p_{c}+\mu q}{4 T}\right)=\omega_{R}
$$



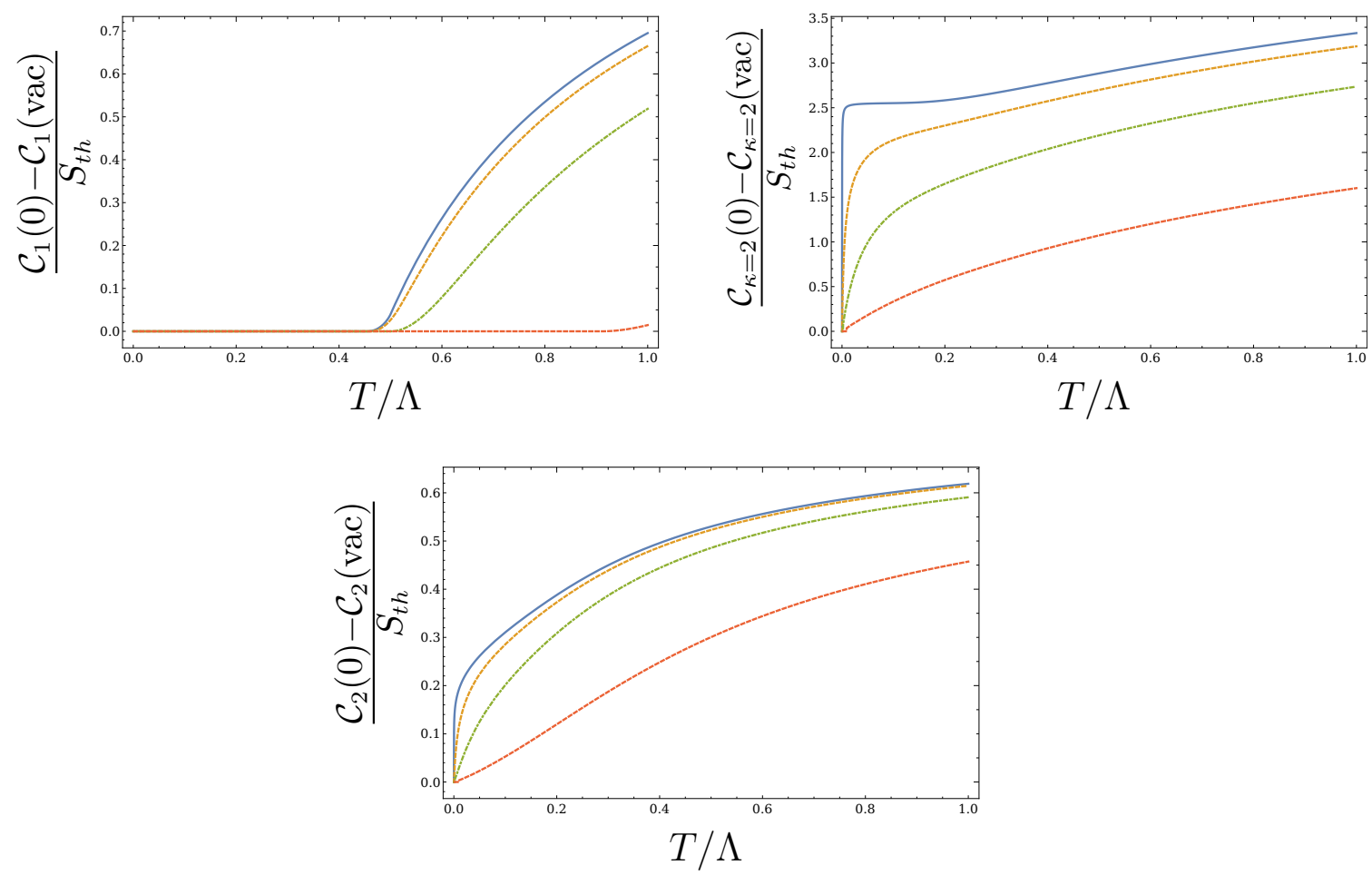

Figure 9. $\left(\mathcal{C}_{1}, \mathcal{C}_{\kappa=2}, \mathcal{C}_{2}\right)$ complexities of formation normalized by the entropy for $\mu q=10^{-5} \Lambda$ (blue), $10^{-2} \Lambda$ (dashed orange), $10^{-1} \Lambda$ (dotted dashed green), $\Lambda$ (dashed red) and $d=1+1, m=$ $10^{-5} \Lambda, \omega_{R}=2 \Lambda$. For small temperatures, the profile of the ratio of the complexity of formation over the entropy becomes small. For higher temperatures, it develops a dependence on the temperature and the cutoff scale $\Lambda$. The nontrivial profile of the ratio of the complexity of formation to the entropy contrasts with the holographic results of ref. [9].

There are two important limits to the above equation: when $p_{c}+\mu q$ is very small and when $p_{c}$ is close to the cutoff scale $\Lambda$. Solving for the temperature in these two regimes, we find

$$
T_{\mathrm{c}_{1}}=\frac{(\Lambda+\mu q)}{2} \frac{1}{\log \left(\frac{\omega_{R}+\Lambda}{\omega_{R}-\Lambda}\right)}, \quad T_{\mathrm{c}_{2}}=\frac{\omega_{R}}{4}\left(1+\frac{\mu q}{p_{c}}\right) .
$$

For $T<T_{\mathrm{c}_{1}}$, the arguments of all of the absolute values in (4.12) are negative which implies that the complexity of formation is identically zero, $\Delta \mathcal{C}_{1}\left(T<T_{\mathrm{cl}}\right)=0$. For temperature within the range $T_{\mathrm{c}_{1}}<T<T_{\mathrm{c}_{2}}$, there is a single solution $p_{c}$ to the (4.17) in the range $[0, \Lambda]$. We find that for $p<p_{c}$ the argument of the second absolute value is negative, and for $p>p_{c}$ the argument is positive. The complexity of formation in this situation is found by integrating only over modes larger than $p_{c}$,

$$
\Delta \mathcal{C}_{1}\left(T_{\mathrm{c}_{1}}<T<T_{\mathrm{c}_{2}}\right)=\operatorname{vol} \frac{\Omega_{d-2}}{(2 \pi)^{d-1}} \int_{p_{c}}^{\Lambda} d p p^{d-2}\left(2 \alpha_{p}-\log \frac{p}{\omega_{R}}\right)
$$

Finally, if the temperature is bigger than $T_{\mathrm{c}_{2}}$, we find that $\frac{1}{2} \log \frac{p}{\omega_{R}}+\alpha_{p}$ is always positive 

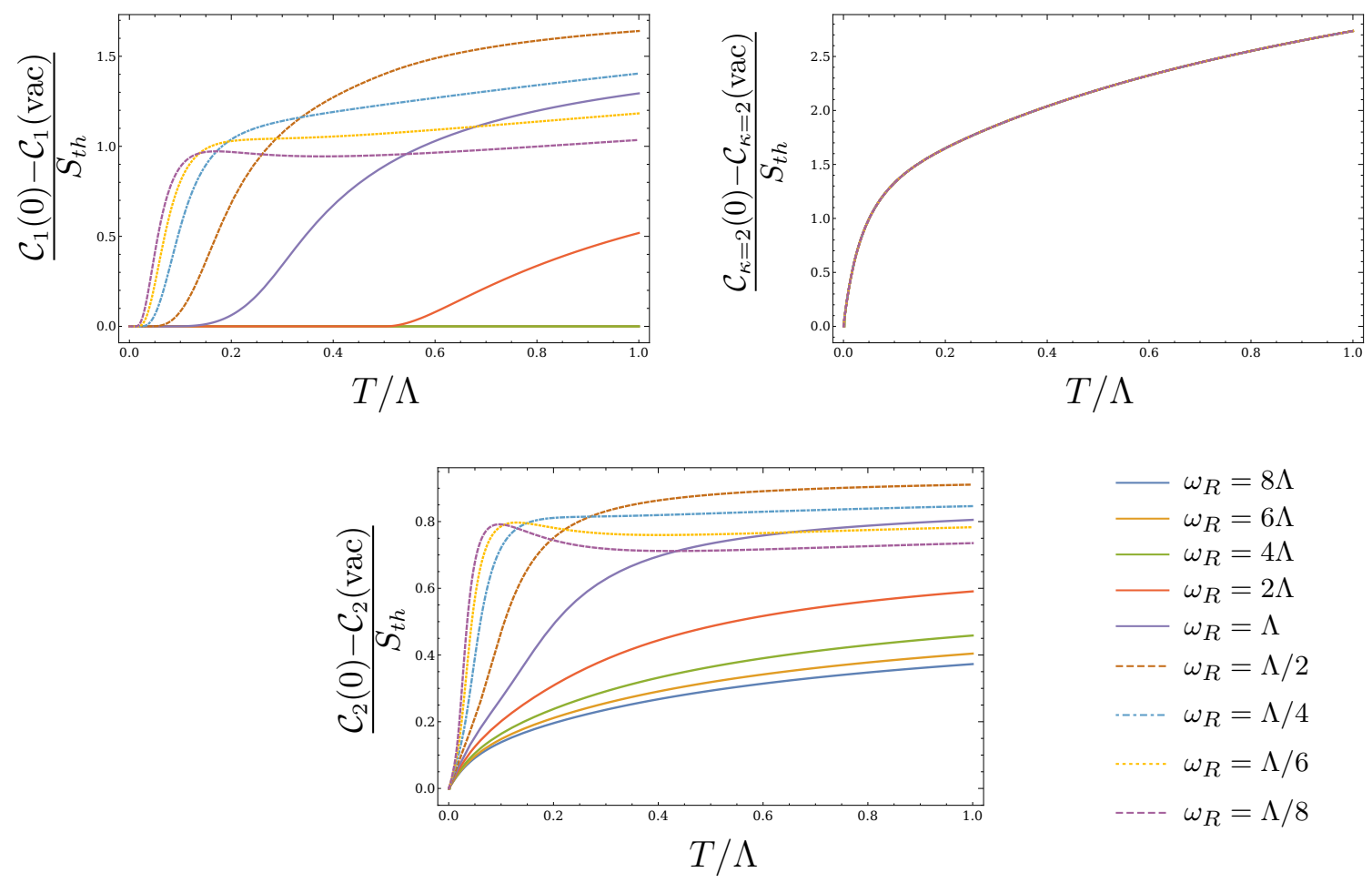

Figure 10. $\mathcal{C}_{1}, \mathcal{C}_{\kappa=2}, \mathcal{C}_{2}$ complexities of formation normalized by the entropy for different values of $\omega_{R}$ and $d=1+1, m=10^{-5} \Lambda, \mu q=10^{-1} \Lambda$. For small temperatures, the profile of the ratio of the complexity of formation over the entropy becomes small. For higher temperatures, it develops a dependence on the temperature and the cutoff scale $\Lambda$. The nontrivial profile of the ratio of the complexity of formation to the entropy contrasts with the holographic results of ref. [9].

in the range of momenta $[0, \Lambda]$. Therefore, we have

$$
\Delta \mathcal{C}_{1}\left(T>T_{\mathrm{C}_{2}}\right)=\operatorname{vol} \frac{\Omega_{d-2}}{(2 \pi)^{d-1}} \int_{0}^{\Lambda} d p p^{d-2}\left(2 \alpha_{p}-\log \frac{p}{\omega_{R}}\right) .
$$

Therefore for small temperatures with respect to the cutoff scale, we find that the complexity of formation is exactly zero. For higher temperatures, there are some nontrivial cancellations between the circuits that introduce some dependence on $\mathrm{T}$ and $\Lambda$ that contrasts with the holographic results of ref. [9]. To complete this discussion, we also present the same analysis for different $\omega_{R}$ in figure 10 and for $d=3+1$ in figure 11 .

Based on the above contradiction with holography, we should use another cost function which also gives the same UV divergence as $\mathcal{C}_{1}$. It is argued that [29] the basis-dependent $L^{1}$ norm in (3.8) is a preferred choice. According to (2.45) and (2.48), in the $L R$ basis, two copies of the physical degrees of freedom entangled in the cTFD state and in the diagonal or \pm basis the cTFD state factorizes. For a single mode, these basis are related by a simple rotation

$$
\left(\begin{array}{l}
\hat{x}_{+} \\
\hat{p}_{+} \\
\hat{x}_{-} \\
\hat{p}_{-}
\end{array}\right)=R_{4}\left(\begin{array}{l}
\hat{x}_{L} \\
\hat{x}_{R} \\
\hat{p}_{L} \\
\hat{p}_{R}
\end{array}\right), \quad \text { with } \quad R_{4}=\frac{1}{\sqrt{2}}\left(\begin{array}{cccc}
1 & 1 & 0 & 0 \\
0 & 0 & 1 & 1 \\
1 & -1 & 0 & 0 \\
0 & 0 & 1 & -1
\end{array}\right) .
$$



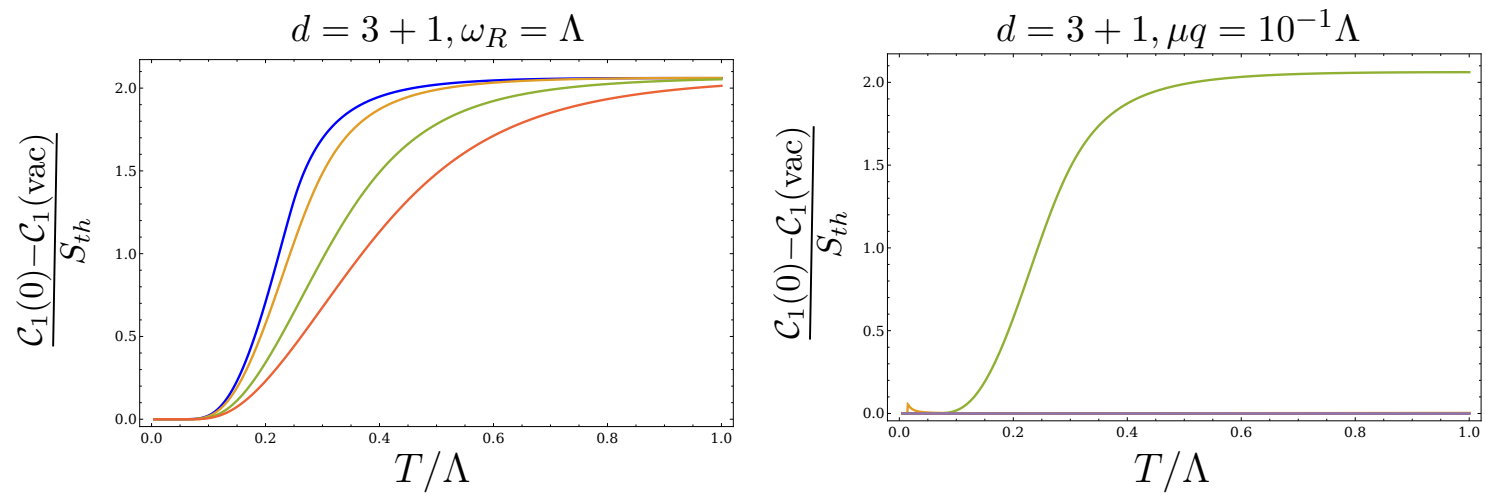

Figure 11. Left: $\mathcal{C}_{1}$ complexity of formation normalized by the entropy for $\mu q=0$ (blue), $10^{-1} \Lambda$ (brown), $\Lambda / 2$ (green), $\Lambda$ (red) and $d=3+1, m=10^{-5} \Lambda, \omega_{R}=\Lambda$. For small temperatures, the profile of the ratio of the complexity of formation over the entropy becomes small. For higher temperatures, it develops a dependence on the temperature and the cutoff scale $\Lambda$. The nontrivial profile of the ratio of the complexity of formation to the entropy contrasts with the holographic results of [9]. Right: $\mathcal{C}_{1}$ complexity of formation normalized by the entropy for $\omega_{R}=10^{-2} \Lambda$ (brown), $\Lambda$ (green), $10^{4} \Lambda$ (purple) and $d=3+1, m=10^{-5} \Lambda, \mu q=10^{-1} \Lambda$. For small and large values of reference scale, the profile of the ratio of the complexity of formation over the entropy is almost very small. For high temperatures, for reference scale in order of UV cutoff it develops a dependence on the temperature and the cutoff scale $\Lambda$.

Accordingly, the two-point function transforms as follows

$$
G^{( \pm)}=R_{4} G^{(L R)} R_{4}^{\mathrm{T}}
$$

which it implies that if we have a circuit acting in the diagonal basis as $G_{T}^{( \pm)}=$ $U^{( \pm)} G_{R}^{( \pm)} U^{( \pm), \mathrm{T}}$, then the same circuit in the $L R$ basis is

$$
G_{\mathrm{T}}^{(L R)}=U^{(L R)} G_{R}^{(L R)}\left(U^{(L R)}\right)^{\mathrm{T}} \quad \text { with } \quad U^{(L R)}=R_{4}^{\mathrm{T}} U^{( \pm)} R_{4} .
$$

For the $F_{1}$ cost function, we also have considerable freedom in choosing the basis of generators $K_{I}$ in (3.4). One can impose that the generators be orthonormal under the inner product inducing the $F_{2}$ cost function. For the single degree of freedom the generator group is $\operatorname{Sp}(2, R)=\mathrm{SL}(2, R)$, whose algebra is given by the traceless matrices $K_{I} \in T_{1}, T_{2}, T_{3}$,

$$
T_{1}=\left(\begin{array}{cc}
1 & 0 \\
0 & -1
\end{array}\right), \quad T_{2}=\left(\begin{array}{cc}
0 & 0 \\
-\sqrt{2} & 0
\end{array}\right), \quad T_{1}=\left(\begin{array}{cc}
0 & \sqrt{2} \\
0 & 0
\end{array}\right),
$$

with

$$
\left[T_{2}, T_{1}\right]=2 T_{2}, \quad\left[T_{1}, T_{3}\right]=2 T_{3}, \quad\left[T_{2}, T_{3}\right]=2 T_{1} .
$$

Exponentiating these generators yields the group elements that will serve as the elementary gates used in the construction of quantum circuits

$$
U_{T_{1}}=e^{\epsilon T_{1}}=\left(\begin{array}{cc}
e^{\epsilon} & 0 \\
0 & e^{-\epsilon}
\end{array}\right), \quad U_{T_{2}}=e^{\epsilon T_{2}}=\left(\begin{array}{cc}
1 & 0 \\
-\sqrt{2} \epsilon & 1
\end{array}\right), \quad U_{T_{3}}=e^{\epsilon T_{3}}=\left(\begin{array}{cc}
1 & \sqrt{2} \epsilon \\
0 & 1
\end{array}\right),
$$


with $\epsilon$ is a real parameter with $\epsilon \ll 1$. According to $(3.29)$ for $G_{T}^{( \pm)}=U^{( \pm)} G_{R}^{( \pm)}\left(U^{( \pm)}\right)^{T}$, it is easy to see

$$
U_{+}=\left(e^{T_{1,+}}\right)^{\left(\alpha-\frac{1}{2} \log \frac{\lambda}{\lambda_{R}}\right)}, \quad U_{-}=\left(e^{T_{1,-}}\right)^{\left(-\alpha-\frac{1}{2} \log \frac{\lambda}{\lambda_{R}}\right)} .
$$

The corresponding matrix $U$ in $L R$ bases is $U^{(L R)}=R_{4}^{T} U^{( \pm)} R_{4}$ where

$$
U^{( \pm)}=e^{K^{( \pm)}}, \quad K^{( \pm)}=\left(\begin{array}{cccc}
\alpha-\frac{1}{2} \log \frac{\lambda}{\lambda_{R}} & 0 & 0 & 0 \\
0 & -\alpha+\frac{1}{2} \log \frac{\lambda}{\lambda_{R}} & 0 & 0 \\
0 & 0 & -\alpha-\frac{1}{2} \log \frac{\lambda}{\lambda_{R}} & 0 \\
0 & 0 & 0 & \alpha+\frac{1}{2} \log \frac{\lambda}{\lambda_{R}}
\end{array}\right)
$$

After a short computation, one can find $U^{(L R)}$ which by knowing that, the generator $K^{(L R)}$ in LR basis become

$$
K^{(L R)}=\left(\begin{array}{cccc}
-\frac{1}{2} \log \frac{\lambda}{\lambda_{R}} & \alpha & 0 & 0 \\
\alpha & -\frac{1}{2} \log \frac{\lambda}{\lambda_{R}} & 0 & 0 \\
0 & 0 & \frac{1}{2} \log \frac{\lambda}{\lambda_{R}} & -\alpha \\
0 & 0 & -\alpha & \frac{1}{2} \log \frac{\lambda}{\lambda_{R}}
\end{array}\right)
$$

To compute the complexity, we need to decompose the generator $K^{(L R)}(4.29)$ in terms of the generators of $\operatorname{Sp}(4, R)$. The matrix generators for the $\operatorname{Sp}(4, R)$ can be split into the $\operatorname{Sp}(2, R)$ subalgebra acting on the left oscillator only

$$
T_{L, L}^{(1)}=\left(\begin{array}{cccc}
1 & 0 & 0 & 0 \\
0 & 0 & 0 & 0 \\
0 & 0 & -1 & 0 \\
0 & 0 & 0 & 0
\end{array}\right), \quad T_{L, L}^{(2)}=\left(\begin{array}{cccc}
0 & 0 & 0 & 0 \\
0 & 0 & 0 & 0 \\
-\sqrt{2} & 0 & 0 & 0 \\
0 & 0 & 0 & 0
\end{array}\right), \quad T_{L, L}^{(3)}=\left(\begin{array}{cccc}
0 & 0 & \sqrt{2} & 0 \\
0 & 0 & 0 & 0 \\
0 & 0 & 0 & 0 \\
0 & 0 & 0 & 0
\end{array}\right)
$$

the $\operatorname{Sp}(2, \mathrm{R})$ subalgebra acting on the right oscillator only

$$
T_{R, R}^{(1)}=\left(\begin{array}{cccc}
0 & 0 & 0 & 0 \\
0 & 1 & 0 & 0 \\
0 & 0 & 0 & 0 \\
0 & 0 & 0 & -1
\end{array}\right), \quad T_{R, R}^{(2)}=\left(\begin{array}{cccc}
0 & 0 & 0 & 0 \\
0 & 0 & 0 & 0 \\
0 & 0 & 0 & 0 \\
0 & -\sqrt{2} & 0 & 0
\end{array}\right), \quad T_{R, R}^{(3)}=\left(\begin{array}{cccc}
0 & 0 & 0 & 0 \\
0 & 0 & 0 & \sqrt{2} \\
0 & 0 & 0 & 0 \\
0 & 0 & 0 & 0
\end{array}\right)
$$

and the remaining generators which entangle the two oscillators

$$
\begin{array}{cc}
T_{L, R}^{(1)}=\left(\begin{array}{cccc}
0 & 0 & 0 & 0 \\
1 & 0 & 0 & 0 \\
0 & 0 & 0 & -1 \\
0 & 0 & 0 & 0
\end{array}\right), & T_{R, L}^{(1)}=\left(\begin{array}{cccc}
0 & 1 & 0 & 0 \\
0 & 0 & 0 & 0 \\
0 & 0 & 0 & 0 \\
0 & 0 & -1 & 0
\end{array}\right), \\
T_{L, R}^{(2)}=\left(\begin{array}{cccc}
0 & 0 & 0 & 0 \\
0 & 0 & 0 & 0 \\
0 & -1 & 0 & 0 \\
-1 & 0 & 0 & 0
\end{array}\right), & T_{L, R}^{(3)}=\left(\begin{array}{llll}
0 & 0 & 0 & 1 \\
0 & 0 & 1 & 0 \\
0 & 0 & 0 & 0 \\
0 & 0 & 0 & 0
\end{array}\right) .
\end{array}
$$


According to the above generators, $K^{(L R)}(4.29)$ can be written as follows

$$
K^{(L R)}=-\frac{1}{2} \log \frac{\lambda}{\lambda_{R}}\left(T_{L, L}^{(1)}+T_{R, R}^{(1)}\right)+\alpha\left(T_{L, R}^{(1)}+T_{R, L}^{(1)}\right),
$$

which it means that only four components of the tangent vector $Y^{I}$ are non-vanishing. The above result implies that the $F_{1}$ complexity becomes

$$
\mathcal{C}_{1}^{(L R)}=\left|Y_{L, L}^{(1)}\right|+\left|Y_{L, R}^{(1)}\right|+\left|Y_{R, L}^{(1)}\right|+\left|Y_{R, R}^{(1)}\right|=2|\alpha|+\left|\log \frac{\lambda}{\lambda_{R}}\right|,
$$

and by that, the complexity of formation is given by

$$
\mathcal{C}_{1}^{(L R)}-\mathcal{C}_{1}^{(L R)}(\operatorname{vac})=2|\alpha|,
$$

where it only depends on $\beta(\omega+\mu q)$ and it contains no information about the reference state. ${ }^{4}$ Accordingly, the contribution of all modes to the complexity of formation becomes

$$
\mathcal{C}_{1}^{(L R)}-\mathcal{C}_{1}^{(L R)}(\operatorname{vac})=\frac{\operatorname{vol} \Omega_{d-2}}{\beta^{d-1}(2 \pi)^{d-1}} \int_{0}^{\infty} d u u^{d-2} \log \left(\frac{1+e^{-\frac{1}{2}\left(\sqrt{u^{2}+s^{2}}+\tilde{Q}\right)}}{1-e^{-\frac{1}{2}\left(\sqrt{u^{2}+s^{2}}+\tilde{Q}\right)}}\right) .
$$

The ratio of the complexity of formation to thermal entropy is provided in figure 12 for different dimensions and different values of chemical potential. Intriguingly, this ratio is free of UV divergence but has a new IR divergence for $T \rightarrow 0$ case. Similarly, in the holographic counterpart, for the complexity of formation there is still a cancellation of the UV divergences associated with the asymptotic boundary and instead exists an IR divergence associated with the infinitely long throat of the extremal black holes. The above results suggests that preparing the 'extremal' thermofield double states at zero temperature and finite chemical potential are infinitely hard compared to the finite temperature states in complete agreement with third law of holographic complexity, [9]. To complete the study of complexity of formation in this basis, let us present the result for massive complex scalar. The effect of changing dimension and chemical potential for this case are presented figure 13. Both the complexity of formation and the entropy go to zero as the parameter $\beta m$ increases, but the ratio increases exponentially as a function of $\beta m$.

\subsection{Time dependency of complexity}

To evaluate the complexity growth rate of cTFD state in LR basis we need the full timedependent relative covariance matrix. Similar to previous analysis, let us firstly concentrate on a single mode. To obtain that matrix, we start with the covariance matrix itself in diagonal basis

$$
G^{( \pm)}(t)=G_{\mathrm{TFD}}^{+}(t) \oplus G_{\mathrm{TFD}}^{-}(t),
$$

where the direct sum inputs the + and - components in a 4 by 4 combined matrix and where the $G_{\mathrm{TFD}}^{+}(t)$ was defined in (3.29) by noting to $\alpha \rightarrow \alpha_{p}$ and $G_{\mathrm{TFD}}^{-}(t)$ is a same matrix just with $\alpha_{p} \rightarrow-\alpha_{p}$. According to the following definition

$$
G_{\mathrm{T},+}(t)=U_{+}(t) G_{R,+} U_{+}^{\mathrm{T}}(t),
$$

\footnotetext{
${ }^{4}$ It is worth noting that in (4.34) all gates are uniformly weighted.
} 

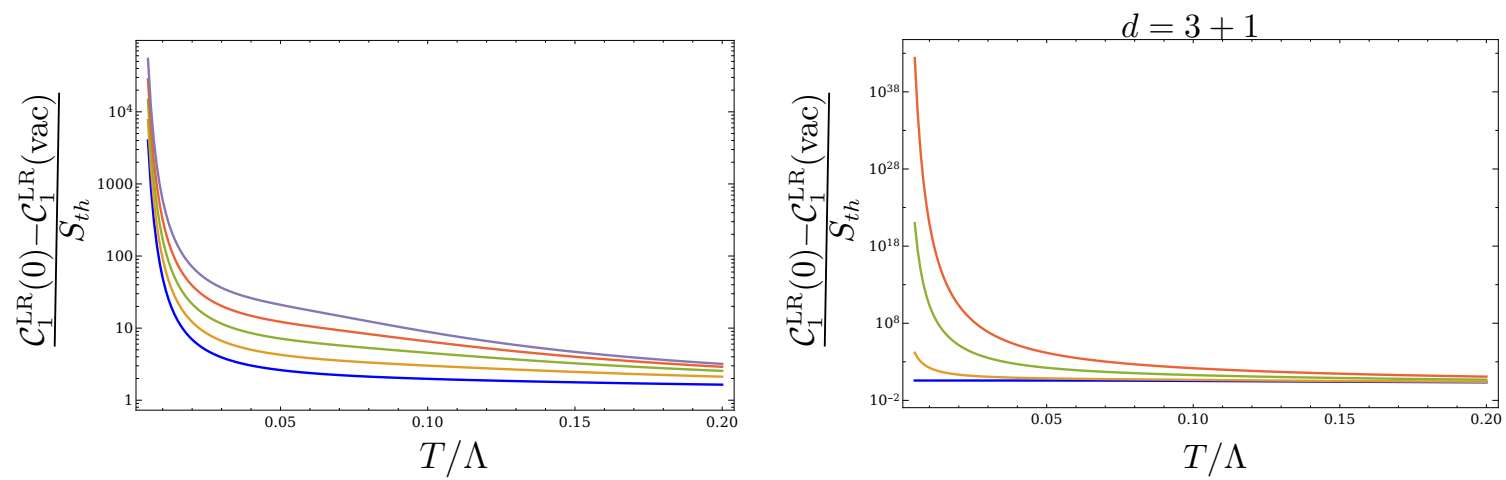

Figure 12. Left: $\mathcal{C}_{1}$ complexity of formation normalized by the entropy in $L R$ basis at constant chemical potential $\mu q=10^{-1} \Lambda$ and very small mass $m=10^{-5} \Lambda$ for $d=1+1$ (blue), $2+1$ (brown), $3+1$ (green), $4+1$ (red) and $5+1$ (purple). For small temperatures, the profile of the ratio of the complexity of formation over the entropy diverges. For higher temperatures, it decreases to a constant value which it depends to dimension. Right: $\mathcal{C}_{1}$ complexity of formation normalized by the entropy in $L R$ basis at different constant chemical potential $\mu q=0$ (blue), $\mu q=10^{-1} \Lambda$ (brown), $\mu q=\Lambda / 2$ (green) and $\mu q=\Lambda$ (red) with very small mass $m=10^{-5} \Lambda$. For small temperatures, the profile of the ratio of the complexity of formation over the entropy diverges. For higher temperatures, it decreases to a constant value.
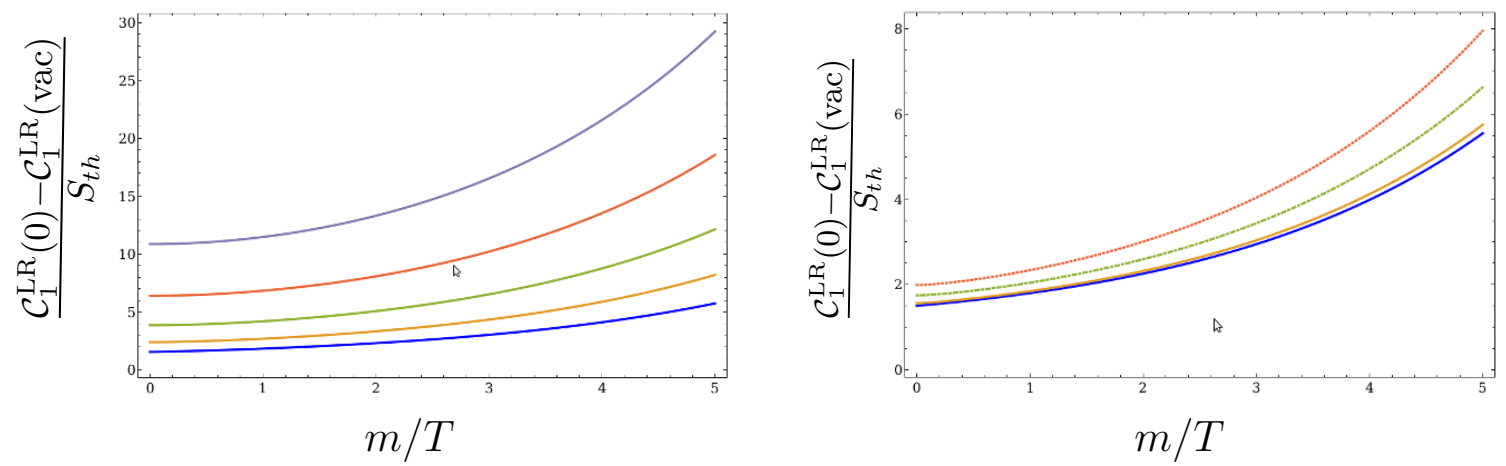

Figure 13. Left: The ratio of complexity of formation with thermal entropy in the LR basis for the cTFD state of a free complex scalar with a mass $m$ and $\tilde{Q}=10^{-1}$. We show the dependence on the dimension from $d=2$ (bottom) to $d=6$ (top). Right: The complexity of formation in the LR basis for the cTFD state of a free complex scalar with a mass $m$ and different $\tilde{Q}=10^{-6}$ (blue), $10^{-1}$ (brown), $1 / 2$ (dot-dashed green) and 1 (dotted red) in $d=1+1$.

for $\lambda_{R}=1$, it is easy to see that the transfer matrix $U_{+}(t)$ can be written as

$$
U_{+}(t)=\left(\begin{array}{cc}
\cosh s_{1,+}-\sin s_{2,+} \sinh s_{1,+} & \cos s_{2,+} \sinh s_{1,+} \\
\cos s_{2,+} \sinh s_{1,+} & \cosh s_{1,+}+\sin s_{2,+} \sinh s_{1,+}
\end{array}\right),
$$

with

$$
\begin{aligned}
& s_{1,+}=\frac{1}{2} \cosh ^{-1}\left(\frac{1+\lambda_{p}^{2}}{2 \lambda_{p}} \cosh 2 \alpha_{p}+\frac{1-\lambda_{p}^{2}}{2 \lambda_{p}} \sinh 2 \alpha_{p} \cos \left[\left(\omega_{p}+\mu q\right) t\right]\right) \\
& s_{2,+}=\tan ^{-1}\left(\frac{1+\lambda_{p}^{2}}{2 \lambda_{p}} \cot \left[\left(\omega_{p}+\mu q\right) t\right]+\frac{1-\lambda_{p}^{2}}{2 \lambda_{p}} \operatorname{coth} 2 \alpha_{p} \csc \left[\left(\omega_{p}+\mu q\right) t\right]\right) .
\end{aligned}
$$


Clearly, the identity $U_{-}(t)=U_{+}(t)\left(\alpha_{p} \rightarrow-\alpha_{p}\right)$, implies that

$$
s_{i,-}=s_{i,+}\left(\alpha_{p} \rightarrow-\alpha_{p}\right) .
$$

The transfer matrix (4.40) by using the $\mathrm{SL}(2, p)$ generators (4.24) can be decomposed as following

$$
U_{+}(t)=\exp \left[\left(-s_{1,+} \sin s_{2,+}\right) T_{1,+}+\left(\frac{s_{1,+} \cos s_{2,+}}{\sqrt{2}}\right)\left(T_{2,+}-T_{3,+}\right)\right] .
$$

Therefore, the full circuit $U_{( \pm)}$becomes

$$
U_{ \pm}(t) \equiv \exp \left[M_{ \pm}(t)\right]=\exp \left[\left(\begin{array}{cccc}
-s_{1,+} \sin s_{2,+} & s_{1,+} \cos s_{2,+} & 0 & 0 \\
s_{1,+} \cos s_{2,+} & s_{1,+} \sin s_{2,+} & 0 & 0 \\
0 & 0 & -s_{1,-} \sin s_{2,-} & s_{1,-} \cos s_{2,-} \\
0 & 0 & s_{1,-} \cos s_{2,-} & s_{1,-} \sin s_{2,-}
\end{array}\right)\right] .
$$

Now, we can find relevant generator $M_{(L R)}(t)=R_{4}^{\mathrm{T}} M_{( \pm)} R_{4}$ in the LR basis by apply the transformation (4.21). It is easy to see that

$$
M_{(L R)}(t)=\frac{1}{2}\left(\begin{array}{cccc}
M_{11}(t) & M_{12}(t) & M_{13}(t) & M_{14}(t) \\
M_{12}(t) & M_{11}(t) & M_{14}(t) & M_{13}(t) \\
M_{13}(t) & M_{14}(t) & -M_{11}(t) & M_{34}(t) \\
M_{14}(t) & M_{13}(t) & M_{34}(t) & -M_{11}(t)
\end{array}\right),
$$

with

$$
\begin{array}{ll}
M_{11}(t)=-s_{1,-} \sin s_{2,-}-s_{1,+} \sin s_{2,+}, & M_{12}(t)=s_{1,-} \sin s_{2,-}-s_{1,+} \sin s_{2,+}, \\
M_{13}(t)=s_{1,-} \cos s_{2,-}+s_{1,+} \cos s_{2,+}, & M_{14}(t)=-s_{1,-} \cos s_{2,-}+s_{1,+} \cos s_{2,+}, \\
M_{34}(t)=-s_{1,-} \sin s_{2,-}+s_{1,+} \sin s_{2,+} &
\end{array}
$$

The matrix $M_{(L R)}(t)$ (4.44) can be decomposed in terms of the generators of $\operatorname{Sp}(4, R),(4.30)-(4.32)$ as follows

$$
\begin{aligned}
M_{L R}(t)= & a_{L, L}^{(1)}\left(T_{L, L}^{(1)}+T_{R, R}^{(1)}\right)+a_{L, R}^{(1)}\left(T_{L, R}^{(1)}+T_{R, L}^{(1)}\right)+a_{L, R}^{(2)}\left(T_{L, R}^{(2)}-T_{L, R}^{(3)}\right) \\
& +a_{L, L}^{(2)}\left(T_{L, L}^{(2)}+T_{R, R}^{(2)}-T_{L, L}^{(3)}-T_{R, R}^{(3)}\right),
\end{aligned}
$$

where

$$
\begin{array}{ll}
a_{L, L}^{(1)}=-\frac{1}{2}\left(s_{1,-} \sin s_{2,-}+s_{1,+} \sin s_{2,+}\right), & a_{L, L}^{(2)}=-\frac{1}{2 \sqrt{2}}\left(s_{1,-} \cos s_{2,-}+s_{1,+} \cos s_{2,+}\right), \\
a_{L, R}^{(1)}=\frac{1}{2}\left(s_{1,-} \sin s_{2,-}-s_{1,+} \sin s_{2,+}\right), & a_{L, R}^{(2)}=\frac{1}{2}\left(s_{1,-} \cos s_{2,-}-s_{1,+} \cos s_{2,+}\right),
\end{array}
$$

and

$$
\begin{aligned}
& s_{1, \pm}=\frac{1}{2} \cosh ^{-1}\left(\frac{1}{2 \lambda_{p}} \cosh 2 \alpha_{p} \pm \frac{1}{2 \lambda_{p}} \sinh 2 \alpha_{p} \cos \left[\left(\omega_{p}+\mu q\right) t\right]\right) \\
& s_{2, \pm}=\tan ^{-1}\left(\frac{1}{2 \lambda_{p}} \cot \left[\left(\omega_{p}+\mu q\right) t\right] \pm \frac{1}{2 \lambda_{p}} \operatorname{coth} 2 \alpha_{p} \csc \left[\left(\omega_{p}+\mu q\right) t\right]\right) .
\end{aligned}
$$


Now, using the $F_{1}$ cost function, (3.8), we arrive at

$$
\mathcal{C}_{1}(t)=2\left|a_{L, L}^{(1)}\right|+2\left|a_{L, R}^{(1)}\right|+2\left|a_{L, R}^{(2)}\right|+4\left|a_{L, L}^{(2)}\right| .
$$

Firstly, let us study (4.49) in simple limit where $\omega_{R}$ is much bigger than any other scale, i.e. $\lambda_{p} \rightarrow 0$. In this limit we have

$$
\mathcal{C}_{1}^{(L R)}(t)-\mathcal{C}_{1}^{(L R)}(\mathrm{vac})=\frac{\operatorname{vol}}{(2 \pi)^{d-1}} \int_{0}^{\infty} d^{d-1} p \log \left(\cosh 2 \alpha_{p}+\left|\cos \left(\omega_{p}+\mu q\right) t\right| \sinh 2 \alpha_{p}\right) .
$$

From the above equation, one may conclude that the complexity oscillates in time. But as it is clear from figure 14, a saturation happens at the order of inverse temperature. ${ }^{5}$ This fast saturation happens since oscillatory behavior for all modes quickly dephase and as a result, summing over them averages out at a time of the inverse temperature. Of course, this is very different than what we see for holographic complexity, i.e. where we see a linear growth at late times. The late time saturation for holographic CFTs is indeed related to their chaotic/fast-scrambling characteristic. The complexity growth rate differs more with holography since it is negative at first and it becomes more negative by increasing the $\mathrm{U}(1)$ global charge, see figure 15 . The reason for this negativity can be easily understood from (4.50) where at $t=0$ the contribution of all of individual modes take their maximum value and after this time all oscillatory terms become misaligned and therefore the complexity begins to decrease.

It will be instructive if we also study the effect of global charge in the presence of mass. According to figures 16 and 17, if the mass or chemical potential are larger than the temperature then complexity oscillates with damping amplitude. This oscillatory behavior is clear from (4.50) and at late times we observe again a saturation to a constant value. To close this section, in the following, we explore the effect of the reference scale on the time evolution of complexity of cTFD state by changing the dimensionless parameter $\tilde{\gamma}$. It is clear from figure 18 that the decreasing of the complexity of formation to the minus values is an artifact of choosing the special value for reference scale and indeed it can grow very fast to positive values for intermediate scales, $\tilde{\gamma} \sim 1$. Of course, despite increasing at first for intermediate scale, it does not continue increasing for long times and it saturates fast. It is worth to emphasize that for both large and small values of $\tilde{\gamma}$ we see the same behavior, i.e. decreasing at first then saturating to a constant value. The constant value of saturation also depends on the value of $\tilde{\gamma}$, see figures 18-19. Moreover, it is worth noting that the dependency of complexity to reference scale at early transient times is the same as holographic results. Indeed, the time dependency of complexity for cTFD state dual to a charged eternal black hole exhibits non-universal behavior at early times due to the normalization of the null normals to boundary of WDW patch in the CA proposal which it is remained even after recovering affine parametrization. To be more precise, even after fixing reparametrization invariance by a boundary counterterm, the transient behavior is controlled by an arbitrary dimensionless parameter $l_{\mathrm{ct}} / l$ which it can be dual to $\omega_{R}$ in the

\footnotetext{
${ }^{5}$ Our result is in contradiction with previous attempts [33] to study complexity of cTFD state of free complex scalar theory, since the authors find that the complexity grows linearly for a long time.
} 

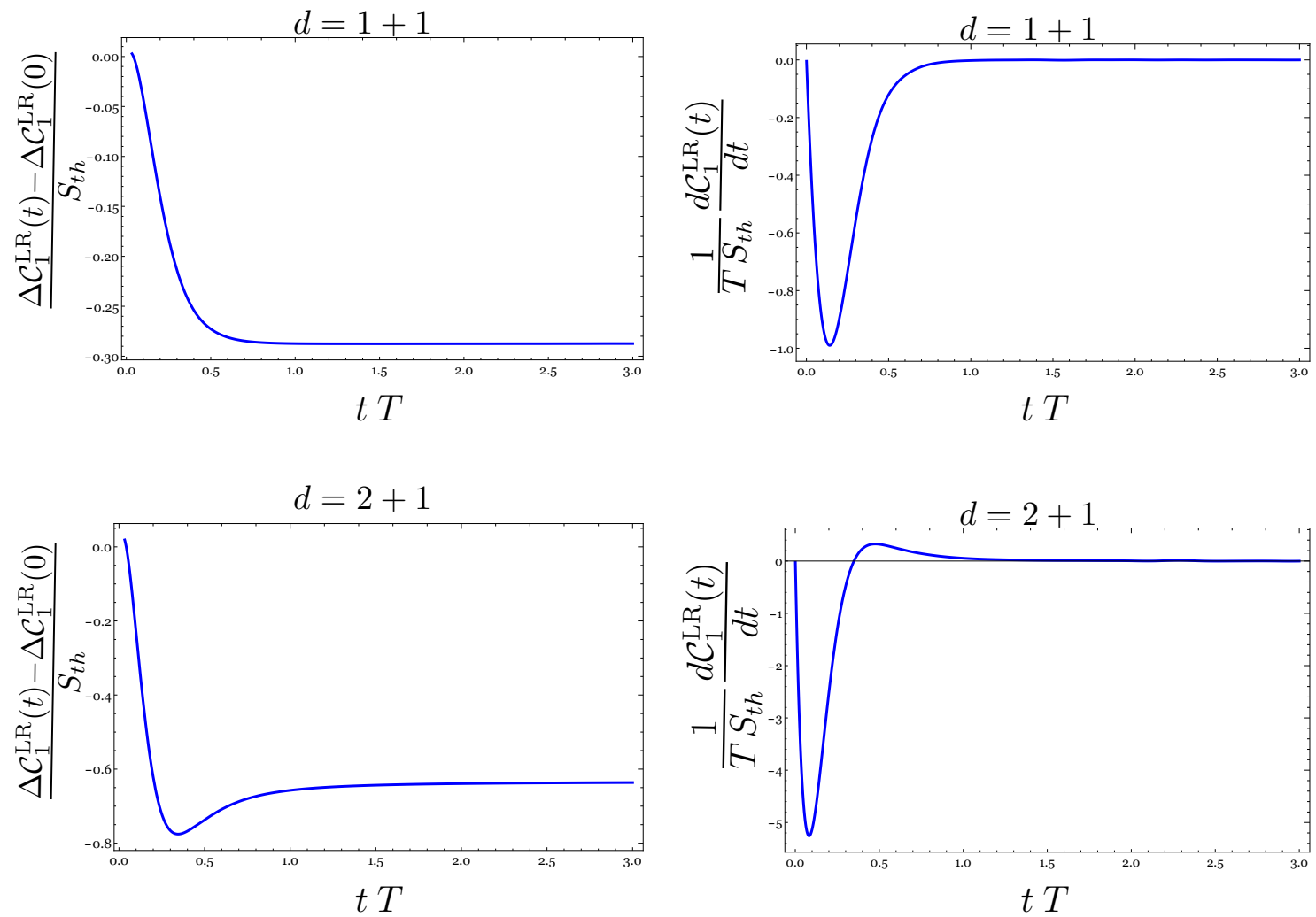

Figure 14. The $L^{1}$ norm complexity and its time derivative for a cTFD state of a massless complex scalar field with $\tilde{Q}=1 / 10$ in the LR basis and simple limit $\left(\lambda_{p} \rightarrow 0\right) . d=1+1(u p)$ and $d=2+1$ (down). In contrast to holography, the rate of change is negative at first, then saturates to zero at times of the order of the inverse temperature.
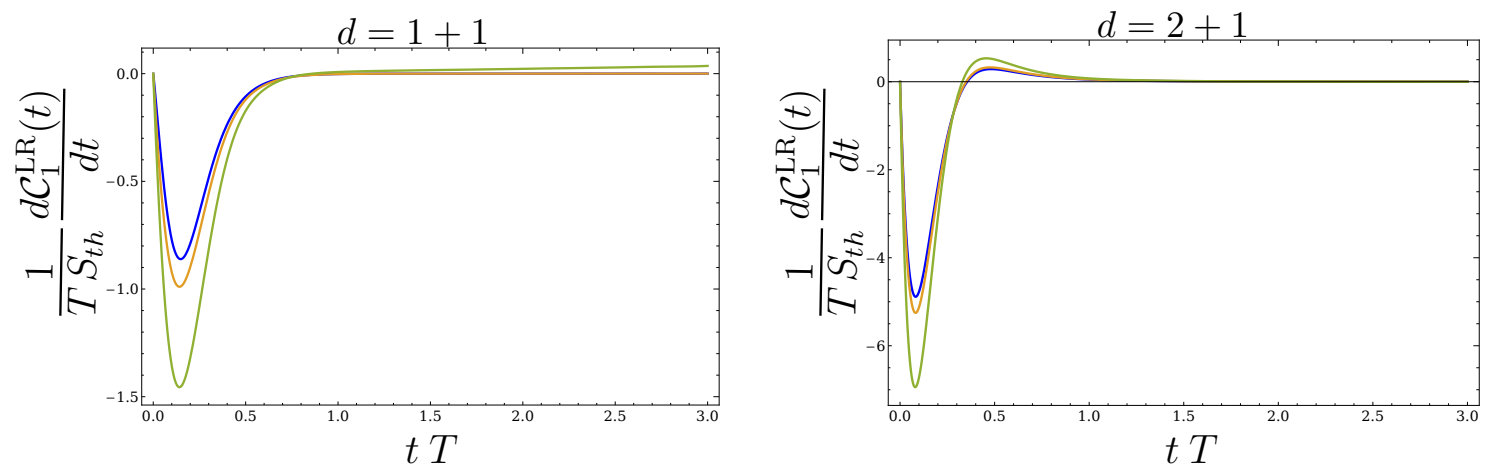

Figure 15. The $F_{1}$ complexity growth rate over entropy times temperature for cTFD state of a masless complex scalar with different chemical potential in $d=1+1$ (left) and $d=2+1$ (right). $\tilde{Q}=10^{-6}$ (blue), $\tilde{Q}=10^{-1}$ (brown) and $\tilde{Q}=1 / 2$ (green). 

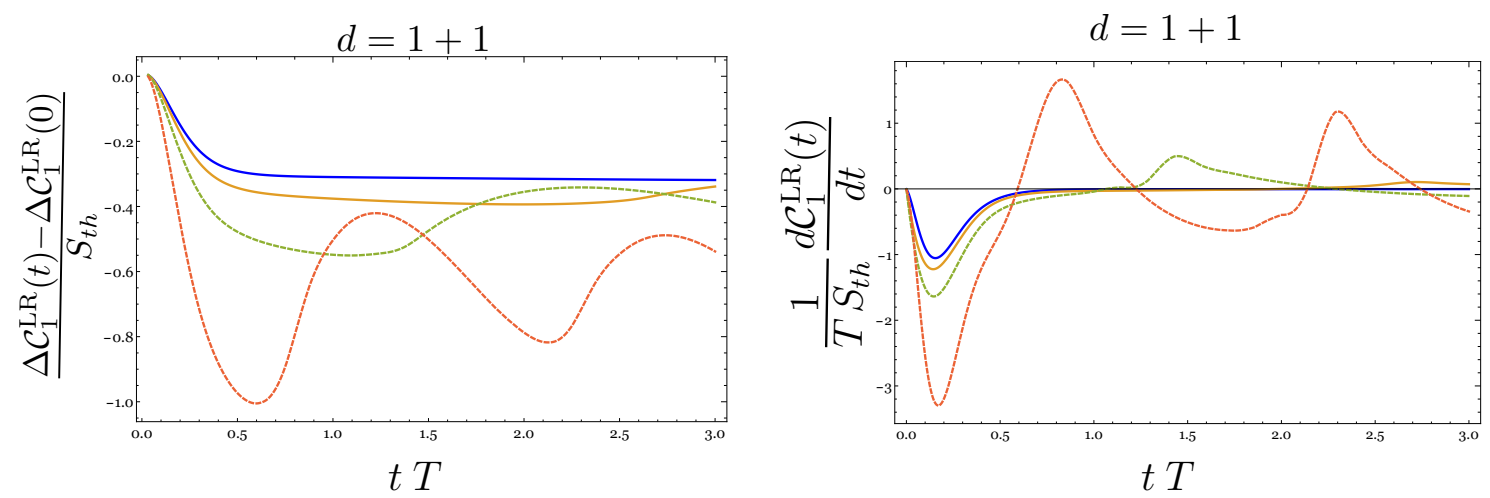

Figure 16. The $L^{1}$ norm complexity and its time derivative in the LR basis for a massive cTFD state with $\tilde{Q}=1 / 10$ in the simple limit $\left(\lambda_{p} \rightarrow 0\right) . \tilde{m}=1 / 5$ (blue), $\tilde{m}=1 / 2$ (brown), $\tilde{m}=1$ (dashed green) and $\tilde{m}=2$ (dashed red). For large masses with respect to the thermal scale, there is an oscillatory behavior with period $\Delta t \sim \pi /(m+\mu q)$.
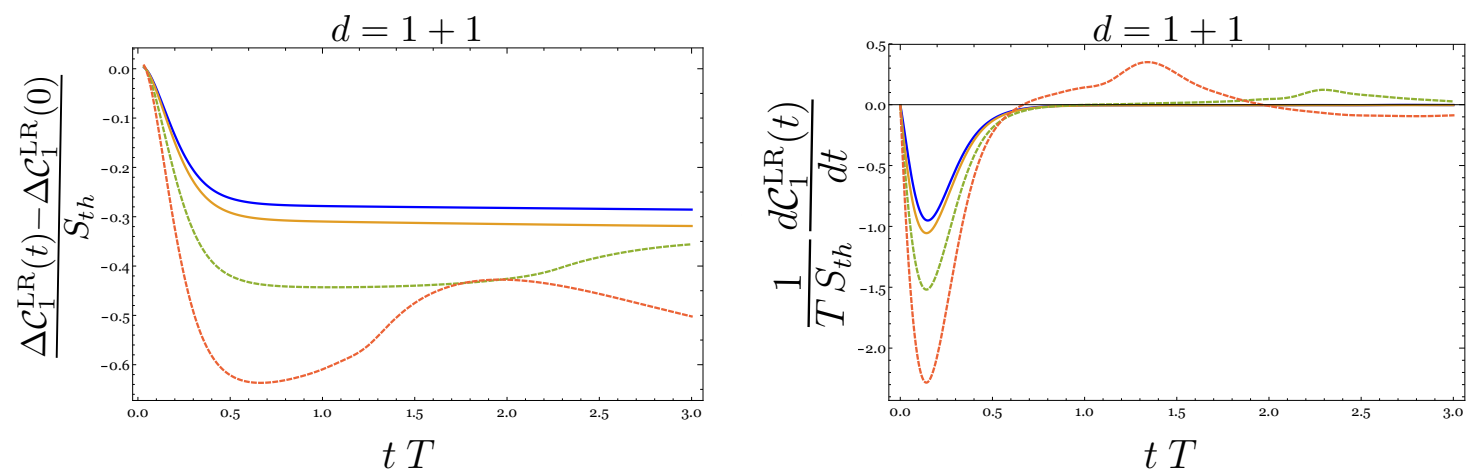

Figure 17. The $L^{1}$ norm complexity and its time derivative in the LR basis for a massive cTFD state with $\tilde{m}=1 / 5$ in the simple limit $\left(\lambda_{p} \rightarrow 0\right) . \tilde{Q}=10^{-6}$ (blue), $\tilde{Q}=10^{-1}$ (brown), $\tilde{Q}=1 / 2$ (dashed green) and $\tilde{Q}=1$ (dashed red). For large chemical potentials with respect to the thermal scale, there is an oscillatory behavior with period $\Delta t \sim \pi /(m+\mu q)$.

field theory side. The late time growth rate of complexity in holography was independent of these ambiguities in the null boundaries which also seems to be a property in figures 19 . Similar analysis for the massive case is presented in figures 20 and 21. Same as the massless case, for the large and small values of $\tilde{\gamma}$ we see effectively the same behavior. This can be understood easily by finding the complexity of formation in $L R$ bases for the simple limit in which $\omega_{R}$ is much smaller than any other scale, i.e. $\lambda_{p} \rightarrow \infty$. In this limit, using (4.40), we have

$$
\begin{aligned}
& s_{1, \pm}=\frac{1}{2} \log \left(\cosh 2 \alpha_{p} \mp \sinh 2 \alpha_{p} \cos \left[\left(\omega_{p}+\mu q\right) t\right]\right)+\frac{1}{2} \log \lambda_{p}, \\
& s_{2, \pm} \simeq \mp \operatorname{sgn}\left(\sin \left(\omega_{p}+\mu q\right) t\right) \frac{\pi}{2} .
\end{aligned}
$$



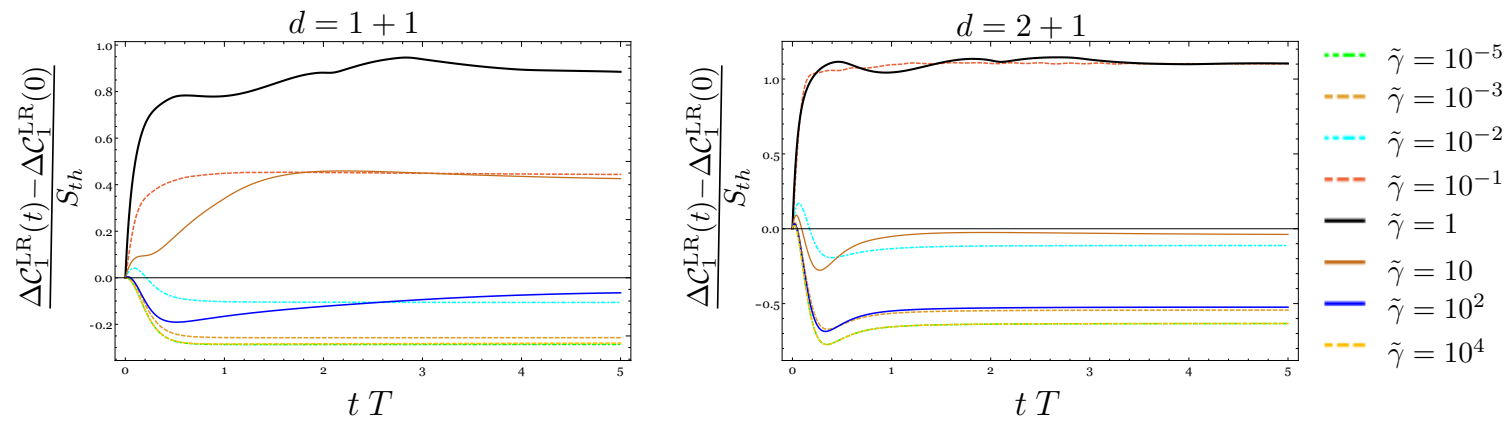

Figure 18. The time evolution of $F_{1}$ complexity normalized by thermal entropy with varying reference scale for the massless complex scalar with $\tilde{Q}=1 / 10 . \quad d=1+1$ (left) and $d=2+1$ (right).
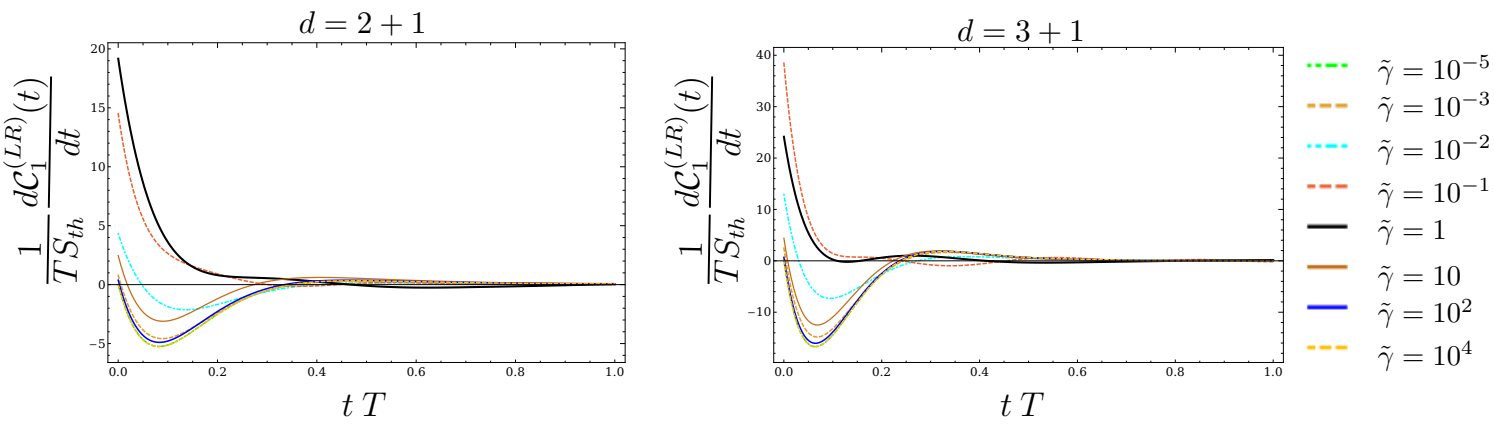

Figure 19. The $L^{1}$ norm complexity growth rate over temperature times thermal entropy for a cTFD state of massless complex scalar with $\tilde{Q}=1 / 10$ and different values of reference state scale. $d=2+1$ (left), $d=3+1$ (right).

Considering the above values for $s_{i, \pm}$ in (4.47) implies that

$$
\begin{array}{lll}
a_{L, L}^{(1)}=-\frac{1}{2}\left(s_{1,-} \sin s_{2,-}+s_{1,+} \sin s_{2,+}\right), & a_{L, R}^{(1)}=\frac{1}{2}\left(s_{1,-} \sin s_{2,-}-s_{1,+} \sin s_{2,+}\right), \\
a_{L, R}^{(2)} \simeq 0, & a_{L, L}^{(2)} \simeq 0,
\end{array}
$$

which by implementing them in (4.49) we have

$$
\mathcal{C}_{1}^{(L R)}(t)-\mathcal{C}_{1}^{(L R)}(\mathrm{vac})=\frac{\operatorname{vol}}{(2 \pi)^{d-1}} \int_{0}^{\infty} d^{d-1} p \log \left(\cosh 2 \alpha_{p}+\left|\cos \left(\omega_{p}+\mu q\right) t\right| \sinh 2 \alpha_{p}\right) .
$$

The result (4.53) exactly matches with (4.50). Thus, even though we are considering the opposite limit here (i.e. $\lambda \rightarrow \infty$ rather than $\lambda \rightarrow 0$ ), the time dependency of complexity of formation remains unchanged.

\section{Conclusions}

In this paper, we derived the complexity of formation over entropy for cTFD state of free complex scalar theory with particles on one side and anti-particles on the other side. We chose Nielsen's geometric approach and used covariance matrix technique, since our states 

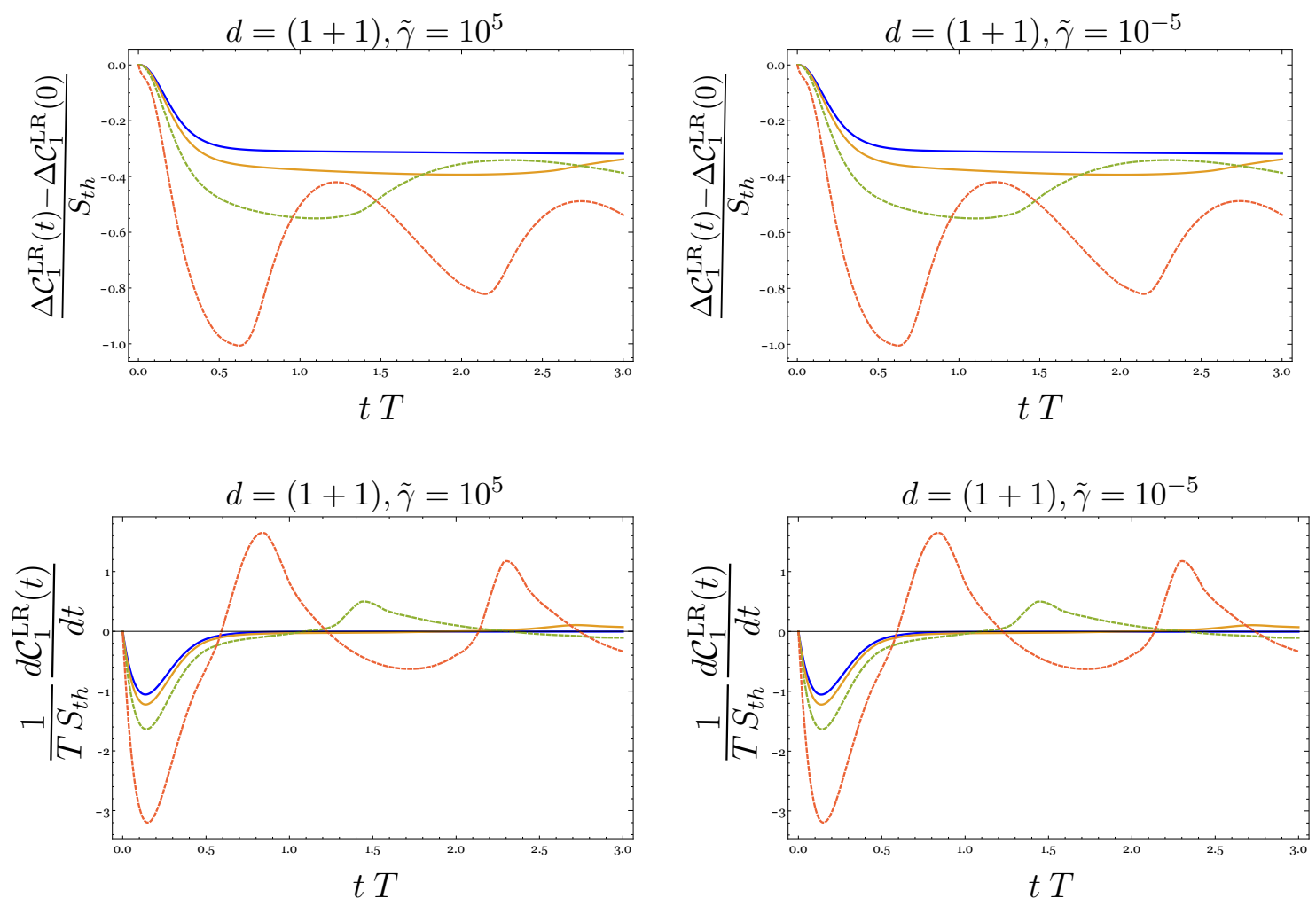

Figure 20. The time evolution of $L^{1}$ norm complexity of cTFD state in LR basis with $\tilde{Q}=1 / 10$ in $d=1+1$ dimensions and different masses. $\tilde{m}=1 / 5$ (blue), $\tilde{m}=1 / 2$ (brown), $\tilde{m}=1$ (dashed green) and $\tilde{m}=2$ (dashed red). The left figure is for $\tilde{\gamma}=10^{5}$ and the right one for $\tilde{\gamma}=10^{-5}$. It is clear that both the large and small values of reference scale led to the same time dependency for the complexity.

were Gaussian. We investigated $F_{\kappa=2}, F_{2}$ and $F_{1}$ cost functions to see which one is a better option to be the quantum field counterpart of holographic complexity. In general, $L^{2}$ norms are not basis-dependent and it makes them easier to work with. But their leading divergent term for $F_{2}$ and $F_{\kappa=2}$ cost functions are in conflict with the holographic results (4.15). On the other hand, if we take $\tilde{\alpha}=l \omega_{R}, \mathcal{C}_{1}$ would have the same leading term as holography. So after this observation, we mainly focused on $F_{1}$. As a matter of fact, we could just find an upper bound for this cost function and calculating the exact expression for $\mathcal{C}_{1}$ is very difficult. Also, this cost function is basis-dependent so we had to choose our basis. In the beginning, we used diagonal basis in which we can factorize our cTFD state. $\mathcal{C}_{1}$ in diagonal basis was in contrast with the third law of complexity, even though it had the correct leading terms. So we changed our basis to LR which uses physical degrees of freedom and it gave us the desired IR divergence that we see in holography.

Then we investigated the time dependency of complexity and it's growth rate, using $F_{1}$ in LR basis. A difference with holographic complexity is saturation time, which is much less than the expected one in holography, because we are using free field theory so our state cannot probe a vast subspace of Hilbert space and complexity saturates in thermal time. Besides, we observed that the reference scale has the same rule that the ambiguities 

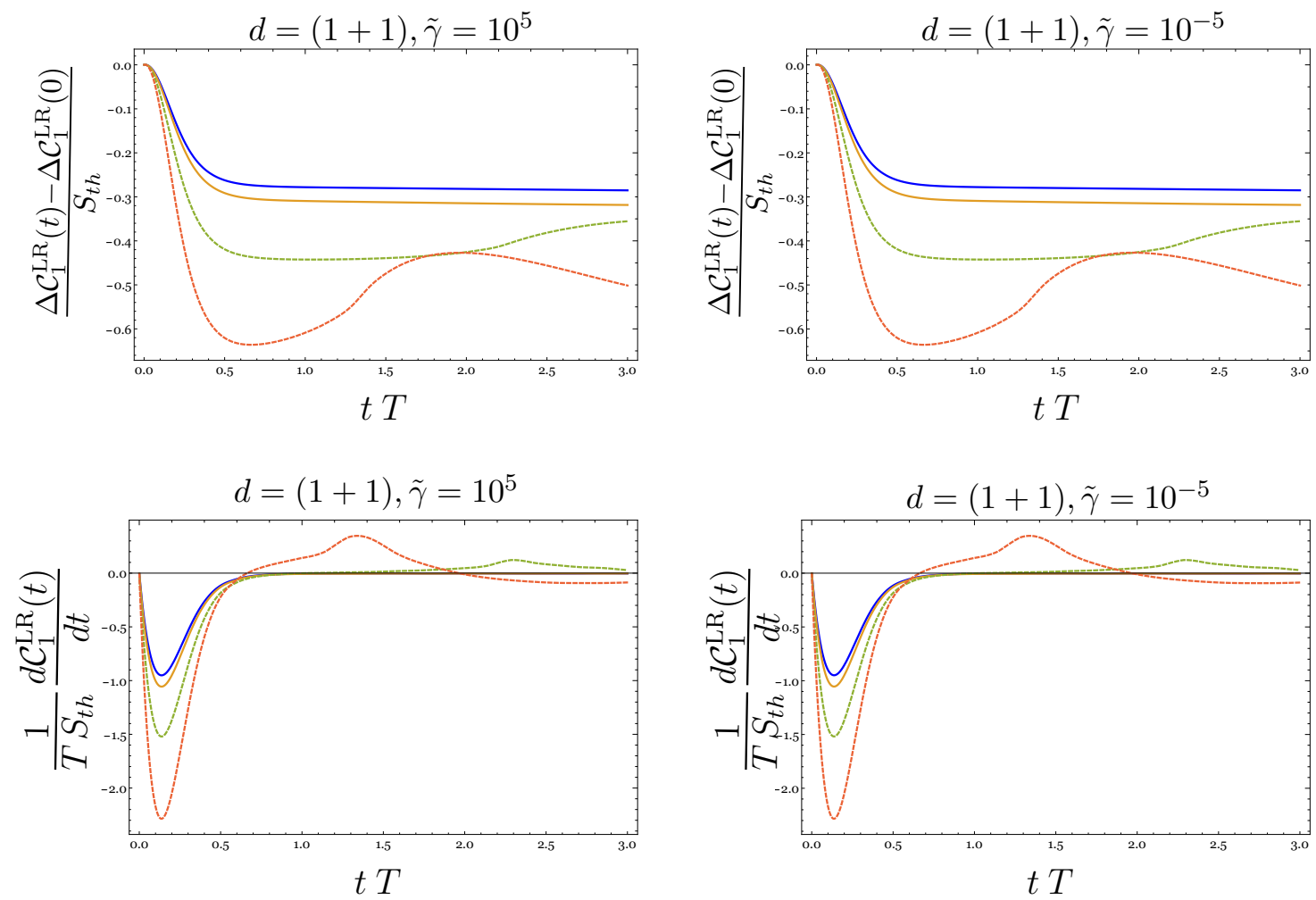

Figure 21. The time evolution of $L^{1}$ norm complexity of cTFD state in LR basis with $\tilde{m}=1 / 5$ in $d=1+1$ dimensions and different charges. $\tilde{Q}=10^{-6}$ (blue), $\tilde{Q}=10^{-1}$ (brown), $\tilde{Q}=1 / 2$ (dashed green) and $\tilde{Q}=1$ (dashed red). The left figure is for $\tilde{\gamma}=10^{5}$ and the right one is for $\tilde{\gamma}=10^{-5}$. It is clear that both the large and small values of reference scale led to the same time dependency for the complexity.

have in holography. For example, the dependency of complexity to reference scale at early transient times matches the holographic dependence on ambiguity.

Our final observation regarding complexity was comparing each term of it with different holographic proposals. We derived complexity of formation in holography for different dimensions. In $3+1$ dimensions, even though the neutral TFD was in agreement with holography, complexity of charged black hole had some terms missing. We discussed different ways to solve this problem. If we assume that the complexity we found in free cTFD does not change in strong coupling regime, we concluded that the most reasonable solution is adding boundary terms. One of these boundary terms were proposed in [34]. This term just recovers one of the missing terms and the remaining are still missing. But the other boundary term, proposed in [36] has all the missing terms and unlike the other one, smoothly approaches to neutral case. Moreover, in the finite chemical potential, the holographic complexity with the latter boundary term diverges similarly to the complexity of formation in cTFD.

So $\mathcal{C}_{1}$ in LR basis not only is a very good candidate for neutral black hole, but also it is a very good match in the presence of $\mathrm{U}(1)$ electric charge and complexity of formation in this basis matches very well with the theory that has cut off behind the horizon. 


\section{Acknowledgments}

We wish to thank Mohsen Alishahiha, Mostafa Ghasemi, S.Sedighe Hashemi, Ghadir Jafari, Ali Mollabashi, Behrad Taghavi, Farid Taghinavaz and Hamed Zolfi for fruitful discussions. We would also want to thank Michal Heller for useful comments on the early version of the paper and Shira Chapman for discussions and fruitful comments on the final draft. We especially would like to thank the IPM-Grid computing group for providing computing and storage facilities.

\section{A Thermal entropy and total charge}

Entropy of any thermal state can be obtained by its partition function, using the equation

$$
S_{t h}=\frac{\partial}{\partial T}(T \log Z)=\log Z-\beta \frac{\partial}{\partial \beta} \log Z .
$$

For a single mode, the partition function becomes

$$
Z=\frac{e^{-\beta(\omega+\mu q) / 2}}{1-e^{-\beta(\omega+\mu q)}},
$$

which it implies that the thermal entropy is given by

$$
S_{t h}=\operatorname{vol} \int \frac{\mathrm{d}^{d-1} k}{(2 \pi)^{d-1}}\left[\frac{\beta\left(\omega_{k}+\mu q\right)}{e^{\beta\left(\omega_{k}+\mu q\right)}-1}-\log \left(1-e^{-\beta\left(\omega_{k}+\mu q\right)}\right)\right] .
$$

Moreover, every particle has a charge $q$ so in order to find the total charge of the state, we need to find the average number of particles, using Bose-Einstein distribution. Accordingly,

$$
Q=q\left[\frac{1}{Z} \sum_{n=0}^{\infty} n e^{-\beta\left(\omega\left(n+\frac{1}{2}\right)+n \mu q\right)}\right]=\frac{q}{e^{\beta(\omega+\mu q)}-1}
$$

By integrating over every mode of the quantum field theory, we obtain

$$
Q_{\mathrm{tot}}=\operatorname{vol} \int \frac{\mathrm{d}^{d-1} k}{(2 \pi)^{d-1}} \frac{q}{e^{\beta\left(\omega_{k}+\mu q\right)}-1} .
$$

\section{B Complexities in diagonal basis}

In this appendix we explore the time dependency of $\mathcal{C}_{\kappa=2}$ (or it's $\mathcal{C}_{2}$ counterpart) and $\mathcal{C}_{1}$ complexities in diagonal basis. It is discussed in section 4 that these complexities are not consistent with holographic results neither for neutral AdS black holes nor for RNAdS ones. In figure 22, time evolution of those complexities is presented for the massless complex scalar theory. Although these complexities are basis independent but they are scale dependent. In contrast with $\mathcal{C}_{1}$ complexity in LR basis, they decrease by increasing the dimension. The difference of complexity at time " $\mathrm{t}$ " with complexity of TFD state at zero time is presented in figure 22 and the difference with the value for vacuum state is presented in figures 23-24. Moreover, for both of them the complexity increases sharply 

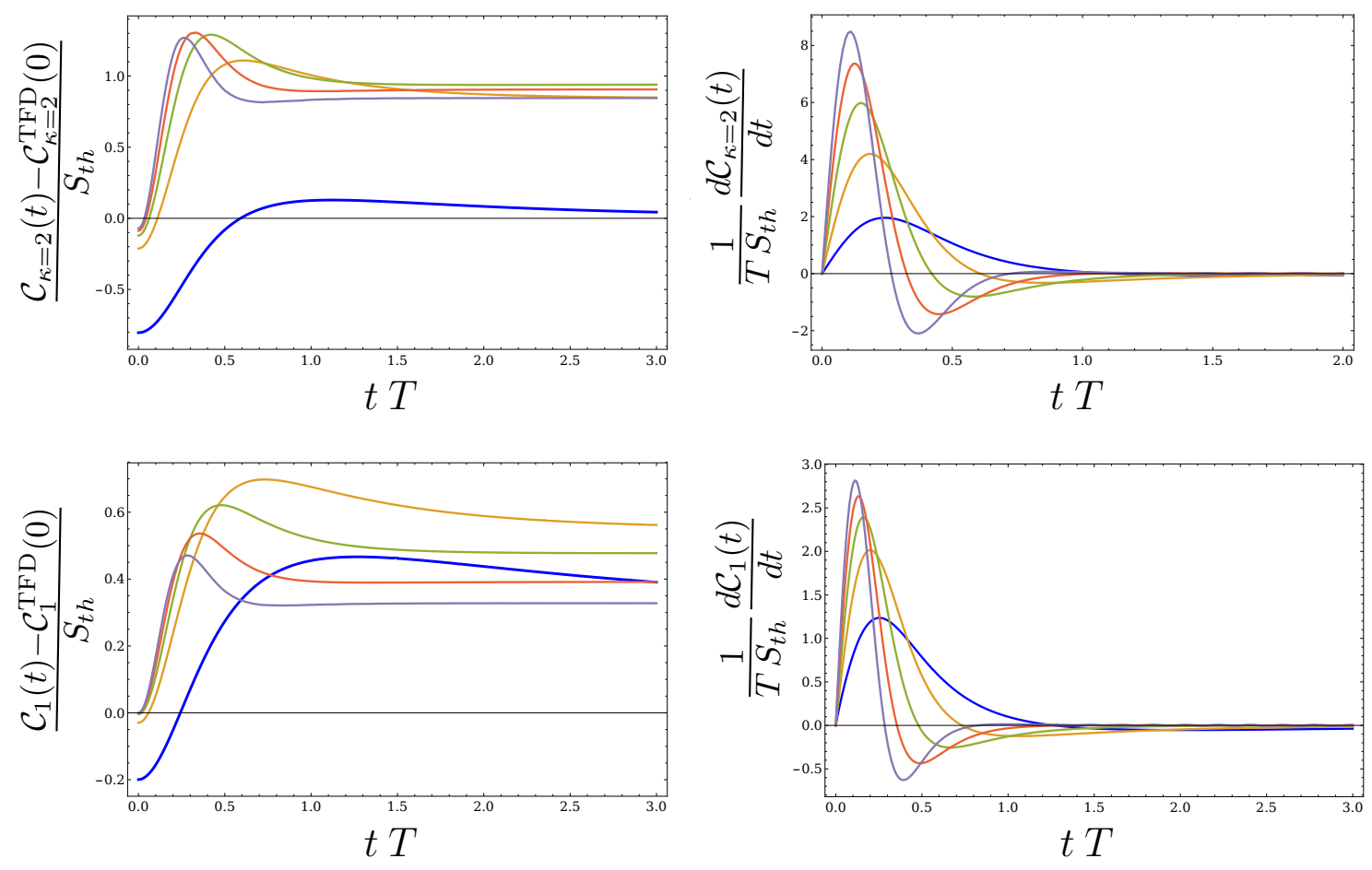

Figure 22. The time evolution of complexity of cTFD state in the diagonal basis for a massless complex scalar field with $\tilde{Q}=1 / 10$ in different dimensions. $d=2$ (blue), $d=3$ (brown), $d=4$ (green), $d=5$ (red) and $d=6$ (purple). The reference scale is $\tilde{\gamma}=10$. Up: $\kappa=2$, Down: $\kappa=1$.

at the beginning and reaches to its maximum value and then saturates very fast. This increasing in the beginning is another difference with $\mathcal{C}_{1}$ complexity in the LR basis but the fast saturation happens for both of the cases. The fast saturation is also in contrast with holographic results where one see a linear growth at late times. Since this linear growth is the effect of chaotic holographic CFTs this difference is not unexpected.

From figure 24, it is clear that the complexity growth rate for both $\mathcal{C}_{\kappa=2}$ and $\mathcal{C}_{1}$ measures is independent from the chemical potential which it is another difference with holographic proposals. Beside previously indicated reasons, this observation also implies that these complexities are not well suited to compare with holography. In figure 25, we study the effect of changing reference scale and moreover we see that sharply increasing value of complexity can become smooth by increasing value of $\tilde{Q}$. These complexities also saturate to different values by changing the reference scale and moreover for large and small $\tilde{\gamma}$ we have a large time derivative during the transient period at early times. This dependency to reference scale in early times is similar to the $\mathcal{C}_{1}$ complexity in LR basis and moreover is the same as holography and ambiguity in the normalization of null surfaces in CA conjecture. To complete the study, we do the same analysis for massive theory and results are presented in figures 26-27. Again we see a sharp increasing in the value of complexity but in contrast to massless case, for large masses (or large values of charges) with respect to the thermal scale there is an oscillatory behavior with period $\Delta t=\pi / \mathrm{m}$ (or $\Delta t=\pi / \mu q$ ). The complexity becomes smaller by increasing the mass or chemical 

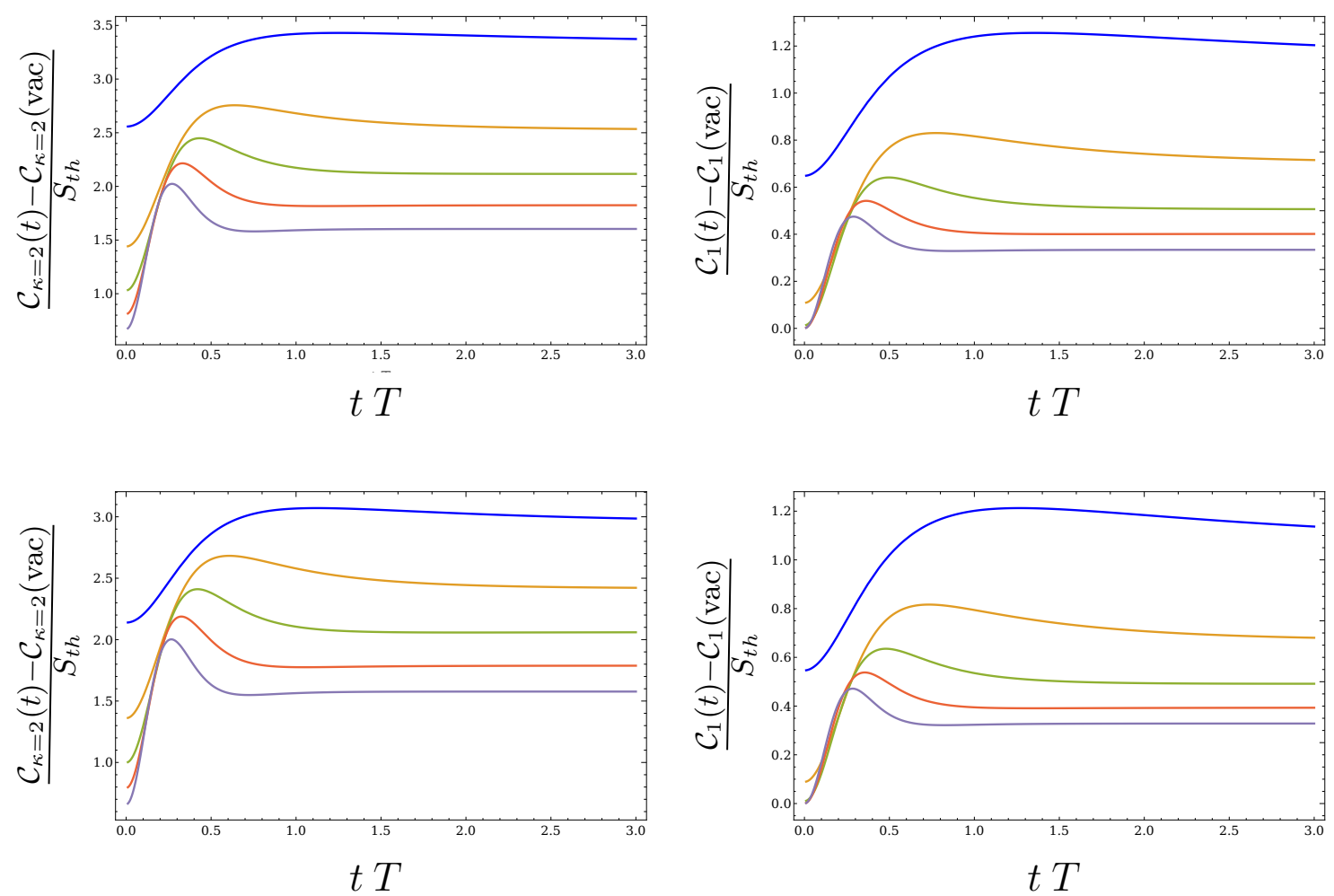

Figure 23. $U p$ : The time dependence of $\mathcal{C}_{\kappa=2}$ and $\mathcal{C}_{1}$ complexity of formation for TFD state of a massless real scalar theory with $\tilde{\gamma}=10$ in different dimensions. Down: The time dependence of $\mathcal{C}_{\kappa=2}$ and $\mathcal{C}_{1}$ complexity of formation for cTFD state of a massless complex scalar theory with $\tilde{Q}=1 / 10$ and $\tilde{\gamma}=10$ in different dimensions. $d=1+1$ (blue), $2+1$ (orange), $3+1$ (green) $4+1$ (red) and $5+1$ (purple).

potential and moreover at late times, we observe a saturation for complexity to a constant value. Albeit in all above cases we have chosen the UV cut-off as infinity, but complexity of formation is finite. This indicates that the UV divergences in the complexity of the timedependent cTFD state is exactly those of (two copies of) the vacuum not only in massless theory but also in massive one. Moreover, the UV divergences for the time-dependent cTFD state are the same with the ones for TFD state. This means that in presence of the U(1) global charge no new UV divergence appears which is similar to holography. In holographic dual, we do not need to add any new gravitational counterterm in presence of $\mathrm{U}(1)$ bulk field. The reason for the absence of new counterterms comes from the special fall of $U(1)$ gauge field near the boundary of spacetime.

The effect of changing the reference scale on time dependence of complexity is explored above, figure 25. Similarly, the results for time dependency of complexity of formation is presented in figure 28. Figures 25 and 28 show that by changing the reference scale, the general structure of time dependency of complexity of formation, which is increasing in the beginning and saturating after short time, in this basis does not change. This is in contrast with the result of $\mathcal{C}_{1}$ complexity in LR basis, figure 18, where by changing the reference scale, the decreasing of complexity in the beginning changes to increasing behavior and 

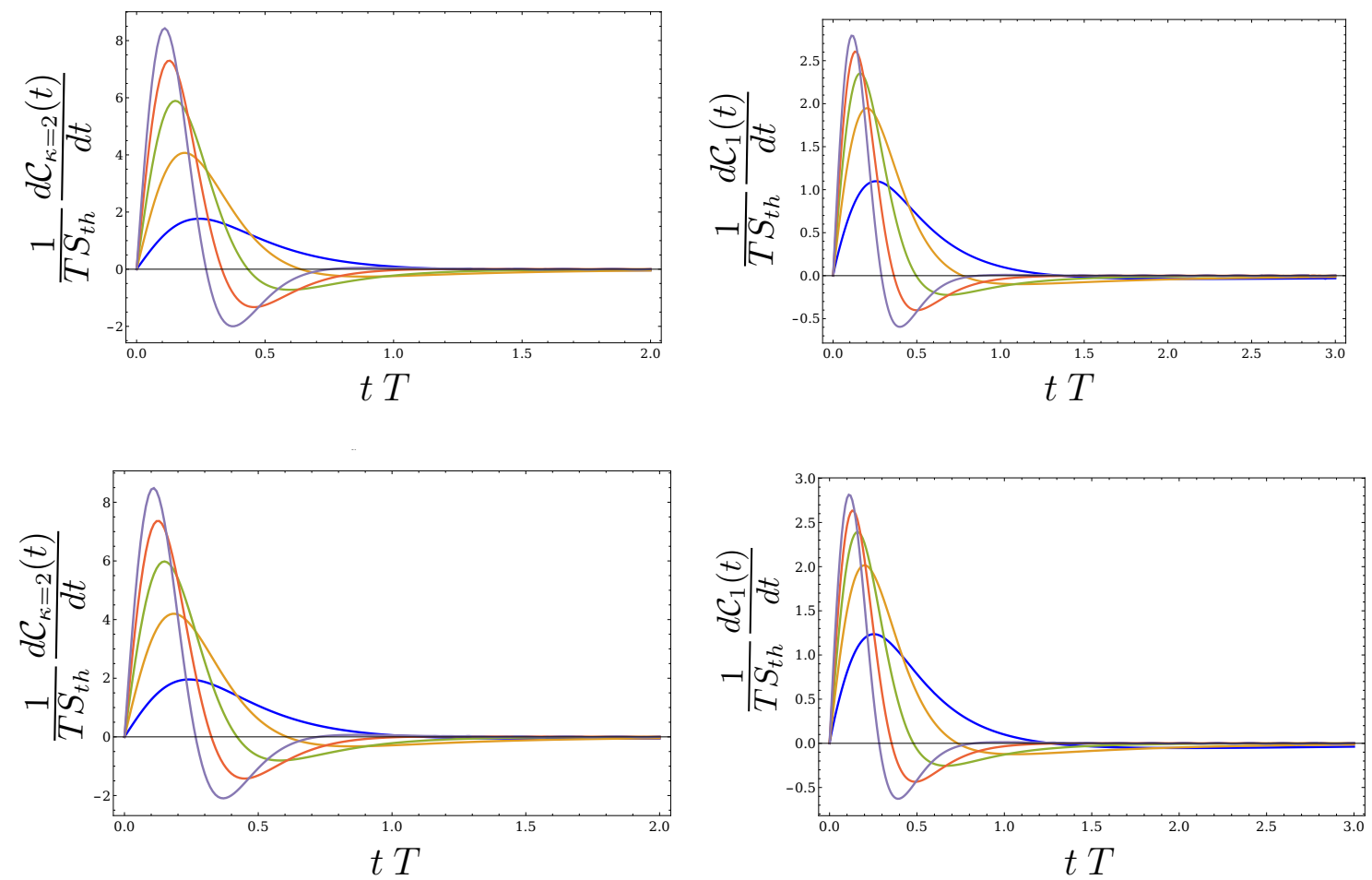

Figure 24. $U p$ : The $\mathcal{C}_{\kappa=2}$ and $\mathcal{C}_{1}$ complexity growth rate normalized by temperature times thermal entropy for TFD state of a massless real scalar theory with $\tilde{\gamma}=10$ in different dimensions. Down: The $\mathcal{C}_{\kappa=2}$ and $\mathcal{C}_{1}$ complexity growth rate normalized by temperature times thermal entropy for cTFD state of a massless complex scalar theory with $\tilde{Q}=1 / 10, \tilde{\gamma}=10$ in different dimensions. $d=1+1$ (blue), $2+1$ (orange), $3+1$ (green) $4+1$ (red) and $5+1$ (purple).

vice versa. But similar to the $\mathcal{C}_{1}$ in LR basis, for the large and small values of reference scale we see the same behavior. The effects of changing mass and charge on complexity of formation in this basis are provided respectively in figure 29 and figure 30.

To close this section, we explore the effect of changing the $\lambda_{R}$. Changing the ratio of the reference state scale to the gate scale i.e. $\lambda_{R}$ does not change the conclusions above significantly. The complexity still saturates at times of the order of $\beta$. From figure 31 we see that sharply changes of complexity becomes more and more smooth by increasing $\lambda_{R}$. This is consistent with this fact that by increasing $\lambda_{R}$, target state approaches to the reference state and therefore the complexity becomes smaller. For these norms the net effect of charge is just decreasing the value of complexity in each time without altering the general structure of graph specially its first time derivative. This is in contrast with the holographic results [9] which again, implies that these norms are not well suited to compare with large $N$ CFTs.

\section{More concrete comparison with holography}

To explore the relation between QFT calculations in subsection 4.1 with holographic results more concretely, it would be better to focus on the complexity of formation. The 

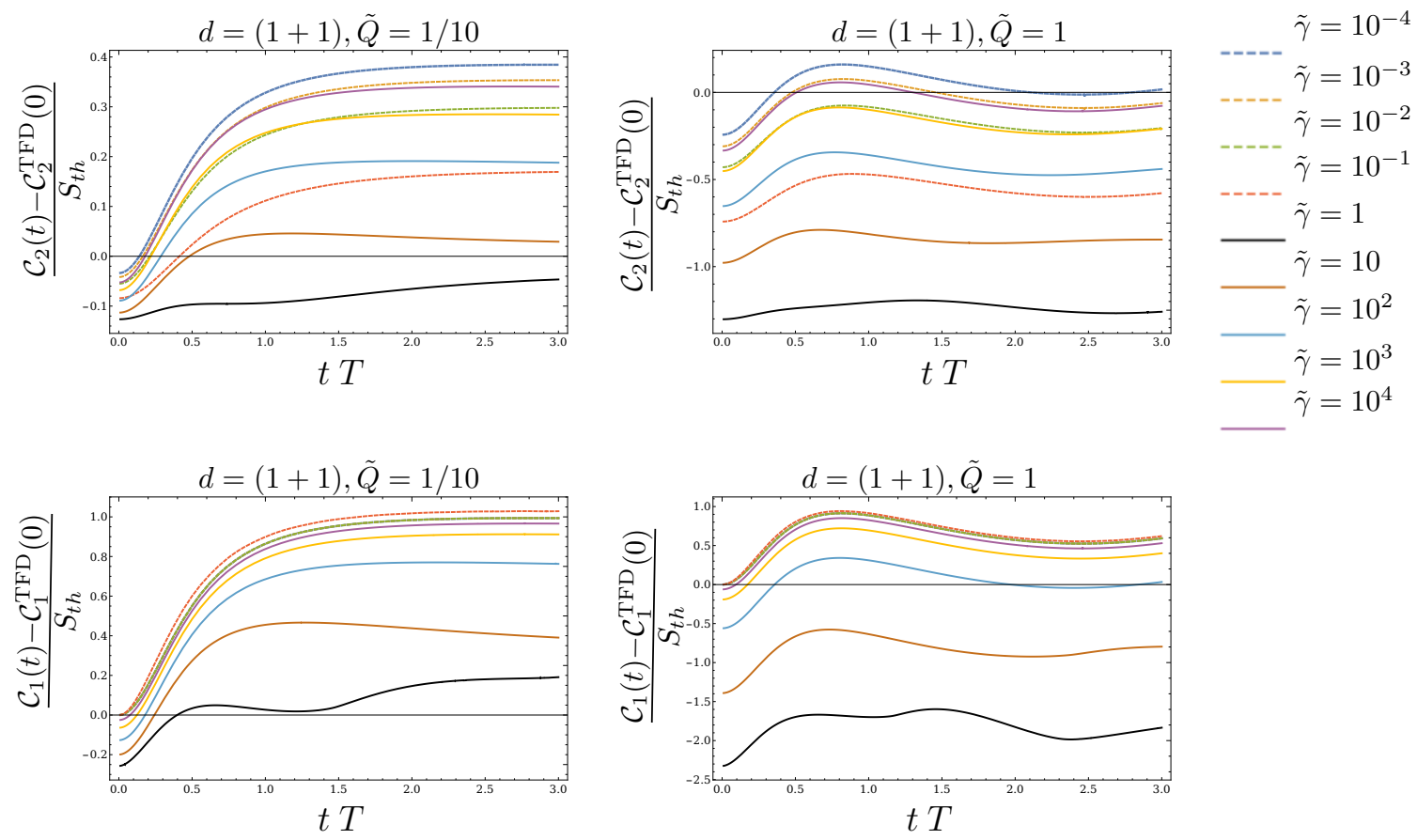

Figure 25. The time evolution of complexity with varying reference scale for massless complex scalar in $d=1+1$. $\tilde{\gamma}=1$ (solid black), $\tilde{\gamma}<1$ (dashed curves) and $\tilde{\gamma}>1$ (colored solid curves). For different values of $\tilde{\gamma}$ the complexity saturates to different constants at late times. We note that for large and small $\tilde{\gamma}$ we have a large time derivative during the transient period at early times. Increasing the charge actually smooth this transition. Left: $\tilde{Q}=1 / 10$, Right: $\tilde{Q}=1$, Up: $\mathcal{C}_{2}$ complexity and Down: $\mathcal{C}_{1}$ complexity.

$\mathcal{C}_{1}$ function (4.36) in LR basis together with (4.9) imply that the ratio of complexity of formation to thermal entropy for massless theory has the following expansion in $\tilde{Q}$,

$$
\frac{\mathcal{C}_{1}^{(L R)}-\mathcal{C}_{1}^{(L R)}(\mathrm{vac})}{S_{\mathrm{th}}}=\frac{2^{d}-1}{d}+\sum_{n=1}^{d-1} a(n, d) \tilde{Q}^{n}+b(d) \tilde{Q}^{d-1} \log \tilde{Q}+\mathcal{O}\left(\tilde{Q}^{d}\right)
$$

where $a(n, d)$ and $b(d)$ are constant numbers that depend on the dimensions, for example

$$
\begin{aligned}
& a(1,2)=\frac{6}{\pi^{2}}(1-\log 2), \quad a(1,3)=\frac{5 \pi^{2}}{54 \zeta(3)}, \quad a(1,4)=\frac{765 \zeta(3)}{8 \pi^{4}}, \\
& a(2,3)=\frac{1}{486 \zeta(3)^{2}}\left(5 \pi^{4}+27(-13+6 \log 2) \zeta(3)\right), \quad a(2,4)=-\frac{135}{16 \pi^{8}}\left(\pi^{6}-765 \zeta(3)^{2}\right), \\
& a(3,4)=-\frac{5}{32 \pi^{12}}\left(-211 \pi^{8}+48 \pi^{8} \log 2+5940 \pi^{6} \zeta(3)-2788425 \zeta(3)^{3}\right),
\end{aligned}
$$

and

$$
b(2)=-\frac{3}{2 \pi^{2}}, \quad b(3)=\frac{2}{9 \zeta(3)}, \quad b(4)=-\frac{165}{16 \pi^{4}} .
$$

According to CA proposal, to compute the complexity of cTFD state dual to charged eternal AdS-BH, one may find on-shell bulk action in a special part of geometry which is 

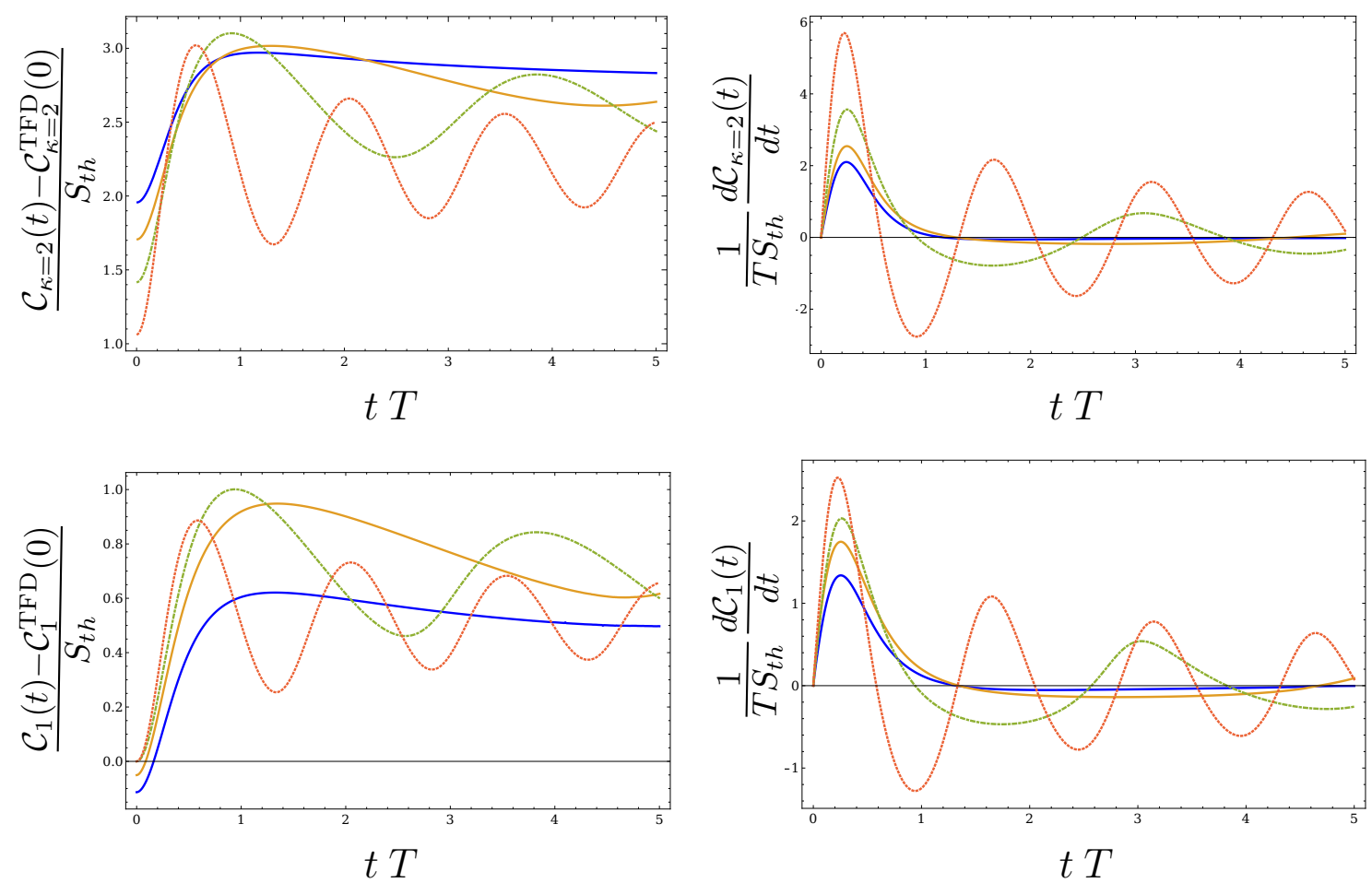

Figure 26. The time dependence of $\mathcal{C}_{\kappa=2}$ (up) and $\mathcal{C}_{1}$ (down) complexities for cTFD state of a massive complex scalar theory in $d=1+1$ dimensions with $\tilde{Q}=1 / 10, \tilde{\gamma}=10$ and different masses. $m=0.2 T$ (blue), $m=0.5 T$ (orange), $m=1 T$ (dotted dashed green) and $m=2 T$ (dotted red). For large masses with respect to the thermal scale, there is an oscillatory behavior with period $\Delta t=\pi / m$. At late times, we observe a saturation to a constant value.

known as WDW patch. The background geometry is

$$
d s^{2}=-f(r) d t^{2}+\frac{d r^{2}}{f(r)}+r^{2} d \Omega^{2}, \quad f(r)=\frac{r^{2}}{l^{2}}-\frac{\omega^{d-2}}{r^{d-2}}+\frac{q^{2}}{r^{2(d-2)}}
$$

and the WDW patch is shown as shaded region in left panel of figure 32. Temperature and entropy of this solution is given by

$$
T=\left.\frac{1}{4 \pi} \frac{\partial f}{\partial r}\right|_{r=r_{+}}, \quad S_{t h}=\frac{V_{d-1}}{4 G_{N}} r_{+}^{d-1}
$$

Furthermore, bulk gravitational action is as follows

$$
S_{\mathrm{Bulk}}=\frac{1}{16 \pi G_{N}} \int d^{d+1} x \sqrt{-G}(R-2 \Lambda)-\frac{1}{4 g^{2}} \int d^{d+1} x \sqrt{-G} F_{\mu \nu} F^{\mu \nu}
$$

To have an action which has well defined variational principle and its on-shell value also has reparamitrization invariance, one needs to add respectively Gibbons-Hawking term and proper counterterm. According to figure 32 for zero time at the boundary $\left(t_{L}=t_{R}=0\right)$, 

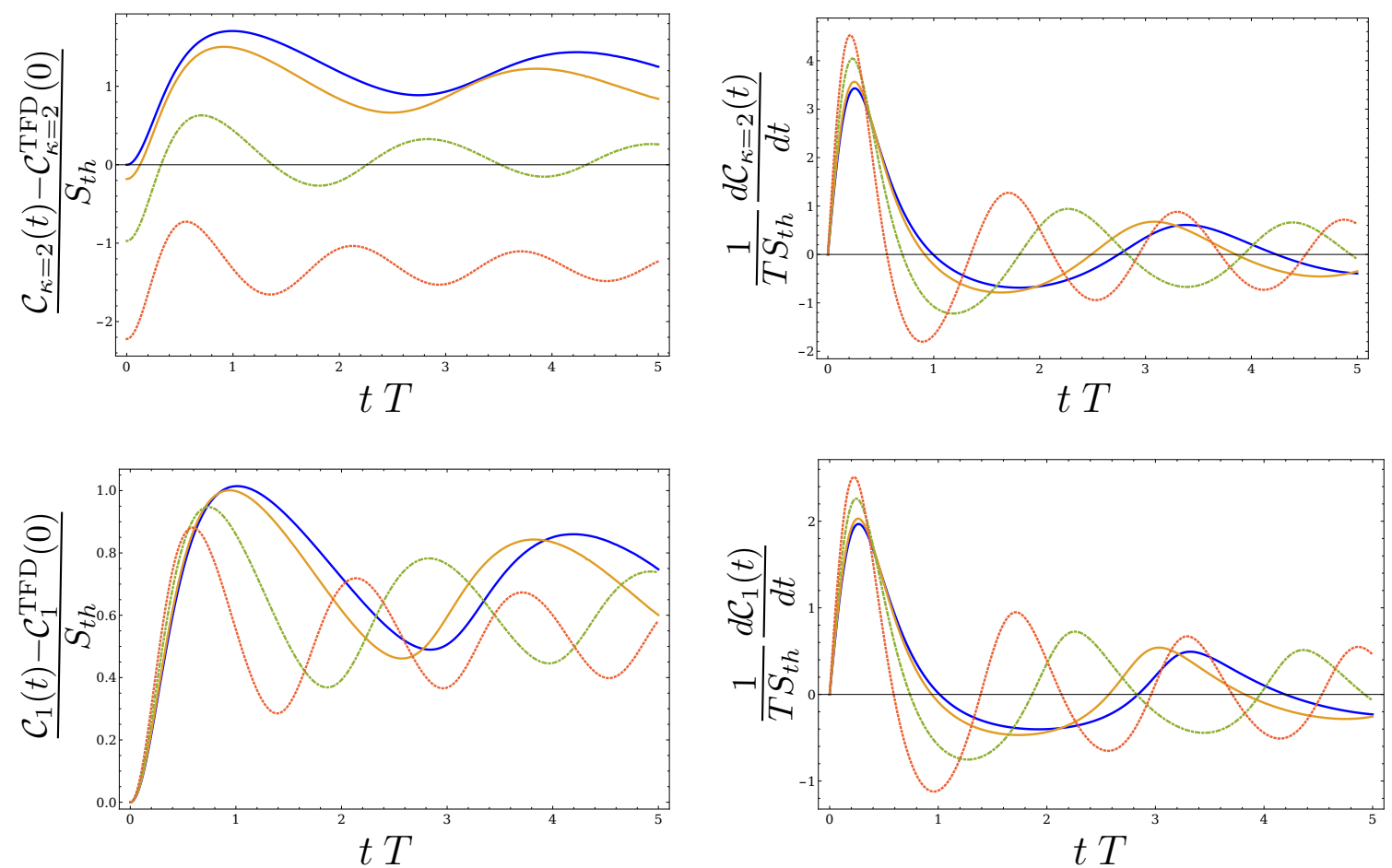

Figure 27. The time dependence of $\mathcal{C}_{\kappa=2}$ (up) and $\mathcal{C}_{1}$ (down) complexities for cTFD state of a massive complex scalar theory in $d=1+1$ dimensions with $\tilde{m}=1, \tilde{\gamma}=10$ and different charges. $\tilde{Q}=10^{-6}$ (blue), $\tilde{Q}=10^{-1}$ (orange), $\tilde{Q}=1 / 2$ (dotted dashed green) and $\tilde{Q}=1$ (dotted red). For large charges with respect to the thermal scale, there is an oscillatory behavior with period $\Delta t=\pi \beta / \tilde{Q}$. At late times, we observe a saturation to a constant value.
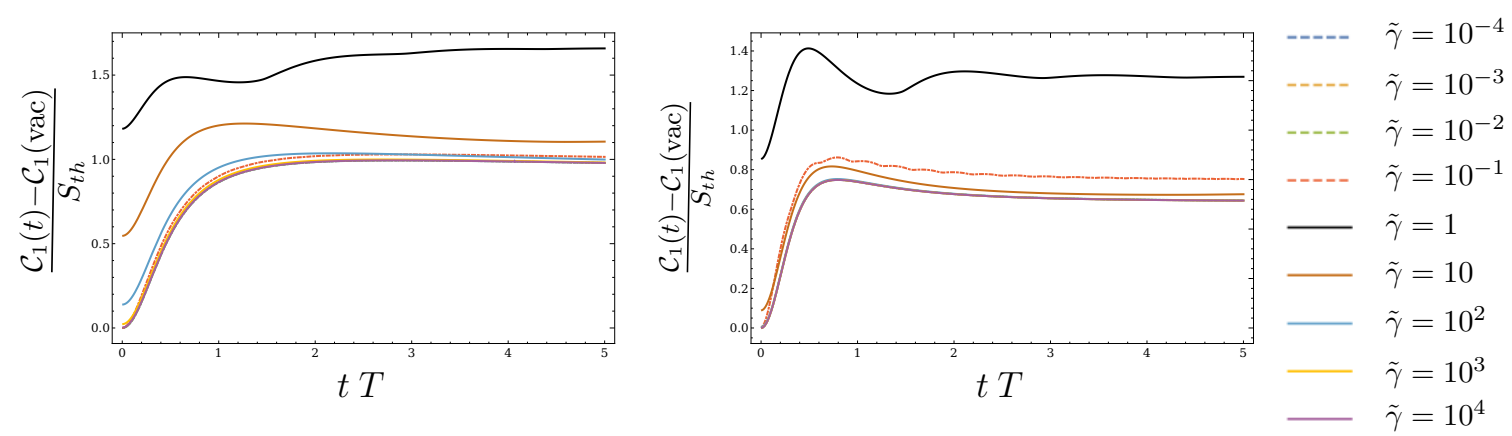

Figure 28. Time dependency of $\mathcal{C}_{1}$ complexity of formation in diagonal basis for a cTFD state of massless complex scalar theory with $\tilde{Q}=1 / 10$ and different values of reference scale. Left: $d=1+1$ and Right: $\mathrm{d}=2+1$. 

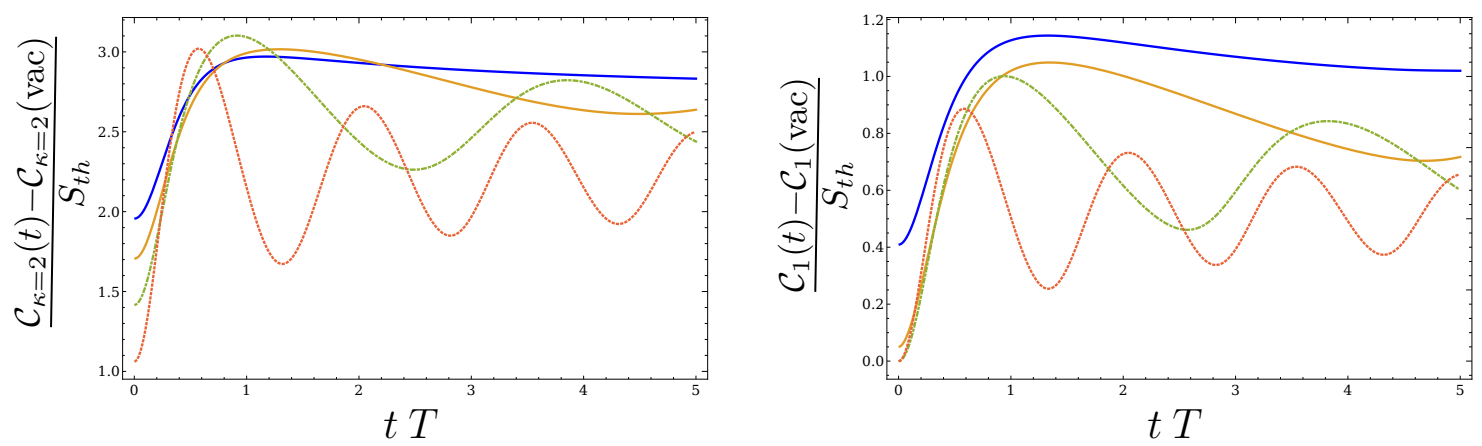

Figure 29. The time dependence of $\mathcal{C}_{\kappa=2}$ and $\mathcal{C}_{1}$ complexity of formation for cTFD state of a massive complex scalar theory in $d=1+1$ dimensions with $\tilde{Q}=1 / 10, \tilde{\gamma}=10$ and different masses. $\tilde{m}=1 / 5$ (blue), $\tilde{m}=1 / 2$ (orange), $\tilde{m}=1$ (dotted dashed green) and $\tilde{m}=2$ (dotted red). For large masses with respect to the thermal scale, there is an oscillatory behavior with period $\Delta t=\pi / \tilde{m}$. At late times, we observe a saturation to a constant value.
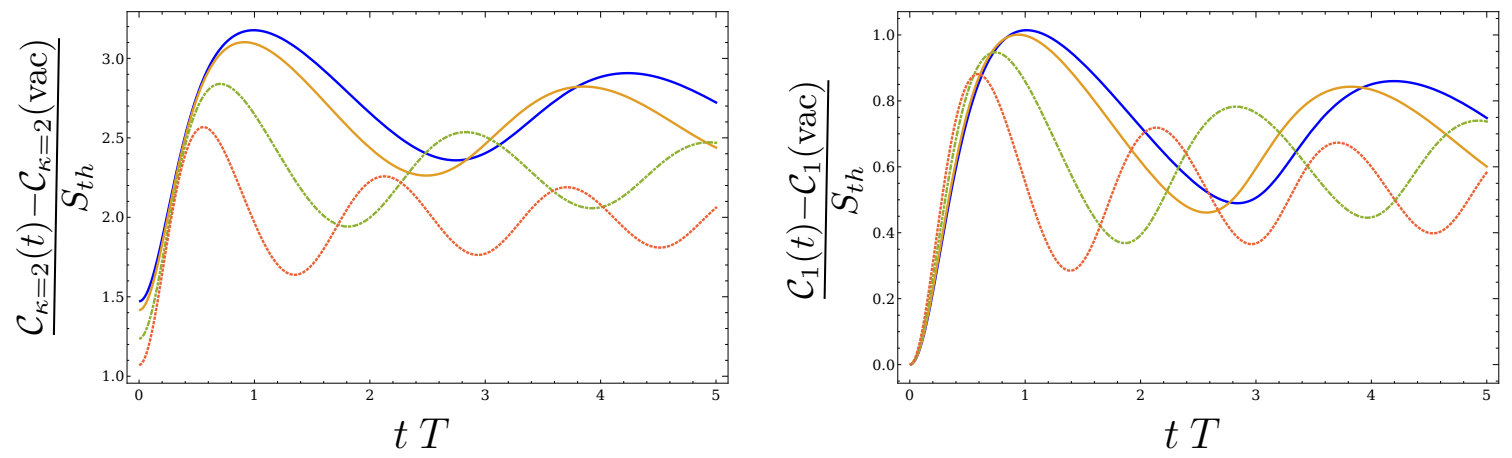

Figure 30. The time dependence of $\mathcal{C}_{\kappa=2}$ and $\mathcal{C}_{1}$ complexity of formation for cTFD state of a massive complex scalar theory in $d=1+1$ dimensions with $\tilde{m}=1, \tilde{\gamma}=10$ and different charges. $\tilde{Q}=10^{-6}$ (blue), $\tilde{Q}=10^{-1}$ (orange), $\tilde{Q}=1 / 2$ (dotted dashed green) and $\tilde{Q}=1$ (dotted red). For large charges with respect to the thermal scale, there is an oscillatory behavior with period $\Delta t=\pi \beta / \tilde{Q}$. At late times, we observe a saturation to a constant value.

the final expression for each of those parts is as following

$$
\begin{aligned}
I_{\mathrm{EH}}= & 2 \times \frac{V_{d-1}}{8 \pi G_{N}}\left(-\frac{2 d}{l^{2}}\right) \int_{r_{m}}^{r_{\infty}} d r r^{d-1}\left(r_{\infty}^{*}-r^{*}(r)\right) \\
I_{\text {Maxwell }}= & 2 \times \frac{V_{d-1}}{8 \pi G_{N}}(1-2 \gamma)\left(2(d-2) q^{2}\right) \int_{r_{m}}^{r_{\infty}} d r \frac{1}{r^{d-1}}\left(r_{\infty}^{*}-r^{*}(r)\right), \\
I_{\text {joint }}= & -2 \times \frac{V_{d-1}}{8 \pi G_{N}} r_{m}^{d-1} \log \left(\frac{l^{2}\left|f\left(r_{m}\right)\right|}{\tilde{\alpha}^{2} R^{2}}\right)-2 \times \frac{V_{d-1}}{8 \pi G} r_{\infty}^{d-1} \log \left(\frac{l^{2}\left|f\left(r_{\infty}\right)\right|}{\tilde{\alpha}^{2} R^{2}}\right), \\
I_{\mathrm{ct}}= & -2 \times \frac{V_{d-1}}{8 \pi G_{N}} r_{m}^{d-1}\left[\log \left(\frac{(d-1)^{2} l_{\mathrm{ct}}^{2} \tilde{\alpha}^{2} R^{2}}{r_{m}^{2} l^{2}}\right)+\frac{2}{d-1}\right] \\
& -2 \times \frac{V_{d-1}}{8 \pi G_{N}} r_{\infty}^{d-1}\left[\log \left(\frac{(d-1)^{2} l_{\mathrm{ct}}^{2} \tilde{\alpha}^{2} R^{2}}{r_{\infty}^{2} l^{2}}\right)+\frac{2}{d-1}\right],
\end{aligned}
$$



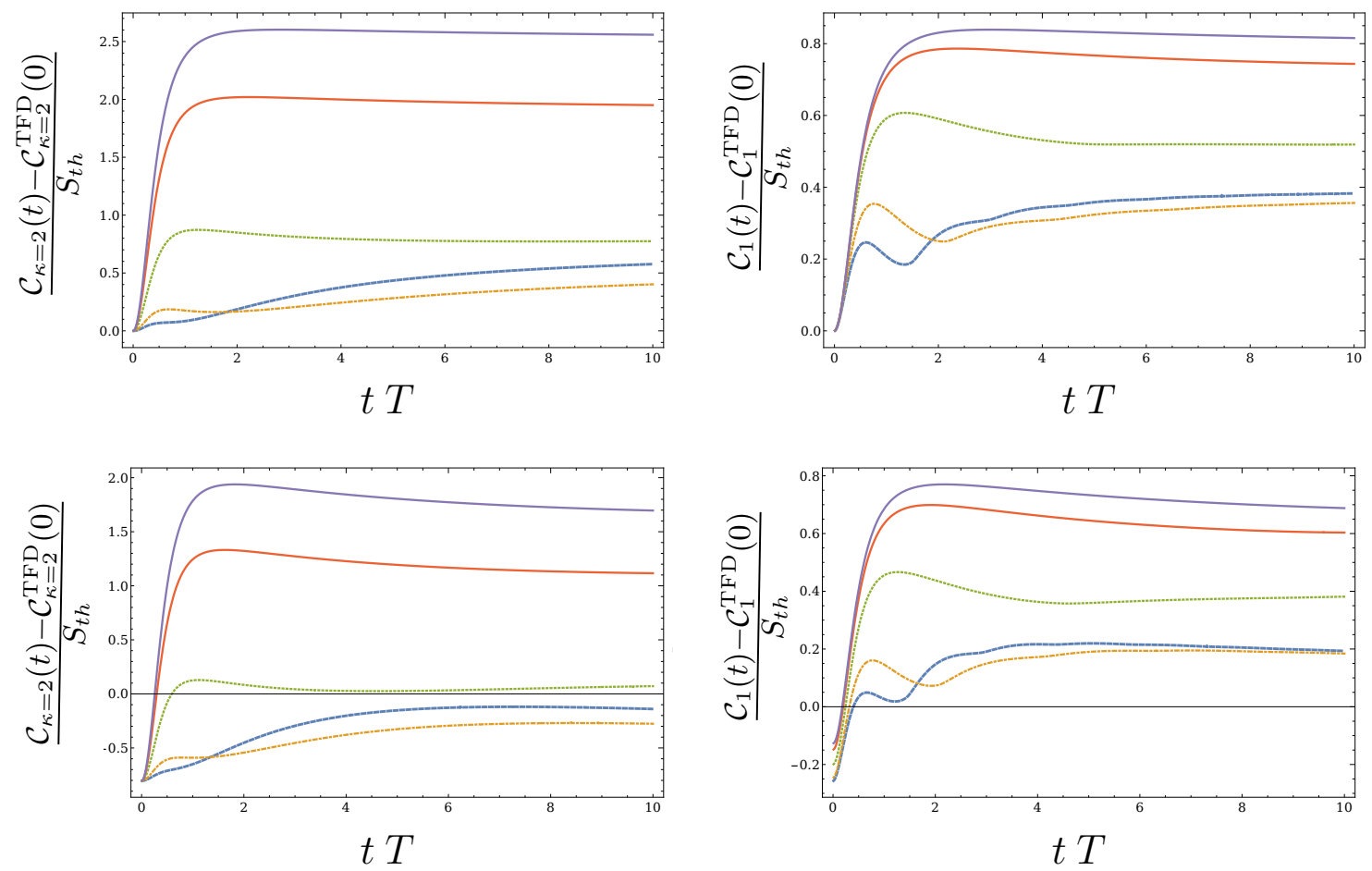

Figure 31. Up: The time dependence of $\mathcal{C}_{\kappa=2}$ and $\mathcal{C}_{1}$ complexities for TFD state of a massless real scalar theory in $d=1+1$ dimensions with $\tilde{\gamma}=10$ and different $\lambda_{R}$. Down: The time dependence of $\mathcal{C}_{\kappa=2}$ and $\mathcal{C}_{1}$ complexities for cTFD state of a massless complex scalar theory in $d=1+1$ dimensions with $\tilde{Q}=10^{-1}$ and $\tilde{\gamma}=10$ and different $\lambda_{R} \cdot \lambda_{R}=10$ (dashed blue), $\lambda_{R}=5$ (dotted dashed orange), $\lambda_{R}=1$ (dotted green), $\lambda_{R}=1 / 5$ (red) and $\lambda_{R}=1 / 10$ (purple).

where $r_{\infty}$ indicates the conformal boundary, $r_{m}$ is chosen for the joint points between two horizons, $\tilde{\alpha}$ refers to normalization of null boundaries of WDW patch ${ }^{6}$ and moreover total action is $I_{\mathrm{tot}}=I_{\mathrm{EH}}+I_{\text {Maxwee }}+I_{\text {joint }}+I_{\mathrm{ct}}$. To find the complexity of formation, we also need on-shell action for the vacuum state (pure AdS spacetime), $I^{\mathrm{vac}}=I_{\mathrm{Bulk}}^{\mathrm{vac}}+I_{\text {joint }}^{\mathrm{vac}}+I_{\mathrm{ct}}^{\mathrm{vac}}$, which its different parts are given by

$$
\begin{aligned}
I_{\mathrm{Bulk}}^{\mathrm{vac}} & =2 \times \frac{V_{d-1}}{8 \pi G_{N}}\left(-\frac{2 d}{l^{2}}\right) \int_{0}^{r_{\infty}} d r r^{d-1}\left(r_{\infty}^{* \mathrm{AdS}}-r^{* \mathrm{AdS}}(r)\right), \\
I_{\text {joint }}^{\mathrm{vac}} & =-2 \times \frac{V_{d-1}}{8 \pi G_{N}} r_{\infty}^{d-1} \log \left(\frac{l^{2}\left|f^{\mathrm{vac}}\left(r_{\infty}\right)\right|}{\tilde{\alpha}^{2} R^{2}}\right), \\
I_{\mathrm{ct}}^{\mathrm{vac}} & =-2 \times \frac{V_{d-1}}{8 \pi G_{N}} r_{\infty}^{d-1}\left[\log \left(\frac{4 l_{\mathrm{ct}}^{2} \tilde{\alpha}^{2} R^{2}}{r_{\infty}^{2} l^{2}}\right)+\frac{2}{d-1}\right] .
\end{aligned}
$$

It is easy to check that IR bulk divergences of total action in limit $r \rightarrow r_{\infty}$ are exactly the same as IR divergences for vacuum state. Therefore, holographic complexity of formation

$$
\Delta \mathcal{C}_{\mathrm{H}}=\frac{1}{\pi}\left(I_{\mathrm{tot}}-I^{\mathrm{vac}}\right)
$$

\footnotetext{
${ }^{6}$ One can choose different normalization for each null boundary of WDW patch but the final results do not alter.
} 


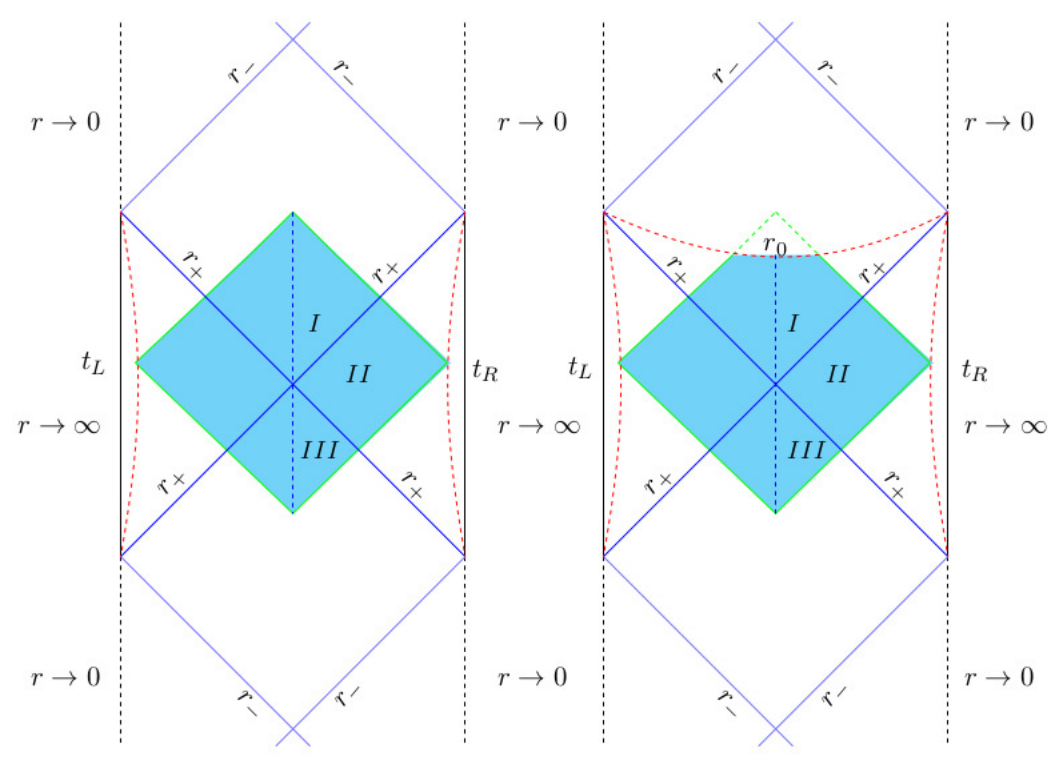

Figure 32. Left: Penrose diagram of charged AdS-BH and WDW patch is shown by shaded region. Right: Penrose diagram of charged AdS-BH and modified WDW patch is shown by shaded region. In comparison with left panel, one part of WDW patch is removed where $r_{-}<r_{0}<r_{+}$. Existence of this extra surface, $r=r_{0}$, implies that one also needs to consider GH term, reparametrization recovery counterterm together with two joint terms and bulk counterterm on that.

is finite and this cancellation of IR bulk divergences is consistent with the cancellation of UV divergences in the field theory side, (C.1). Apart from cancellation of IR divergences, there is another important reason for choosing AdS spacetime for reference state. It is shown [9] that the complexity of extremal BH diverges and therefore it can not be used as a meaningful tool for comparison. To compare with field theory results it will be useful to define following dimensionless parameters

$$
z=\frac{l}{r_{+}}, \quad y=\frac{r_{-}}{r_{+}}, \quad x=\frac{r}{r_{+}},
$$

which by using them for small charges we have ${ }^{7}$

$$
\begin{aligned}
R T & \sim \frac{r_{+}}{4 \pi L}\left(d-(d-2) y^{d-2}\right)+\mathcal{O}\left(y^{2(d-2)}\right), \\
\nu & \equiv \sqrt{\frac{C_{J}}{C_{T}}} \tilde{Q} \sim \frac{2 \sqrt{2} \pi(d-1)}{d \sqrt{d(d+1)}}\left[y^{\frac{d}{2}-1}+\left(\frac{3}{2}-\frac{2}{d}\right) y^{\frac{3 d}{2}-3}+\mathcal{O}\left(y^{\frac{3 d}{2}-1}\right)\right],
\end{aligned}
$$

where

$$
C_{J}=(d-2) \frac{\Gamma[d]}{2 \pi^{d / 2} \Gamma[d / 2]} \frac{l^{d-3}}{g^{2}}, \quad C_{T}=\frac{d+1}{d-1} \frac{\Gamma[d+1]}{8 \pi^{(d+2) / 2} \Gamma[d / 2]} \frac{l^{d-1}}{G_{N}} .
$$

The $C_{J}$ and $C_{T}$ are central charges associated with the two-point functions of the boundary currents and stress tensor

$$
\left\langle J_{\mu}(x) J_{\nu}(0)\right\rangle=\frac{C_{J}}{x^{2(d-1)}} \mathcal{A}_{\mu \nu}(x)+\ldots, \quad\left\langle T_{\mu \nu}(x) T_{\alpha \beta}(0)\right\rangle=\frac{C_{T}}{x^{2 d}} \mathcal{B}_{\mu \nu, \alpha \beta}+\ldots,
$$

\footnotetext{
${ }^{7}$ The 'non-normalizable' mode of the gauge potential is identified with $\tilde{\mu}$, i.e. $\tilde{\mu}=\lim _{r \rightarrow \text { bdy }} A_{t}$.
} 
with

$$
\mathcal{A}_{\mu \nu}=\eta_{\mu \nu}-2 \frac{x_{\mu} x_{\nu}}{x^{2}}, \quad \mathcal{B}_{\mu \nu, \alpha \beta}=\frac{1}{2}\left(\mathcal{A}_{\mu \nu}(x) \mathcal{A}_{\alpha \beta}(x)+\mathcal{A}_{\mu \beta}(x) \mathcal{A}_{\nu \alpha}(x)\right)-\frac{1}{d} \eta_{\mu \nu} \eta_{\alpha \beta} .
$$

Let us calculate the different contributions in (C.7) and (C.8) for $d=2+1$ and $d=3+1$ separately in the following two subsections.

\section{C.1 $d=2+1$ dimensions}

By defining

$$
\tilde{f}(x, y)=z^{2} f(r), \quad x^{*}(x, y)=r^{*}(r) / z^{2} r_{+},
$$

in $d=2+1$ dimensions we have

$$
\begin{aligned}
\tilde{f}(x, y)= & \frac{1}{x^{2}}(x-1)(x-y)\left(1+x+x^{2}+y+y^{2}+x y\right) \\
x^{*}(x, y)= & \frac{\left(3+6 y+10 y^{2}+6 y^{3}+3 y^{4}\right)}{\left(3+2 y+y^{2}\right)\left(1+2 y+3 y^{2}\right) \sqrt{3+2 y+3 y^{2}}} \arctan \left[\frac{1+2 x+y}{\sqrt{3+2 y+3 y^{2}}}\right] \\
& -\frac{1}{\left(-3+y+y^{2}+y^{3}\right)} \log |x-1|-\frac{y^{2}}{\left(1+y+y^{2}-3 y^{3}\right)} \log (x-y) \\
& -\frac{(1+y)^{3}}{2\left(3+2 y+y^{2}\right)\left(1+2 y+3 y^{2}\right)} \log \left(1+x+x^{2}+y+y^{2}+x y\right),
\end{aligned}
$$

where $r^{*}(r)=\int d r / f(r)$. According to (C.16) for boundary and joint surfaces one can see

$$
\begin{aligned}
& x_{\infty}^{*}=\frac{\pi\left(3+6 y+10 y^{2}+6 y^{3}+3 y^{4}\right)}{2\left(3+2 y+y^{2}\right)\left(1+2 y+3 y^{2}\right) \sqrt{3+2 y+3 y^{2}}}, \\
& x_{m} \simeq y\left(1+\exp \left(-\frac{\pi}{3 \sqrt{3} y^{2}}+\mathcal{O}\left(\frac{1}{y}\right)\right)\right),
\end{aligned}
$$

which they all together imply that

$$
\begin{aligned}
I_{\mathrm{AdS}} & =-\frac{V_{2} r_{+}^{2}}{4 G_{N}}\left(\frac{4 x_{\infty}^{2}}{\pi}+\frac{x_{\infty}^{2}}{\pi} \log \left(\frac{4 l_{\mathrm{ct}}^{2}}{l^{2}}\right)\right), \\
\mathrm{I}_{\mathrm{EH}} & =-\frac{V_{2} r_{+}^{2}}{4 G_{N}}\left(\frac{3 x_{\infty}^{2}}{\pi}+\frac{2 \sqrt{3}}{9}+\frac{2 y}{3 \sqrt{3}}+\frac{(9+4 \sqrt{3} \pi) y^{2}}{27 \pi}+\mathcal{O}\left(y^{3}\right)\right), \\
I_{\text {Maxwell }} & =\frac{V_{2} r_{+}^{2}}{4 G_{N}}(1-2 \gamma)\left(\frac{2}{3 \sqrt{3}}+\frac{2 y}{3 \sqrt{3}}+\left(\frac{4}{9 \sqrt{3}}+\frac{4}{3 \pi}\right) y^{2}+\mathcal{O}\left(y^{3}\right)\right), \\
I_{\text {joint }} & =\frac{V_{2} r_{+}^{2}}{4 G_{N}}\left(-\frac{x_{\infty}^{2}}{\pi} \log \left(\frac{l^{2} x_{\infty}^{2}}{R^{2} z^{2} \tilde{\alpha}^{2}}\right)+\frac{1}{3 \sqrt{3}}+\frac{y^{2}}{\pi} \log \left(\frac{R^{2} z^{2} \tilde{\alpha}^{2} y}{l^{2}}\right)\right), \\
I_{\mathrm{ct}} & =-\frac{V_{2} r_{+}^{2}}{4 G_{N}}\left(\frac{x_{\infty}^{2}}{\pi}+\frac{x_{\infty}^{2}}{\pi} \log \left(\frac{4 R^{2} z^{2} \tilde{\alpha}^{2} l_{\mathrm{ct}}^{2}}{x_{\infty}^{2} l^{4}}\right)+\frac{y^{2}}{\pi}+\frac{y^{2}}{\pi} \log \left(\frac{4 R^{2} z^{2} \tilde{\alpha}^{2} l_{c t}^{2}}{l^{4} y^{2}}\right)\right) .
\end{aligned}
$$

By using final expressions in (C.18) and noting to (C.9), (C.11) and (C.5), it is easy to see that

$$
\frac{\Delta \mathcal{C}_{\mathrm{H}}}{S_{\mathrm{th}}}=\frac{1}{3 \sqrt{3} \pi}+\frac{27}{64 \pi^{6}}\left(162 \log \left(\frac{3 \sqrt{3} l^{\frac{1}{3}}}{2^{\frac{11}{6}} l_{c t}^{\frac{1}{3}} \pi} \nu\right)\right) \nu^{4}+\mathcal{O}\left(\nu^{6}\right) .
$$




\section{C.2 $d=3+1$ dimensions}

In $d=3+1$ dimensions we have

$$
\begin{aligned}
\tilde{f}(x, y)= & \frac{\left(x^{2}-1\right)\left(x^{2}-y^{2}\right)\left(x^{2}+y^{2}+1\right)}{x^{4}}, \quad x_{m} \simeq y\left(1+\exp \left(-\frac{\pi}{2 y^{3}}+\mathcal{O}(1 / y)\right)\right), \\
x^{*}(x, y)= & \frac{\left(1+y^{2}\right)^{\frac{3}{2}}}{\left(2+y^{2}\right)\left(1+2 y^{2}\right)} \arctan \left(\frac{x}{\sqrt{1+y^{2}}}\right)+\frac{1}{2\left(1-y^{2}\right)\left(2+y^{2}\right)} \log \frac{|x-1|}{x+1} \\
& -\frac{y^{3}}{2\left(1-y^{2}\right)\left(1+2 y^{2}\right)} \log \frac{x-y}{x+y},
\end{aligned}
$$

which they all together imply that

$$
\begin{aligned}
I_{\mathrm{AdS}} & =-\frac{V_{3} r_{+}^{3}}{4 G_{N}}\left(\frac{10 x_{\infty}^{3}}{3 \pi}+\frac{x_{\infty}^{3}}{\pi} \log \left(\frac{9 l_{\mathrm{ct}}^{2}}{l^{2}}\right)\right), \\
I_{\mathrm{EH}} & =-\frac{V_{3} r_{+}^{3}}{4 G_{N}}\left(\frac{8 x_{\infty}^{3}}{3 \pi}+\frac{1}{2}+\frac{y^{2}}{2}+\mathcal{O}\left(y^{4}\right)\right), \\
I_{\text {Maxwell }} & =\frac{V_{3} r_{+}^{3}}{4 G_{N}}\left(\frac{1}{2}+\frac{y^{2}}{2}+\frac{2 y^{3}}{3 \pi}+\mathcal{O}\left(y^{4}\right)\right), \\
I_{\text {joint }} & =\frac{V_{3} r_{+}^{3}}{4 G_{N}}\left(-\frac{x_{\infty}^{3}}{\pi} \log \left(\frac{l^{2} x_{\infty}^{2}}{R^{2} z^{2} \tilde{\alpha}^{2}}\right)+\frac{1}{2}+\frac{y^{3}}{\pi} \log \left(\frac{R^{2} z^{2} \tilde{\alpha}^{2} y^{2}}{2 l^{2}}\right)\right), \\
I_{\mathrm{ct}} & =-\frac{V_{3} r_{+}^{3}}{4 G_{N}}\left(+\frac{2 x_{\infty}^{3}}{3 \pi}+\frac{x_{\infty}^{3}}{\pi} \log \left(\frac{9 R^{2} z^{2} \tilde{\alpha}^{2} l_{\mathrm{ct}}^{2}}{x_{\infty}^{2} l^{4}}\right)+\frac{2 y^{3}}{3 \pi}+\frac{y^{3}}{\pi} \log \left(\frac{9 R^{2} z^{2} \tilde{\alpha}^{2} l_{\mathrm{ct}}^{2}}{l^{4} y^{2}}\right)\right) .
\end{aligned}
$$

Having above results and noting to (C.9), (C.11) and (C.5) after short computation one can see

$$
\frac{\Delta \mathcal{C}_{\mathrm{H}}}{S_{\mathrm{th}}}=\frac{1}{2 \pi}+\frac{320 \sqrt{10}}{27 \pi^{5}}\left(\log \left(\frac{2^{\frac{5}{4}} \sqrt{5}}{3 \sqrt{3} \pi} \sqrt{\frac{l}{l_{\mathrm{ct}}}} \nu\right)\right) \nu^{3}+\mathcal{O}(\nu)^{4} .
$$

For the neutral case, $\tilde{Q}=0$, the complexity of formation is proportional to thermal entropy which is completely in agreement with the result of $\mathcal{C}_{1}$ complexity in LR basis. According to this agreement, it is claimed that [29] this norm of complexity is a corner stone of holographic complexity. Unlike the neutral case, QFT result (C.1) is independent of reference state scale $\omega_{R}$ but holographic results (C.19) and (C.22) depend to $l_{\text {ct }}$. Apart from that, in comparison with (C.1) also some terms in $\tilde{Q}$ expansion are missed in holographic results (C.19) and (C.22). Now we have at least four options. We can change the complexity in the QFT side by using the charged dependent penalty factors to set these extra terms zero or we can conclude that the CA proposal needs more boundary terms or we can say that these missing terms are actually zero in the strong coupling regime or we can conclude that $l_{\mathrm{ct}}$ is proportional to AdS radius $l$. If one chooses the first option, the new UV charged dependent divergences appear which they do not have the same counterpart in the holographic side because the on-shell action for the Maxwell term is finite and so the holographic UV divergent part is independent of chemical potential. According to the second option, recently two different boundary terms are proposed in [34] and [36]. In [34] 
a new boundary term for the Maxwell field is added to the action of Einstein-HilbertMaxwell theory (C.6) in $D=d+1=3+1$ dimensions $^{8}$

$$
I_{\tilde{\mu}}=\frac{\gamma}{g^{2}} \int_{\partial \mathcal{M}} d \Sigma_{\mu} F^{\mu \nu} A_{\nu}
$$

While introducing this boundary term does not change the equations of motion, it does change the nature of variational principle of the Maxwell field. That is, it changes the boundary conditions that must be imposed for consistency of the variational principle. If we use the Maxwell equations, $\nabla_{\mu} F^{\mu \nu}=0$, then this boundary term can be converted into a bulk term via stokes's theorem as

$$
I_{\tilde{\mu}, \text { on-shell }}=\frac{\gamma}{2 g^{2}} \int_{\mathcal{M}} d^{4} x \sqrt{-G} F_{\mu \nu} F^{\mu \nu} .
$$

According to (C.21), this new boundary term changes (C.22) to the following expression

$$
\frac{\Delta \mathcal{C}_{\mathrm{H}}}{S_{t h}}=\frac{(1-4 \gamma)}{3 \sqrt{3} \pi}-\frac{\sqrt{3} \gamma}{\pi^{2}}\left(\frac{G_{N}}{g^{2} l^{2}}\right) \tilde{Q}^{2}+\mathcal{O}\left(\tilde{Q}^{4}, l, l_{\mathrm{ct}}\right),
$$

where we have used the (C.11). For $\gamma=0$ and $\tilde{Q}=0$ we recover the known result [9]. In comparison with the QFT result (C.1), it is clear that adding boundary term (C.23) just recovers $\tilde{Q}^{2}$ term but $\tilde{Q}$ and $\tilde{Q}^{2} \log \tilde{Q}$ ones are remained absent. Apart from that, in the zero charge limit, the complexity of formation changes in comparison with neutral case, since this boundary term actually changes the boundary condition and therefore ensemble of system under consideration. Furthermore, by using this extra term, one can not see the dependence of complexity to the reference scale in general time, (4.49), since by comparison with QFT result at zero time we may choose $l_{\mathrm{ct}}$ as a function of the AdS scale $l$ and so no other arbitrary scale remains for another times.

Instead of changing the variational principle in [34, 35], authors [36, 37] suggest a very different understanding of the holographic complexity for JT gravity. This approach relies on defining a new cut-off surface, $r=r_{0}$, behind the outer horizon according to the left panel in figure 32. Based on this proposal, one needs to remove one part of the WDW patch and instead, add the following contributions to the action

$$
\begin{aligned}
I_{\mathrm{GH}} & =\frac{V_{d-1}}{8 \pi G_{N}} r_{0}^{d-1}\left(\partial_{r} f(r)+\frac{2(d-1)}{r} f(r)\right)\left(r^{*}\left(r_{0}\right)-r_{\infty}^{*}\right), \\
I_{\text {joint }} & =-\frac{V_{d-1}}{8 \pi G_{N}} r_{0}^{d-1} \log \left(\frac{l^{2}\left|f\left(r_{0}\right)\right|}{\tilde{\alpha}^{2} R^{2}}\right), \\
I_{\mathrm{ct}} & =-\frac{V_{d-1}}{8 \pi G_{N}} r_{0}^{d-1}\left[\log \left(\frac{(d-1)^{2} l_{\mathrm{ct}}^{2} \tilde{\alpha}^{2} R^{2}}{r_{0}^{2} l^{2}}\right)+\frac{2}{d-1}\right] . \\
I_{\mathrm{ct}}^{\text {new }} & =\frac{V_{d-1}}{8 \pi G} r_{0}^{d-1}\left(\frac{2(d-1)}{l} \sqrt{f\left(r_{0}\right)}\left(r_{\infty}^{*}-r^{*}\left(r_{0}\right)\right)\right),
\end{aligned}
$$

where $I_{\mathrm{ct}}^{\text {new }}$ is standard volume counterterm which is calculated on the $r=r_{0}$ hypersurface. By choosing the behind the horizon cut-off $r_{0}$ as following

$$
x_{0} \equiv \frac{r_{0}}{r_{+}}=y^{\frac{(d-2)}{2}}\left(1+\exp \left[-\frac{c_{3}}{y^{3}}-\frac{c_{2}}{y^{2}}-\frac{c_{1}}{y}+c_{0}+\tilde{c}_{1} y+\ldots\right]\right),
$$

\footnotetext{
${ }^{8}$ Adding this term puts the electric and magnetic charges on an equal footing in the holographic complexity.
} 
for $d=2+1$ we have

$$
\begin{aligned}
I_{\mathrm{EH}}^{r_{0}} & =\frac{V_{2} r_{+}^{2}}{4 G_{N}}\left(-\frac{3 x_{\infty}^{2}}{2 \pi}-\frac{1}{3 \sqrt{3}}-\frac{y}{3 \sqrt{3}}\right)+\mathcal{O}\left(y^{\frac{3}{2}}\right), \\
I_{\text {Maxwell }}^{r_{0}} & =\frac{V_{2} r_{+}^{2}}{4 G_{N}}\left(\frac{(1-2 \gamma) y^{\frac{1}{2}}}{3 \sqrt{3}}+\frac{(1-2 \gamma) y}{3 \sqrt{3}}\right)+\mathcal{O}\left(y^{\frac{3}{2}}\right), \\
I_{\mathrm{GH}}^{r_{0}} & =\frac{V_{2} r_{+}^{2}}{4 G_{N}}\left(\frac{1}{2 \sqrt{3}}-\frac{y^{\frac{1}{2}}}{3 \sqrt{3}}+\frac{3 y}{4 \pi}\right)+\mathcal{O}\left(y^{\frac{3}{2}}\right), \\
I_{\text {joint }}^{r_{0}} & =\frac{V_{2} r_{+}^{2}}{4 G_{N}}\left(\frac{y}{4 \pi} \log \left(\frac{z^{4} R^{4} \tilde{\alpha}^{4} y}{l^{4}}\right)\right)+\mathcal{O}\left(y^{\frac{3}{2}}\right), \\
I_{\mathrm{ct}}^{r_{0}} & =-\frac{V_{2} r_{+}^{2}}{4 G_{N}} \frac{y}{2 \pi}\left(1-\log \left(\frac{y l^{4}}{4 z^{2} l_{\mathrm{ct}}^{2} R^{2} \tilde{\alpha}^{2}}\right)\right), \\
I_{\mathrm{ct}}^{\text {new }} & \sim \mathcal{O}\left(y^{\frac{3}{4}}\right) .
\end{aligned}
$$

Also for $d=3+1$ one can check that

$$
\begin{aligned}
I_{\mathrm{EH}}^{r_{0}} & =\frac{V_{3} r_{+}^{3}}{4 G_{N}}\left(-\frac{4 x_{\infty}^{3}}{3 \pi}-\frac{1}{4}+\frac{y}{4 \pi}-\frac{1+2 \pi}{8 \pi} y^{2}+\frac{5 y^{3}}{24 \pi}\right)+\mathcal{O}\left(y^{4}\right), \\
I_{\text {Maxwell }}^{r_{0}} & =\frac{V_{3} r_{+}^{3}}{4 G_{N}}\left(\frac{1}{4}-\frac{y}{4 \pi}+\frac{(1+2 \pi)}{8 \pi} y^{2}+\frac{y^{3}}{8 \pi}\right)+\mathcal{O}\left(y^{4}\right), \\
I_{\mathrm{GH}}^{r_{0}} & =\frac{V_{3} r_{+}^{3}}{4 G_{N}}\left(\frac{\left(\pi-2 c_{3}\right)}{4 \pi}-\frac{\left(1+2 c_{2}\right)}{4 \pi} y-\frac{\left(-1+4 c_{1}\right)}{8 \pi} y^{2}-\frac{\left(-7-4 c_{0}+4 \log 2\right)}{8 \pi} y^{3}\right)+\mathcal{O}\left(y^{4}\right), \\
I_{\text {joint }}^{r_{0}} & =\frac{V_{3} r_{+}^{3}}{4 G_{N}}\left(\frac{c_{3}}{2 \pi}+\frac{c_{2}}{2 \pi} y+\frac{c_{1}}{2 \pi} y^{2}+\frac{1}{2 \pi}\left(-c_{0}+2 \log \left[\frac{z R y \tilde{\alpha}}{\sqrt{2} l}\right]\right) y^{3}\right), \\
I_{\mathrm{ct}}^{r_{0}} & =-\frac{V_{3} r_{+}^{3}}{4 G_{N}} \frac{y^{3}}{2 \pi}\left(\frac{2}{3}-2 \log \left[\frac{l^{2} y}{3 z l_{\mathrm{ct}} R \tilde{\alpha}}\right]\right), \quad I_{\mathrm{ct}}^{\text {new }}=0 .
\end{aligned}
$$

It is also easy to see that by choosing the behind horizon cut off according to (C.27) (for small charges), not only the complexity of formation smoothly approach to the nuetral case in the limit $y \rightarrow 0$ but also the complexity growth rate matches with the Lloyd's bound in the late time in the same limit. Putting all ingredients (C.28) and (C.29) together the complexity for formation for $d=2+1$ and $d=3+1$ respectively becomes

$$
\begin{aligned}
\frac{\Delta \mathcal{C}_{\text {holo }}}{S_{\text {th }}}= & \frac{(1-2 \gamma)}{3 \sqrt{3} \pi}-\left(\frac{\gamma}{\sqrt{3} \pi^{\frac{3}{2}}} \frac{G_{N}^{\frac{1}{2}}}{g l}\right) \tilde{Q} \\
& +\frac{G_{N}}{16 \pi^{3} g^{2} l^{2}}\left(9-16 \sqrt{3} \pi \gamma-54 \log \left(\frac{2^{\frac{5}{3}} \pi^{\frac{1}{2}}}{3} \frac{g l^{\frac{1}{3}} l_{\mathrm{ct}}^{\frac{2}{3}}}{G_{N}^{\frac{1}{2}}}\right)+54 \log \tilde{Q}\right) \tilde{Q}^{2}+\mathcal{O}\left(\tilde{Q}^{3}\right),
\end{aligned}
$$

and

$$
\begin{aligned}
\frac{\Delta \mathcal{C}_{\text {holo }}}{S_{\mathrm{th}}}= & \frac{1}{2 \pi}-\left(\frac{1}{\sqrt{3} \pi^{\frac{5}{2}}} \frac{G_{N}^{\frac{1}{2}}}{g l}\right) \tilde{Q}+\left(\frac{2 G_{N}}{3 \pi^{3} g^{2} l^{2}}\right) \tilde{Q}^{2}+ \\
& +\frac{8}{3 \sqrt{3} \pi^{\frac{7}{2}}}\left(\frac{G_{N}}{g^{2} l^{2}}\right)^{\frac{3}{2}}\left(7-16 \log \left(\frac{9 \pi}{2^{\frac{13}{4}}} \frac{g^{2} l l_{\mathrm{ct}}}{G_{N}}\right)+32 \log \tilde{Q}\right) \tilde{Q}^{3}+\mathcal{O}\left(\tilde{Q}^{4}\right) .
\end{aligned}
$$


Intriguingly, holographic results (C.30)-(C.31) which are obtained in presence of behind the horizon cut-off are compatible with field theory result (C.1) since we could recover all the $\tilde{Q}$ terms. Moreover, the QFT result (C.1) does not depend on the reference scale and its consistency with holographic results (C.30)-(C.31) implies that $l_{\mathrm{ct}}$ is actually proportional to AdS scale. It is worth noting that the identification between $l_{\text {ct }}$ and $l$ implies that the complexity of formation as a function of $G_{N}, g, l$ can be completely expressed as a ratio of two boundary central charges, $C_{J} / C_{T} \cdot{ }^{9}$ Moreover, at finite chemical potential and zero temperature $\tilde{Q} \equiv \tilde{\mu} / T \rightarrow \infty$, the complexity of formation of holographic states (C.30)(C.31) diverges similarly to the complexity of formation of free field theory (C.1). According to the above analysis, we see that the $F_{1}$ complexity (4.35) not only is compatible with holographic complexity for neutral $\mathrm{BH}$ but also works for $\mathrm{U}(1)$ electric charged ones.

To close this section let us emphasize that we have chosen an arbitrary length scale $l_{\mathrm{ct}} \sim l$ by comparing with the QFT results for complexity of formation. This identification is obtained for zero time $t_{L}=t_{R}=0$ and for another times, apart from geometrical variables $G_{N}, l, g$ we also have the time $\tau=t_{L}+t_{R}$ itself. This extra dimensionful variable may also appear in the expression of length scale $l_{\text {ct }}$. This time dependency of $l_{\text {ct }}$ can cause the complexity itself becomes a function of a completely new scale, similar to the appearance of $\omega_{R}$ in non-zero times in QFT side, (4.49). But this is not a good way to reconstruct the dependence on $\omega_{R}$ for nonzero times because of two reasons. Firstly, the time dependency of $l_{\text {ct }}$ causes the counterterterm action, $I_{\text {ct }}$, breaks bulk general covariance. Secondly, this time dependency implies that UV divergences of holographic complexity for AdS geometry become time-dependent which by comparison with (C.8) it is clear that this can not be correct. This observation needs to be considered more carefully and we hope to address a resolution for that in near future.

Open Access. This article is distributed under the terms of the Creative Commons Attribution License (CC-BY 4.0), which permits any use, distribution and reproduction in any medium, provided the original author(s) and source are credited.

\section{References}

[1] S. Ryu and T. Takayanagi, Holographic derivation of entanglement entropy from AdS/CFT, Phys. Rev. Lett. 96 (2006) 181602 [hep-th/0603001] [INSPIRE].

[2] S. Ryu and T. Takayanagi, Aspects of Holographic Entanglement Entropy, JHEP 08 (2006) 045 [hep-th/0605073] [INSPIRE].

[3] B. Swingle, Entanglement Renormalization and Holography, Phys. Rev. D 86 (2012) 065007 [arXiv: 0905.1317] [INSPIRE].

[4] L. Susskind, Entanglement is not enough, Fortsch. Phys. 64 (2016) 49 [arXiv:1411.0690] [INSPIRE].

\footnotetext{
${ }^{9}$ Another choice can be $l_{\mathrm{ct}} \sim l \times \mathbf{L} / \delta$ which implies that the leading divergence of complexity remains as $1 / \delta^{d-1} \log \delta$. But this choice implies that the complexity of formation in (C.30)-(C.31) not only depends on the arbitrary length scale $\mathbf{L}$ but also it becomes UV divergent which is clearly in contradiction with the QFT result (C.1).
} 
[5] L. Susskind, Computational Complexity and Black Hole Horizons, Fortsch. Phys. 64 (2016) 24 [Addendum ibid. 64 (2016) 44] [arXiv:1403.5695] [INSPIRE].

[6] A.R. Brown, D.A. Roberts, L. Susskind, B. Swingle and Y. Zhao, Holographic Complexity Equals Bulk Action?, Phys. Rev. Lett. 116 (2016) 191301 [arXiv:1509.07876] [INSPIRE].

[7] A.R. Brown, D.A. Roberts, L. Susskind, B. Swingle and Y. Zhao, Complexity, action and black holes, Phys. Rev. D 93 (2016) 086006 [arXiv:1512.04993] [InSPIRE].

[8] S. Lloyd, Ultimate Physical limits to computation, Nature 406 (2000) 1047 [quant-ph/9908043].

[9] D. Carmi, S. Chapman, H. Marrochio, R.C. Myers and S. Sugishita, On the Time Dependence of Holographic Complexity, JHEP 11 (2017) 188 [arXiv:1709.10184] [InSPIRE].

[10] D. Carmi, R.C. Myers and P. Rath, Comments on Holographic Complexity, JHEP 03 (2017) 118 [arXiv: 1612.00433] [INSPIRE].

[11] S.H. Shenker and D. Stanford, Black holes and the butterfly effect, JHEP 03 (2014) 067 [arXiv: 1306.0622] [INSPIRE].

[12] J. Maldacena and L. Susskind, Cool horizons for entangled black holes, Fortsch. Phys. 61 (2013) 781 [arXiv:1306.0533] [INSPIRE].

[13] A. Almheiri, D. Marolf, J. Polchinski, D. Stanford and J. Sully, An Apologia for Firewalls, JHEP 09 (2013) 018 [arXiv: 1304.6483] [INSPIRE].

[14] K. Papadodimas and S. Raju, An Infalling Observer in AdS/CFT, JHEP 10 (2013) 212 [arXiv:1211.6767] [INSPIRE].

[15] R. Jefferson and R.C. Myers, Circuit complexity in quantum field theory, JHEP 10 (2017) 107 [arXiv: 1707.08570] [INSPIRE].

[16] M.A. Nielsen, A geometric approach to quantum circuit lower bounds, quant-ph/0502070.

[17] M.A. Nielsen, M.R. Dowling, M. Gu and A.M. Doherty, Quantum Computation as Geometry, Science 311 (2006) 1133 [quant-ph/0603161].

[18] M.A. Nielsen and M.R. Dowling, The geometry of quantum computation, quant-ph/0701004.

[19] M. Guo, J. Hernandez, R.C. Myers and S.-M. Ruan, Circuit Complexity for Coherent States, JHEP 10 (2018) 011 [arXiv: 1807.07677] [INSPIRE].

[20] L. Hackl and R.C. Myers, Circuit complexity for free fermions, JHEP 07 (2018) 139 [arXiv: 1803.10638] [INSPIRE].

[21] E. Caceres, S. Chapman, J.D. Couch, J.P. Hernandez, R.C. Myers and S.-M. Ruan, Complexity of Mixed States in QFT and Holography, arXiv:1909.10557 [INSPIRE].

[22] J. Jiang and X. Liu, Circuit Complexity for Fermionic Thermofield Double states, Phys. Rev. D 99 (2019) 026011 [arXiv: 1812.00193] [INSPIRE].

[23] J. Jiang, J. Shan and J. Yang, Circuit complexity for free Fermion with a mass quench, arXiv: 1810.00537 [INSPIRE].

[24] R. Khan, C. Krishnan and S. Sharma, Circuit Complexity in Fermionic Field Theory, Phys. Rev. D 98 (2018) 126001 [arXiv:1801.07620] [INSPIRE].

[25] A. Bhattacharyya, A. Shekar and A. Sinha, Circuit complexity in interacting QFTs and RG flows, JHEP 10 (2018) 140 [arXiv: 1808.03105] [INSPIRE]. 
[26] P. Caputa, N. Kundu, M. Miyaji, T. Takayanagi and K. Watanabe, Anti-de Sitter Space from Optimization of Path Integrals in Conformal Field Theories, Phys. Rev. Lett. 119 (2017) 071602 [arXiv: 1703.00456] [INSPIRE].

[27] P. Caputa, N. Kundu, M. Miyaji, T. Takayanagi and K. Watanabe, Liouville Action as Path-Integral Complexity: From Continuous Tensor Networks to AdS/CFT, JHEP 11 (2017) 097 [arXiv: 1706. 07056] [INSPIRE].

[28] H.A. Camargo, M.P. Heller, R. Jefferson and J. Knaute, Path integral optimization as circuit complexity, Phys. Rev. Lett. 123 (2019) 011601 [arXiv: 1904.02713] [INSPIRE].

[29] S. Chapman et al., Complexity and entanglement for thermofield double states, SciPost Phys. 6 (2019) 034 [arXiv: 1810.05151] [INSPIRE].

[30] S. Chapman and H.Z. Chen, Complexity for Charged Thermofield Double States, arXiv:1910.07508 [INSPIRE].

[31] A.B. Klimov and S.M. Chumakov, A group-theoretical approach to quantum optics: models of atom-field interactions, Wiley-VCH, (2009).

[32] L. Lehner, R.C. Myers, E. Poisson and R.D. Sorkin, Gravitational action with null boundaries, Phys. Rev. D 94 (2016) 084046 [arXiv: 1609.00207] [InSPIRE].

[33] M. Sinamuli and R.B. Mann, Holographic Complexity and Charged Scalar Fields, Phys. Rev. D 99 (2019) 106013 [arXiv: 1902.01912] [INSPIRE].

[34] A.R. Brown, H. Gharibyan, H.W. Lin, L. Susskind, L. Thorlacius and Y. Zhao, Complexity of Jackiw-Teitelboim gravity, Phys. Rev. D 99 (2019) 046016 [arXiv:1810.08741] [InSPIRE].

[35] K. Goto, H. Marrochio, R.C. Myers, L. Queimada and B. Yoshida, Holographic Complexity Equals Which Action?, JHEP 02 (2019) 160 [arXiv:1901.00014] [INSPIRE].

[36] A. Akhavan, M. Alishahiha, A. Naseh and H. Zolfi, Complexity and Behind the Horizon Cut Off, JHEP 12 (2018) 090 [arXiv:1810.12015] [INSPIRE].

[37] M. Alishahiha, On complexity of Jackiw-Teitelboim gravity, Eur. Phys. J. C 79 (2019) 365 [arXiv: 1811.09028] [INSPIRE]. 